\title{
VARIÉTÉS SPHÉRIQUES DE TYPE A
}

\author{
par D. LUNA
}

\section{Introduction}

Soit $\mathrm{G}$ un groupe algébrique réductif connexe (le corps de base $k$ étant algébriquement clos et de caractéristique nulle). Soient B un sous-groupe de Borel de $\mathrm{G}$, et $\mathrm{T}$ un tore maximal de $\mathrm{B}$.

0.1 Une G-variété algébrique $\mathrm{X}$ est appelée sphérique si elle est normale et si $\mathrm{B}$ a une orbite dense dans $\mathrm{X}$. Un sous-groupe algébrique $\mathrm{H}$ de $\mathrm{G}$ est dit sphérique si l'espace homogène $\mathrm{G} / \mathrm{H}$ est sphérique.

Si $\mathrm{G}=\mathrm{B}=\mathrm{T}$ est un tore, les variétés sphériques ne sont rien d'autre que les variétés toriques (i.e. les $\mathrm{T}$-variétés algébriques normales dans lesquelles $\mathrm{T}$ a une orbite dense). Il est bien connu que ces dernières se laissent décrire de façon combinatoire (voir par exemple $[\mathrm{Fu}]$ ). Rappelons brièvement comment. Notons $\Xi(\mathrm{T})$ le groupe de caractères de $\mathrm{T}$. Soit $\mathrm{X}$ une $\mathrm{T}$-variété torique. Un premier invariant associé à $\mathrm{X}$ est le sous-groupe $\Xi_{\mathrm{X}}$ de $\Xi(\mathrm{T})$ formé des poids de $\mathrm{T}$ dans $k(\mathrm{X})$ (le corps des fonctions rationnelles sur $\mathrm{X}$ ). Cet invariant détermine l'orbite dense de $\mathrm{T}$ dans $\mathrm{X}$. L'invariant plus fin qui détermine $\mathrm{X}$ est un certain objet combinatoire appelé «éventail», qui «vit» dans le $\mathbf{Q}$-espace vectoriel $N_{X}=\operatorname{Hom}_{\mathbf{Z}}\left(\Xi_{X}, \mathbf{Q}\right)$. Tout sous-groupe $\Xi$ de $\Xi(T)$, et tout éventail dans $\mathrm{N}=\operatorname{Hom}_{\mathbf{z}}(\Xi, \mathbf{Q})$ provient d'une (unique) T-variété torique.

Il est naturel de vouloir généraliser ce qui précède aux variétés sphériques. C'est ce qu'on accomplira dans ce travail, dans le cas où le groupe adjoint de $\mathrm{G}$ est de type A, c'est-à-dire est un produit de groupes PGL.

Voici un premier aperçu de nos résultats (pour les énoncés précis, voir §2). Le groupe $G$ est bien sûr déterminé par son système des racines et le réseau $\Xi(B)$. Un premier invariant associé à une $\mathrm{G}$-variété sphérique $\mathrm{X}$ est ici le sous-groupe $\Xi_{\mathrm{X}}$ de $\Xi(\mathrm{B})$, formé des poids de $\mathrm{B}$ dans $k(\mathrm{X})$. Mais dans le contexte sphérique, cet invariant ne caractérise plus l'orbite dense $\mathrm{X}_{\mathrm{G}}^{\circ}$ de $\mathrm{G}$ dans $\mathrm{X}$. Quelle «donnée sphérique» faut-il adjoindre à $\Xi_{\mathrm{X}}$ pour déterminer $\mathrm{X}_{\mathrm{G}}^{\circ}$ ? Le principal objectif de ce travail consiste à répondre à cette question (voir §2). La théorie des plongements des espaces homogènes sphériques ([Knl], [Br6]) fournit alors les invariants plus fins déterminant $\mathrm{X}$, les «éventails coloriés», qui vivent dans le Q-espace vectoriel «colorié» $\mathrm{N}_{\mathrm{X}}=\operatorname{Hom}_{\mathbf{Z}}\left(\Xi_{\mathrm{X}}, \mathbf{Q}\right)$. Ici aussi, tout sous-groupe $\Xi$ de $\Xi(\mathrm{B})$ muni d'une donnée sphérique, et tout éventail colorié dans $\mathrm{N}=\operatorname{Hom}_{\mathbf{z}}(\Xi, \mathbf{Q})$ provient d'une (unique) Gvariété sphérique. 
0.2 Pourquoi s’intéresser aux variétés sphériques? Voici quelques éléments de réponse.

Une G-variété affine (normale) est sphérique si et seulement si $k[\mathrm{X}]$, l'algèbre des fonctions régulières sur $\mathrm{X}$, est un G-module (rationnel) sans multiplicité. Ces variétés jouent par exemple un rôle important comme principe organisationnel dans la théorie des invariants des groupes classiques (voir [Vul] et [Ho]).

Un autre endroit où l'on rencontre les variétés sphériques est la théorie des opérations hamiltoniennes. Lorsque $k=\mathrm{C}$, à toute $\mathrm{G}$-variété algébrique projective lisse polarisée (i.e. munie d'un fibré en droites ample G-linéarisé, on peut associer une opération hamiltonienne de $\mathrm{K}$ (le groupe compact maximal de $\mathrm{G}$ ); de plus, cette opération hamiltonienne est «sans multiplicité» (au sens de [G-S], voir aussi $[\mathrm{H}-\mathrm{W}]$ ), si et seulement si la G-variété algébrique est sphérique ([Br3]). Les variétés sphériques fournissent donc des exemples (nombreux) d'opérations hamiltoniennes sans multiplicité (les géomètres différentiels voient bien sûr tout à l'envers : ils considèrent les premières comme «quantisations géométriques complexes» des secondes, voir par exemple [Wo]). Le problème (encore ouvert) de la classification des opérations hamiltoniennes sans multiplicité est très lié à celui des variétés sphériques affines lisses (voir [Kn3] et $[\mathrm{Kn} 4])$.

Parmi les exemples les plus connus de variétés sphériques, mentionnons les espaces homogènes symétriques et leurs «complétions» (voir [DeC-P]). Pour des renseignements supplémentaires, voir aussi [Br4], [Br5] et [Br7].

0.3 Expliquons maintenant comment est organisé ce travail.

Le $\S 1$ contient divers rappels concernant les variétés sphériques. On y rappelle en particulier la notion de «variété magnifique» qui joue un rôle central dans la théorie (un peu comme les variétés de drapeaux dans la théorie des groupes semi-simples).

Au $\S 2$ seront énoncés les résultats principaux :

a) la classification des variétés magnifiques (de type $\mathrm{A}$ ), en termes de «systèmes sphériques» (théorème 1);

b) celle des espaces homogènes sphériques, en termes de «données sphériques homogènes » (théorème 2);

c) enfin, celle de toutes les variétés sphériques (théorème 3, qui découle immédiatement du théorème 2 et de la théorie des plongements des espaces homogènes sphériques).

Les $\S 3$ et 4 contiennent des préparatifs à la preuve du théorème 1 (qui, elle, fera l'objet du §5). Au §3 on présentera diverses constructions concernant les variétés magnifiques, et leurs analogues combinatoires pour les systèmes sphériques : «localisation», «quotient», «induction parabolique», «décomposition en produit fibré» et «fibration projective». Le $\S 4$ concerne la structure des systèmes sphériques de type A : on y explicitera 29 systèmes sphériques «primitifs» (dont certains sont des 
familles dépendant d'un ou de deux paramètres entiers, et d'autres sont des cas isolés), à partir desquels tout système de type $\mathrm{A}$ s'obtient par induction parabolique, produit fibré, et fibration projective. Au $\S 5$, on vérifie d'abord le théorème 1 pour les systèmes primitifs, puis grâce aux résultats des $\S 3$ et 4 , on en déduit le cas général.

$\mathrm{Au} \S 6$ on introduira une «clôture sphérique» pour tout sous-groupe sphérique, puis on établira quelques propriétés de cette notion, qui permettront au $\$ 7$ de réduire le théorème 2 au théorème 1 . Les $§ 6-7$ sont (presque) indépendants des §3-5.

0.4 En matière de classification (à part quelques cas isolés) seuls étaient connus jusqu'ici les sous-groupes sphériques réductifs ([Mi], [Br2]), et les variétés magnifiques de rang 1 ([Ak], $[\mathrm{Brl}]$ et $2([\mathrm{Wa}])\left({ }^{1}\right)$; résultats qui étaient présentés sous forme de tables, «en vrac» et sans ordre apparent. Le présent travail non seulement se veut plus complet mais aussi plus systématique. On y ramène la classification de toutes les variétés magnifiques (et de tous les sous-groupes sphériques) à des objets combinatoires qui ne dépendent que du système des racines (pour les systèmes sphériques, on propose aussi une présentation sous forme de «diagrammes», partant du diagramme de Dynkin, voir $\S 4)$.

Bien que dans ce travail le théorème 1 ne soit prouvé que lorsque le groupe est de type $\mathrm{A}$, on a pris soin de formuler tous les énoncés pour un groupe semi-simple (connexe) quelconque, et une partie de la preuve est conduite dans ce cadre général (seuls le $\S 3, n^{\circ} 6$, le $\S 4$ et le $\S 5$ à partir du $n^{\circ} 2$ font appel à l'hypothèse restrictive). Les théorèmes 2 et 3 seront établis pour tout groupe réductif dont le groupe adjoint vérifie le théorème 1 .

0.5 Terminons cette introduction par quelques considérations plus spéculatives. Il y a manifestement des analogies entre la théorie des groupes réductifs et celle des espaces homogènes sphériques. Par exemple, la structure des groupes réductifs se réduit à celle des groupes semi-simples, un peu comme la structure des espaces homogènes sphériques se ramène à celle des variétés magnifiques (voir §6 et 7); à toute variété sphérique, comme à tout groupe réductif, on peut associer un «groupe de Weyl» (voir [Kn5]), etc.

Il est bien connu que chaque groupe semi-simple peut être présenté comme amalgame de certains de ses sous-groupes semi-simples de rang 2. De même, au niveau combinatoire, chaque système sphérique est visiblement un «amalgame» de ses «sous-systèmes» de rang 2. Peut-on transposer cette idée dans le contexte géométrique des variétés magnifiques?

${ }^{1}$ ) Le rang d'une variété sphérique $\mathrm{X}$ est par définition celui du groupe abélien libre $\Xi_{\mathrm{X}}$ défini ci-dessus. 
TABLE DES MATIÈRES

$\S 1$ Rappels sur les variétés sphériques . . . . . . . . . . . . . . . . . . . . . . . . . . . . . . . . 164

1.1 Espaces vectoriels coloriés . . . . . . . . . . . . . . . . . . . . . . . . . . . . . . . 165

1.2 Racines sphériques et variétés magnifiques de rang 1 . . . . . . . . . . . . . . . . . . . 165

1.3 Variétés magnifiques de rang quelconque . . . . . . . . . . . . . . . . . . . . . . . . . . 167

1.4 Relations entre couleurs et racines sphériques . . . . . . . . . . . . . . . . . . . . . . . . . . . . . . . . . . . . . 167

\$2 Énoncé des résultats principaux . . . . . . . . . . . . . . . . . . . . . . . . . . . . . . . . . . . . . . . . . . . . . . 168

$\S 3$ Variétés magnifiques et systèmes sphériques . . . . . . . . . . . . . . . . . . . . . . . . . 171

3.1 Sous-systèmes . . . . . . . . . . . . . . . . . . . . . . . . . . . . . . . . . . . . 171

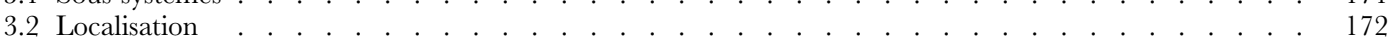

3.3 Sous-ensembles distingués de couleurs et quotients . . . . . . . . . . . . . . . . . . . . . 173

3.4 Induction parabolique . . . . . . . . . . . . . . . . . . . . . . . . . . . . . . . . . 175

3.5 Produits fibrés . . . . . . . . . . . . . . . . . . . . . . . . . . . . . . . . . . . . 177

3.6 Fibrations projectives . . . . . . . . . . . . . . . . . . . . . . . . . . . . . . . . . . . 179

§4 Étude combinatoire des systèmes sphériques de type A . . . . . . . . . . . . . . . . . . . . . . . 183

4.1 Diagrammes . . . . . . . . . . . . . . . . . . . . . . . . . . . . . . . . . . . . . 183

4.2 La liste des cas «primitifs» . . . . . . . . . . . . . . . . . . . . . . . . . . . . . 187

4.3 La $\Delta$-connexité . . . . . . . . . . . . . . . . . . . . . . . . . . . . . . . . . . . . . . . . . . . . . . . . 192

4.4 Composantes $\Delta$-connexes effaçables . . . . . . . . . . . . . . . . . . . . . . . . . . . . . . . . . 194

§5 Preuve du théorème 1 . . . . . . . . . . . . . . . . . . . . . . . . . . . . . . . . . . . . . . . . . . 195

5.1 L'application du théorème 1 est bien définie . . . . . . . . . . . . . . . . . . . . . . . . 195

5.2 Les cas primitifs classiques . . . . . . . . . . . . . . . . . . . . . . . . . . . . . . . . . . . . . . . . . . . . . 196

5.3 Les cas primitifs non classiques : l'« unicité » . . . . . . . . . . . . . . . . . . . . . . . 197

5.4 Les cas primitifs non classiques : l'« existence » . . . . . . . . . . . . . . . . . . . . . . . . . . 209

5.5 Réduction du cas général au cas primitif . . . . . . . . . . . . . . . . . . . . . . . . . . . . 214

5.6 Résultats complémentaires . . . . . . . . . . . . . . . . . . . . . . . . . . . . . . . 215

$\S 6$ Clôtures sphériques . . . . . . . . . . . . . . . . . . . . . . . . . . . . . . . . . . . . . . 217

6.1 La notion de clôture sphérique . . . . . . . . . . . . . . . . . . . . . . . . . . . . . . . . . . . 217

6.2 Sous-groupes sphériquement clos . . . . . . . . . . . . . . . . . . . . . . . . . . . . 217

6.3 Un résultat intermédiaire . . . . . . . . . . . . . . . . . . . . . . . . . . . . . . . . . . . . . 219

6.4 Caractérisation combinatoire des sous-groupes sphériques ayant une clôture sphérique donnée . . 221

$\S 7$ Preuve du théorème 2 . . . . . . . . . . . . . . . . . . . . . . . . . . . . . . . . . . . . 223

7.1 Clôture sphérique d'un système sphérique . . . . . . . . . . . . . . . . . . . . . . . . 223

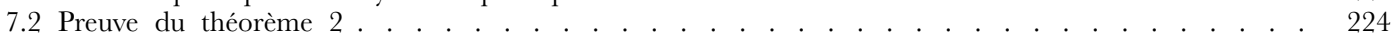

Bibliographie . . . . . . . . . . . . . . . . . . . . . . . . . . . . . . . . . . . . . . . . 225

\section{§1 Rappels sur les variétés sphériques}

Dans toute la suite, $G$ sera un groupe réductif connexe, $B$ un sous-groupe de Borel de $\mathrm{G}$, et $\mathrm{T}$ un tore maximal de $\mathrm{B}$. On identifiera les groupes de caractères $\Xi(\mathbf{B})$ et $\Xi(T)$. On note $R \subset \Xi(B)=\Xi(T)$ le système des racines de $G$, et $S \subset R$ la base de $\mathrm{R}$ associée à $\mathrm{B}$. On désignera par $\mathrm{B}_{-}$le sous-groupe de Borel de $\mathrm{G}$ opposé à $\mathrm{B}$ et contenant $\mathrm{T}$.

Soit $\mathrm{X}$ une $\mathrm{G}$-variété sphérique (c'est-à-dire une G-variété algébrique normale dans laquelle $\mathrm{B}$ a une orbite dense $\mathrm{X}_{\mathrm{B}}^{\circ}$ ). On note $\mathrm{X}_{\mathrm{G}}^{\circ}$ l'orbite ouverte de $\mathrm{G}$ dans $\mathrm{X}$. Le groupe $\mathrm{B}$ (et à plus forte raison aussi le groupe $\mathrm{G}$ ) n'a qu'un nombre fini d'orbites dans $\mathrm{X}$. Toute sous-G-variété irréductible de $\mathrm{X}$ est encore sphérique. On pose $\mathrm{P}_{\mathrm{X}}=\left\{g \in \mathrm{G}, g . \mathrm{X}_{\mathrm{B}}^{\circ}=\mathrm{X}_{\mathrm{B}}^{\circ}\right\}$; c'est un sous-groupe parabolique de $\mathrm{G}$ contenant $\mathrm{B}$. 
Un sous-groupe $H$ de $G$ est dit sphérique si l'espace homogène $G / H$ est (une $\mathrm{G}$-variété) sphérique. Un plongement d'un espace homogène $\mathrm{G} / \mathrm{H}$ est un couple formé d'une $\mathrm{G}$-variété presque homogène $\mathrm{X}$ (d'orbite dense $\mathrm{X}_{\mathrm{G}}^{\circ}$ ) et d'un G-isomorphisme $\mathrm{G} / \mathrm{H} \cong \mathrm{X}_{\mathrm{G}}^{\circ}$.

\section{$\mathbf{1 . 1}$ Espaces vectoriels coloriés ([Kn1], [Br6])}

Soit X une G-variété sphérique.

On désigne par $k(\mathrm{X})$ le corps des fonctions rationnelles de $\mathrm{X}$, et par $\Xi_{\mathrm{X}}$ le sousgroupe de $\Xi(\mathbf{B})$ formé des poids de $\mathrm{B}$ dans $k(\mathrm{X})$. Pour tout $\gamma \in \Xi_{\mathrm{X}}$, choisissons un vecteur propre $f_{\gamma}$ de $\mathbf{B}$ dans $k(\mathbf{X})$ ( $f_{\gamma}$ est unique à scalaire multiplicatif près).

On associe à $\Xi_{X}$ le $\mathbf{Q}$-espace vectoriel $\mathrm{N}_{\mathrm{X}}=\operatorname{Hom}_{\mathbf{Z}}\left(\Xi_{\mathrm{X}}, \mathbf{Q}\right)$.

On appelle rang de $\mathrm{X}$ la dimension de $\mathrm{N}_{\mathrm{X}}$.

On note $\Delta_{\mathrm{X}}$ l'ensemble des diviseurs irréductibles de $\mathrm{X}$ qui sont stables par $\mathrm{B}$, mais non stables par G. Les éléments de $\Delta_{\mathrm{X}}$ sont appelés les couleurs de $\mathrm{X}$ (l'ensemble $\Delta_{\mathrm{X}}$ est en bijection naturelle avec l'ensemble des orbites de codimension 1 de $\mathrm{B}$ dans $\left.\mathrm{X}_{\mathrm{G}}^{\circ}\right)$. On note $\rho_{\mathrm{X}}: \Delta_{\mathrm{X}} \rightarrow \Xi_{\mathrm{X}}^{*}$ l'application définie par :

$$
<\rho_{\mathrm{X}}(\mathrm{D}), \gamma>=v_{\mathrm{D}}\left(f_{\gamma}\right), \gamma \in \Xi_{\mathrm{X}} \text { et } \mathrm{D} \in \Delta_{\mathrm{X}},
$$

où $\Xi_{\mathrm{X}}^{*}$ est le groupe dual de $\Xi_{\mathrm{X}}$ et où $v_{\mathrm{D}}$ désigne la valuation de $k(\mathrm{X})$ associée au diviseur D.

On note $\mathrm{V}_{\mathrm{X}}$ l'ensemble des valuations discrètes $\mathrm{G}$-invariantes (non normalisées et à valeurs dans $\mathbf{Q}$ de $k(\mathrm{X})$ qui s'annulent sur $k^{*}$. L'application qui à $v \in \mathrm{V}_{\mathrm{X}}$ associe l'élément de $\mathrm{N}_{\mathrm{X}}$ défini par $\langle v, \gamma\rangle=v\left(f_{\gamma}\right)\left(\boldsymbol{\gamma} \in \Xi_{\mathrm{X}}\right)$ est injective; on peut donc identifier $\mathrm{V}_{\mathrm{X}}$ à son image dans $\mathrm{N}_{\mathrm{X}}$.

Tous ces invariants $\mathrm{P}_{\mathrm{X}}, \rho_{\mathrm{X}}: \Delta_{\mathrm{X}} \rightarrow \Xi_{\mathrm{X}}^{*} \subset \mathrm{N}_{\mathrm{X}} \supset \mathrm{V}_{\mathrm{X}}$ sont en fait «birationnels» (c'est-à-dire ne dépendent que de $\mathrm{X}_{\mathrm{G}}^{\circ}$ ); autrement dit, si $\mathrm{G} / \mathrm{H} \cong \mathrm{X}_{\mathrm{G}}^{\circ} \subset \mathrm{X}$ est un plongement, alors on a $P_{X}=P_{G / H}, \Xi_{X}=\Xi_{G / H}$ et $\rho_{X}=\rho_{G / H}$ (modulo l'identification naturelle $\Delta_{\mathrm{X}}=\Delta_{\mathrm{G} / \mathrm{H}}$, , etc.

On appelle $\rho_{\mathrm{G} / \mathrm{H}}: \Delta_{\mathrm{G} / \mathrm{H}} \rightarrow \mathrm{N}_{\mathrm{G} / \mathrm{H}} \supset \mathrm{V}_{\mathrm{G} / \mathrm{H}}$ l'espace vectoriel colorié de $\mathrm{G} / \mathrm{H}$; cette notion joue un rôle important dans la théorie des plongements des espaces homogènes sphériques ${ }^{1}{ }^{1}$.

\subsection{Racines sphériques et variétés magnifiques de rang 1 ([Br1], [Wa])}

Soit $\mathrm{X}$ une G-variété sphérique. Un autre invariant (également birationnel), qu'on associe à $\mathrm{X}$, est l'ensemble de ses «racines sphériques». Une première façon de l'introduire est comme suit.

\footnotetext{
$\left.{ }^{1}\right)$ Cette théorie a d'abord été esquissée dans [LV] (voir 8.10, p. 229), puis F. Knop en a donné une version améliorée et complétée dans [Knl]. La meilleure référence est actuellement [Br6].
} 
On montre que le sous-ensemble $\mathrm{V}_{\mathrm{X}}$ de $\mathrm{N}_{\mathrm{X}}$ est un cône convexe simplicial; autrement dit, il existe un sous-ensemble $\Sigma_{\mathrm{X}}$ de $\Xi_{\mathrm{X}}$ vérifiant :

1) $\Sigma_{X}$ est composé d'éléments primitifs de $\Xi_{X}$ linéairement indépendants;

2) $\mathrm{V}_{\mathrm{X}}=\left\{v \in \mathrm{N}_{\mathrm{X}},\langle v, \gamma\rangle \leqslant 0, \gamma \in \Sigma_{\mathrm{X}}\right\}$.

On appelle alors cet ensemble $\Sigma_{\mathrm{X}}$ l'ensemble des racines sphériques de $\mathrm{X}$.

Voici une deuxième manière de procéder.

On commence par définir la notion de variété magnifique de rang 1 : une G-variété algébrique $\mathrm{X}$ est dite magnifique de rang 1 si elle est normale et complète, et si $\mathrm{G}$ a une orbite dense dans $\mathrm{X}$ dont le complémentaire est un diviseur dans lequel $\mathrm{G}$ opère transitivement (autrement dit, $\mathrm{G}$ n'a que deux orbites dans $\mathrm{X}$, une orbite ouverte et dense, et une orbite fermée de codimension 1). On voit facilement que ces variétés sont lisses et sphériques.

Si $\mathrm{X}$ est une $\mathrm{G}$-variété magnifique de rang 1 , notons $z$ l'unique point fixe de $\mathrm{B}_{-}$dans $\mathrm{X}$, et $\mathrm{Z}=\mathrm{G} . z$ l'unique orbite fermée de $\mathrm{G}$ dans $\mathrm{X}$. Alors $\mathrm{T}_{z} \mathrm{X} / \mathrm{T}_{z} \mathrm{Z}$ est un espace vectoriel de dimension 1 dans lequel $\mathrm{T}$ opère. Notons $\gamma_{\mathrm{X}}$ le caractère de $\mathrm{T}$ ainsi obtenu, qu'on appelle la racine sphérique de $\mathrm{X}$. On sait que le couple $\gamma_{\mathrm{X}}, \mathrm{P}_{\mathrm{X}}$ détermine $\mathrm{X}$, à isomorphisme près. Les variétés magnifiques de rang 1 sont toutes connues. En particulier, pour chaque groupe réductif $G$, il n'y a qu'un nombre fini de variétés magnifiques de rang 1, à isomorphisme près. Désignons par $\Sigma(G)$ l'ensemble (fini) des racines sphériques des différentes G-variétés magnifiques de rang 1 . On appelle cet ensemble $\Sigma(G)$ l'ensemble des racines sphériques de $G$.

À titre d'exemple, voici $\Sigma(\mathrm{G})$ pour $\mathrm{G}=\mathrm{PGL}_{n+1}(\mathbf{G})$ (on notera comme d'habitude $\alpha_{1}, \ldots, \alpha_{n}$ les racines simples de $\left.\left.\mathrm{PGL}_{n+1}(\mathbf{G})\right) \quad{ }^{1}\right)$ :

$$
\begin{array}{ll}
\text { type } a_{m} & \gamma=\alpha_{i+1}+\ldots+\alpha_{i+m}(0 \leqslant i \leqslant n-i, 1 \leqslant m \leqslant n-\imath) \\
\text { type } a^{\prime} & \gamma=2 \alpha_{i}(1 \leqslant i \leqslant n) \\
\text { type } d_{3} & \gamma=\alpha_{i}+2 \alpha_{i+1}+\alpha_{i+2}(1 \leqslant i \leqslant n-2) \\
\text { type } a_{1} \times a_{1} & \gamma=\alpha_{i}+\alpha_{j}(1 \leqslant i<j \leqslant n, j-i \geqslant 2) .
\end{array}
$$

Si X est maintenant une $\mathrm{G}$-variété sphérique quelconque, et si $\mathrm{G} / \mathrm{H} \cong \mathrm{X}_{\mathrm{G}}^{\circ}$, on définit l'ensemble $\Sigma_{\mathrm{X}}$ de ses racines sphériques comme l'ensemble des racines sphériques des variétés magnifiques de rang 1 qui se laissent réaliser comme sous-G-variété d'un plongement de $\mathrm{G} / \mathrm{H}$. Il faut vérifier bien sûr que cette deuxième définition coüncide avec la première. L'avantage de cette deuxième définition est qu'elle met en évidence le fait que les racines sphériques de toute $\mathrm{G}$-variété sphérique appartiennent à l'ensemble (fini) $\Sigma(\mathrm{G})$. 
1.3 Variétés magnifiques de rang quelconque ([Lu1])

Une G-variété algébrique $\mathrm{X}$ est appelée magnifique de rang $r$ si elle vérifie les conditions suivantes :

1) $\mathrm{X}$ est lisse et complète;

2) $G$ possède une orbite dense dans $X$ dont le complémentaire est une réunion de diviseurs irréductibles $\mathrm{D}_{i}(i=1, \ldots, r)$, qui sont lisses, à croisements normaux et d'intersection non vide;

3) si $x, x^{\prime} \in \mathrm{X}$ sont tels que $\left\{i, x \in \mathrm{D}_{i}\right\}=\left\{i, x^{\prime} \in \mathrm{D}_{i}\right\}$, alors $\mathrm{G} . x=\mathrm{G} . x^{\prime}$.

Dans une G-variété magnifique (de rang $r$ ), G n'a qu'une seule orbite fermée (de codimension $r$ ). Le radical de $\mathrm{G}$ opère trivialement dans toute $\mathrm{G}$-variété magnifique. Toute sous-G-variété irréductible d'une variété magnifique est encore magnifique.

Toute variété magnifique est sphérique (de rang sphérique égal à son rang comme variété magnifique). Un sous-groupe (sphérique) dont l'espace homogène possède un plongement magnifique sera appelé magnifique. Si $\mathrm{H}$ est un sous-groupe magnifique de $\mathrm{G}$, le groupe $\mathrm{N}_{\mathrm{G}}(\mathrm{H}) / \mathrm{H}$ est fini et le plongement magnifique de $\mathrm{G} / \mathrm{H}$ est unique (à isomorphisme près).

Soit $\mathrm{X}$ une $\mathrm{G}$-variété magnifique. Notons $z$ l'unique point fixe de $\mathrm{B}_{-}$dans $\mathrm{X}$, et $\mathrm{Z}=\mathrm{G} . z$ l'unique orbite fermée de $\mathrm{G}$ dans $\mathrm{X}$. Alors le groupe d'isotropie $\mathrm{G}_{z}$ est aussi le sous-groupe parabolique opposé à $\mathrm{P}_{\mathrm{X}}$ contenant $\mathrm{T}$, et l'ensemble des racines sphériques $\Sigma_{\mathrm{X}}$ est aussi l'ensemble des poids de $\mathrm{T}$ dans $\mathrm{T}_{z} \mathrm{X} / \mathrm{T}_{z} \mathrm{Z}$. De plus, les éléments de $\Sigma_{\mathrm{X}}$ forment une base du groupe abélien $\Xi_{\mathrm{X}}$. Pour tout $\gamma \in \Sigma_{\mathrm{X}}$, notons $\mathrm{D}^{\gamma}$ le diviseur irréductible G-stable (lisse) de $\mathrm{X}$ vérifiant $: \gamma$ est le poids de $\mathrm{T}$ dans $\mathrm{T}_{z} \mathrm{X} / \mathrm{T}_{z} \mathrm{D}^{\gamma}$. Pour tout diviseur $\mathrm{D}$ de $\mathrm{X}$, notons $[\mathrm{D}]$ l'élément de $\mathrm{Pic}(\mathrm{X})$ correspondant. Alors les $[\mathrm{D}]$, $\mathrm{D} \in \Delta_{\mathrm{X}}$ forment une base du $\mathbf{Z}$-module $\operatorname{Pic}(\mathrm{X})$, et

$$
\left[\mathrm{D}^{\gamma}\right]=\sum_{\mathrm{D} \in \Delta_{\mathrm{X}}}<\rho_{\mathrm{X}}(\mathrm{D}), \gamma>[\mathrm{D}] \text {. }
$$

\subsection{Relations entre couleurs et racines simples ([Lu2])}

Pour tout $\mathrm{S}^{\prime} \subset \mathrm{S}$, on désignera par $\mathrm{G}_{\mathrm{S}^{\prime}}\left(\right.$ resp. par $\mathrm{G}_{-\mathrm{S}}$ ) le sous-groupe parabolique associé à $\mathrm{S}^{\prime}$ et contenant $\mathrm{B}$ (resp. $\left.\mathrm{B}_{-}\right)$.

Pour tout $\alpha \in \mathrm{S}$, notons $\alpha^{\mathrm{V}}$ la coracine associée à $\alpha$.

Soit $\mathrm{X}$ une G-variété sphérique. Pour tout $\alpha \in \mathrm{S}$, notons $\Delta_{\mathrm{X}}(\alpha)$ l'ensemble des couleurs de $\mathrm{X}$ qui ne sont pas laissées stables par $\mathrm{G}_{\{\alpha\}}$. Chacun des $\Delta_{\mathrm{X}}(\alpha)$ contient au plus deux éléments. On peut distinguer quatre cas :

1) $\Delta_{\mathrm{X}}(\alpha)=\varnothing$ (cela arrive exactement lorsque $\mathrm{G}_{\{\alpha\}}$ laisse stable $\mathrm{X}_{\mathrm{B}}^{\circ}$ ). On notera $\mathrm{S}_{\mathrm{X}}^{p}$ l'ensemble des $\alpha \in \mathrm{S}$ tels que $\Delta_{\mathrm{X}}(\alpha)=\varnothing$. On a $\mathrm{P}_{\mathrm{X}}=\mathrm{G}_{\mathrm{S}_{\mathrm{X}}^{p}}$. 
2) $\Delta_{\mathrm{X}}(\alpha)$ contient deux éléments (cela arrive exactement lorsque $\alpha \in \Sigma_{\mathrm{X}}$ ). On pose $\mathrm{S}_{\mathrm{X}}^{a}=\mathrm{S} \cap \Sigma_{\mathrm{X}}$. Pour tout $\alpha \in \mathrm{S}_{\mathrm{X}}^{a}$, on note $\mathrm{D}_{\alpha}^{+}$et $\mathrm{D}_{\alpha}^{-}$les deux éléments de $\Delta_{\mathrm{X}}(\alpha)$. On a alors $\left\langle\rho_{\mathrm{X}}\left(\mathrm{D}_{\alpha}^{+}\right), \gamma>+\left\langle\rho_{\mathrm{X}}\left(\mathrm{D}_{\alpha}^{-}\right), \gamma\right\rangle=\left\langle\alpha^{\mathrm{v}}, \gamma>\right.\right.$, quel que soit $\gamma \in \Xi_{\mathrm{X}}$.

3) On a $2 \alpha \in \Sigma_{\mathrm{X}}$ (dans ce cas, $\Delta_{\mathrm{X}}(\alpha)$ contient un seul élément). On pose $\mathrm{S}_{\mathrm{X}}^{a^{\prime}}=\mathrm{S} \cap \frac{1}{2} \Sigma_{\mathrm{X}}$. Pour tout $\alpha \in \mathrm{S}_{\mathrm{X}}^{a^{\prime}}$, on note $\mathrm{D}_{\alpha}^{\prime}$ l'unique élément de $\Delta_{\mathrm{X}}(\alpha)$. On a alors $<\rho_{\mathrm{X}}\left(\mathrm{D}_{\alpha}^{\prime}\right), \gamma>=\frac{1}{2}\left\langle\alpha^{\mathrm{v}}, \gamma>\right.$, quel que soit $\gamma \in \Xi_{\mathrm{X}}$.

4) Le cas qui reste, c'est-à-dire $\Delta_{\mathrm{X}}(\alpha)$ contient un seul élément et $2 \alpha \notin \Sigma_{\mathrm{X}}$. On pose $\mathrm{S}_{\mathrm{X}}^{b}=\mathrm{S} \backslash\left(\mathrm{S}_{\mathrm{X}}^{p} \cup \mathrm{S}_{\mathrm{X}}^{a} \cup \mathrm{S}_{\mathrm{X}}^{a^{\prime}}\right)$. Pour tout $\alpha \in \mathrm{S}_{\mathrm{X}}^{b}$, on note $\mathrm{D}_{\alpha}$ l'unique élément de $\Delta_{\mathrm{X}}(\alpha)$. On a alors $\left\langle\rho_{\mathrm{X}}\left(\mathrm{D}_{\alpha}\right), \gamma\right\rangle=\left\langle\alpha^{\mathrm{v}}, \gamma\right\rangle$, quel que soit $\gamma \in \Xi_{\mathrm{X}}$.

On désignera par $\mathbf{A}_{\mathrm{X}}$ la réunion des $\Delta_{\mathrm{X}}(\alpha), \alpha \in \mathrm{S}_{\mathrm{X}}^{a}=\mathrm{S} \cap \Sigma_{\mathrm{X}}$ (et on notera encore $\rho_{\mathrm{X}}$ la restriction de $\rho_{\mathrm{X}}$ à $\mathbf{A}_{\mathrm{X}}$ ).

\section{\$2 Énoncé des résultats principaux}

On conserve les conventions et notations du $§ 1$. Rappelons que, pour toute racine $\alpha \in \mathrm{R}$, on note $\alpha^{\mathrm{v}}$ la coracine associée.

2.1 Soit $\Sigma$ un sous-ensemble de $\Sigma(\mathrm{G})$ (l'ensemble des racines sphériques de $\mathrm{G}$ ). On notera $\Xi$ le sous-groupe de $\Xi(T)$ engendré par $\Sigma$, et $\Xi^{*}=\operatorname{Hom}_{\mathbf{Z}}(\Xi, \mathbf{Z})$ le groupe dual de $\Xi$.

Soit $\mathbf{A}$ une famille (finie) d'éléments (non nécessairement distincts) de $\Xi^{*}$. Pour tout $\alpha \in \Sigma \cap \mathrm{S}$, on pose $\mathbf{A}(\alpha)=\{\boldsymbol{\delta} \in \mathbf{A}, \delta(\alpha)=1\}$. On dira que la famille $\mathbf{A}$ est adaptée à $\Sigma$, si $\mathbf{A}$ possède les trois propriétés suivantes :

(A1) pour tout $\delta \in \mathbf{A}$ et $\gamma \in \Sigma$, on a $\delta(\gamma) \leqslant 1$, et $\delta(\gamma)=1$ implique $\gamma=\alpha \in \Sigma \cap S$;

(A2) pour tout $\alpha \in \Sigma \cap \mathrm{S}, \mathbf{A}(\alpha)$ contient deux éléments, et si $\mathbf{A}(\alpha)=\left\{\delta_{\alpha}^{+}, \delta_{\alpha}^{-}\right\}$, alors $\delta_{\alpha}^{+}(\gamma)+\delta_{\alpha}^{-}(\gamma)=\left\langle\alpha^{v}, \gamma\right\rangle$, quel que soit $\gamma \in \Sigma$;

(A3) $\mathbf{A}$ est la réunion des $\mathbf{A}(\alpha), \alpha \in \Sigma \cap \mathrm{S}$.

Un triplet $\mathrm{S}^{p}, \Sigma, \mathbf{A}$ formé :

1) d'un sous-ensemble $S^{p}$ de $S$,

2) d'un sous-ensemble $\Sigma$ de $\Sigma(\mathrm{G})$, et

3) d'une famille $\mathbf{A}$ d'éléments de $\Xi^{*}$ adaptée à $\Sigma$,

sera appelé un système sphérique s’il possède les propriétés suivantes.

D'abord deux conditions portant sur $\Sigma$ :

( $\Sigma 1)$ Si $2 \alpha \in \Sigma \cap 2 \mathrm{~S}$, alors $\frac{1}{2}<\alpha^{\mathrm{v}}, \gamma>$ est un entier négatif ou nul, quel que soit $\gamma \in \Sigma \backslash\{2 \alpha\}$.

( $\Sigma 2)$ Si $\alpha+\beta \in \Sigma$ (ou si $\frac{1}{2}(\alpha+\beta) \in \Sigma$ ), où $\alpha, \beta \in \mathrm{S}$ sont orthogonaux, alors $\left.<\alpha^{v}, \gamma\right\rangle=\left\langle\beta^{v}, \gamma>\right.$ quel que soit $\gamma \in \Sigma$. 
Puis une condition portant sur $\Sigma$ et $\mathrm{S}^{p}$ :

(S) Pour tout $\gamma \in \Sigma$, le couple $\gamma, S^{p}$ est celui d'une variété magnifique de rang 1 .

Voici une forme explicite de la condition $(\mathrm{S})$, lorsque le système de racines est de type A :

(S, type A) Pour tout $\gamma \in \Sigma$, on a

$$
\left\{\alpha \in \mathrm{S},<\alpha^{\mathrm{v}}, \gamma>=0\right\} \cap \operatorname{supp}(\gamma) \subset \mathrm{S}^{p} \subset\left\{\alpha \in \mathrm{S},<\alpha^{\mathrm{v}}, \gamma>=0\right\},
$$

où l'on a posé $\operatorname{supp}(\gamma)=\left\{\alpha \in \mathrm{S},<\alpha^{*}, \gamma>\neq 0\right\}$, les $\alpha^{*}(\alpha \in \mathrm{S})$ étant les poids fondamentaux du système des coracines $\mathrm{R}^{\mathrm{v}}$ (rappelons qu'on a $\left\langle\alpha^{*}, \beta>=\delta_{\alpha, \beta}\right.$, quels que soient $\alpha, \beta \in \mathrm{S})$.

\section{Remarques}

1) La notion de système sphérique est purement combinatoire, c'est-à-dire ne dépend que du système des racines de $\mathrm{G}$.

2) Pour tout $G$ fixé, il n'y a qu'un nombre fini de systèmes sphériques. En effet, l'ensemble $\Sigma(\mathrm{G})$ est fini, et des conditions (A1-3) suit que $\operatorname{card}(\mathbf{A}) \leqslant 2 \operatorname{card}(\Sigma \cap \mathrm{S})$, et que $<\alpha^{\mathrm{v}}, \gamma>-1 \leqslant \delta(\gamma) \leqslant 1$, quel que soit $\delta \in \mathbf{A}(\alpha)$ et $\gamma \in \Sigma$.

3) On expliquera plus loin $(\mathrm{au} \S 7)$ que pour toute variété sphérique $\mathrm{X}$, le triplet $\mathrm{S}_{\mathrm{X}}^{p}, \Sigma_{\mathrm{X}}, \mathbf{A}_{\mathrm{X}}$ est un système sphérique.

Voici le résultat principal de ce travail.

Théorème $\mathbf{1}$ - On suppose $G$ semi-simple adjoint de type A. Alors l'application qui, à une $G$-variété magnifique $X$, associe le triplet $\mathrm{S}_{\mathrm{X}}^{p}, \Sigma_{\mathrm{X}}, \mathbf{A}_{\mathrm{X}}$, est une bijection entre l'ensemble (des classes d'isomorphie) des G-variétés magnifiques et l'ensemble des systèmes sphériques de $G$.

Ge théorème sera démontré au §5. Demeure-t-il vrai plus généralement pour tout groupe semi-simple $\mathrm{G}$ ?

2.2 Soit $\mathrm{S}^{p}, \Sigma, \mathbf{A}$ un système sphérique. Il sera souvent commode de considérer $\mathbf{A}$ comme ensemble (abstrait) muni d'une application $\rho: \mathbf{A} \rightarrow \Xi^{*}$.

Un couple $\Xi^{\prime}, \rho^{\prime}$ formé :

1) d'un sous-groupe $\Xi^{\prime}$ de $\Xi(T)$ contenant $\Sigma$ et

2) d'une application $\rho^{\prime}: \mathbf{A} \rightarrow\left(\Xi^{\prime}\right)^{*}$,

sera appelé une augmentation du système sphérique $S^{p}, \Sigma, \mathbf{A}$, s'il vérifie les conditions suivantes :

(a1) $\rho^{\prime}$, suivi de l'application naturelle $\left(\Xi^{\prime}\right)^{*} \rightarrow \Xi^{*}$, est égal à $\rho$;

(a2) pour tout $\alpha \in \Sigma \cap S$, si $\mathbf{A}(\alpha)=\left\{\delta_{\alpha}^{+}, \delta_{\alpha}^{-}\right\}$, alors $\left\langle\rho^{\prime}\left(\delta_{\alpha}^{+}\right), \gamma\right\rangle+\left\langle\rho^{\prime}\left(\boldsymbol{\delta}_{\alpha}^{-}\right), \gamma\right\rangle$ $=\left\langle\alpha^{\mathrm{v}}, \gamma\right\rangle$ quel que soit $\gamma \in \Xi^{\prime}$ 
$(\sigma 1)$ si $2 \alpha \in \Sigma \cap 2 S$, alors $\alpha \notin \Xi^{\prime}$ et $\left\langle\alpha^{v}, \gamma\right\rangle$ est un entier pair, quel que soit $\gamma \in \Xi^{\prime}$

( $\sigma 2)$ si $\alpha+\beta \in \Sigma$ (ou si $\frac{1}{2}(\alpha+\beta) \in \Sigma$ ), où $\alpha, \beta \in$ S sont orthogonaux, alors $\left.<\alpha^{\mathrm{v}}, \gamma\right\rangle=\left\langle\beta^{\mathrm{v}}, \gamma>\right.$ quel que soit $\gamma \in \Xi^{\prime}$

(s) pour tout $\alpha \in \mathrm{S}^{p}, \alpha^{v}$ s'annule sur $\Xi^{\prime}$.

On dira aussi que le quintuplet $\mathrm{S}^{p}, \Sigma, \mathbf{A}, \Xi^{\prime}, \rho^{\prime}$ est un système sphérique augmenté. Un système sphérique augmenté tel que tout élément de $\Sigma$ est primitif dans $\Xi^{\prime}$ sera appelé une donnée sphérique homogène.

Théorème 2 - On suppose que le théorème 1 est vrai pour le groupe adjoint de G. Alors l'application qui, à une $G$-variété homogène sphérique $X$, associe le quintuplet $\mathrm{S}_{\mathrm{X}}^{p}, \Sigma_{\mathrm{X}}, \mathbf{A}_{\mathrm{X}}, \Xi_{\mathrm{X}}$, $\rho_{\mathrm{X}}$ est une bijection entre l'ensemble (des classes d'isomorphie) des $G$-variétés homogènes sphériques et l'ensemble des données sphériques homogènes de $G$.

Ce théorème sera démontré au $§ 7$.

2.3 Soit $\mathrm{S}^{p}, \Sigma, \mathbf{A}, \Xi^{\prime}, \rho^{\prime}$ un système sphérique augmenté. Nous allons lui associer, de façon naturelle, un ensemble de «couleurs» et un «espace vectoriel colorié».

$$
\begin{aligned}
& \text { Posons } \\
& \mathrm{S}^{a}=\mathrm{S} \cap \Sigma, \\
& \mathrm{S}^{a^{\prime}}=\{\alpha \in \mathrm{S}, 2 \alpha \in \Sigma\}, \text { et } \\
& \mathrm{S}^{b}=\mathrm{S} \backslash\left(\mathrm{S}^{a} \cup \mathrm{S}^{a^{\prime}} \cup \mathrm{S}^{p}\right) ;
\end{aligned}
$$

puis posons

$\Delta^{a}=\mathbf{A}$

$\Delta^{a^{\prime}}=\mathrm{S}^{a^{\prime}}$, et

$\Delta^{b}=\mathrm{S}^{b} / \sim\left(\sim\right.$ signifiant qu'on identifie $\alpha, \beta \in \mathrm{S}^{b}$, s'ils sont orthogonaux et si $\alpha+\beta \in \Sigma\left(\right.$ ou si $\left.\frac{1}{2}(\alpha+\beta) \in \Sigma\right)$ ); enfin posons

$$
\Delta=\Delta^{a} \cup \Delta^{a^{\prime}} \cup \Delta^{b} .
$$

Si $\alpha \in \mathrm{S}^{a^{\prime}}$, notons $\delta_{\alpha}^{\prime}$ l'élément de $\Delta^{a^{\prime}}$ qui lui correspond; si $\alpha \in \mathrm{S}^{b}$, notons $\delta_{\alpha}$ l'image de $\alpha$ dans $\Delta^{b}$. Définissons une application $\Delta \rightarrow\left(\Xi^{\prime}\right)^{*}$, encore notée $\rho^{\prime}$, de la façon suivante :

sur $\Delta^{a}$ par le $\rho^{\prime}$ déjà existant sur $\Delta^{a}=\mathbf{A}$;

$\operatorname{sur} \Delta^{a^{\prime}} \operatorname{par}\left\langle\rho^{\prime}\left(\delta_{\alpha}^{\prime}\right), \gamma>=\frac{1}{2}<\alpha^{\mathrm{v}}, \gamma>\left(\alpha \in \mathrm{S}^{a^{\prime}}, \gamma \in \Xi^{\prime}\right)\right.$;

sur $\Delta^{b}$ par $\left\langle\rho^{\prime}\left(\delta_{\alpha}\right), \gamma\right\rangle=\left\langle\alpha^{\mathrm{v}}, \gamma\right\rangle\left(\alpha \in \mathrm{S}^{b}, \gamma \in \Xi^{\prime}\right)$.

On appellera le couple $\Delta, \rho^{\prime}$ l'ensemble des couleurs du système sphérique augmenté $\mathrm{S}^{p}$, $\Sigma, \mathbf{A}, \Xi^{\prime}, \rho^{\prime}$. 
Posons $\mathrm{N}^{\prime}=\operatorname{Hom}_{\mathbf{Z}}\left(\Xi^{\prime}, \mathbf{Q}\right)$ (espace vectoriel qui contient $\left.\left(\Xi^{\prime}\right)^{*}\right)$, et désignons par $\mathrm{V}^{\prime}$ l'ensemble (le cône convexe) des éléments de $\mathrm{N}^{\prime}$ qui sont $\leqslant 0$ sur $\Sigma$. On appellera $\rho^{\prime}: \Delta \rightarrow \mathrm{N}^{\prime} \supset \mathrm{V}^{\prime}$ l'espace vectoriel colorié du système sphérique augmenté $\mathrm{S}^{p}, \Sigma, \mathbf{A}, \Xi^{\prime}, \rho^{\prime}$.

Nous ne rappellerons pas ici la définition des éventails coloriés (pour cela, ainsi que pour tout ce qui concerne la théorie des plongements des espaces homogènes sphériques, voir [Knl] ou [Br6]). Par éventail colorié d'un système sphérique augmenté, on entend bien sûr un éventail colorié de son espace vectoriel colorié associé.

Théorème 3 - On suppose que le théorème 1 est vrai pour le groupe adjoint de G. Alors l'application qui, à une G-variété sphérique, associe sa donnée sphérique et son éventail colorié est une bijection entre l'ensemble (des classes d'isomorphie) des G-variétés sphériques et l'ensemble des couples formés

1) d'une donnée sphérique homogène de $G$ et

2) d'un éventail colorié de cette donnée sphérique.

Preuve - Pour tout espace homogène sphérique $\mathrm{G} / \mathrm{H}$, son espace vectoriel colorié s'identifie à l'espace vectoriel colorié de la donnée sphérique homogène associée à $\mathrm{G} / \mathrm{H}$ (cela résulte de 1.4 et de la proposition 3.2). Le théorème 3 est alors une conséquence immédiate du théorème 2 et de la théorie des plongements des espaces homogènes sphériques.

\section{§3 Variétés magnifiques et systèmes sphériques}

Dans ce paragraphe, on étudiera les relations entre la géométrie des variétés magnifiques et la combinatoire de leurs systèmes sphériques. Sauf mention expresse du contraire, les données (systèmes sphériques et groupes) seront supposées adjointes (c'està-dire, en ce qui concerne les systèmes sphériques, composées de racines sphériques appartenant au réseau radiciel).

\subsection{Sous-systèmes}

Commençons par introduire un peu de terminologie concernant les systèmes sphériques.

Soit $\mathrm{S}$ une base d'un système de racines, et soit $\mathrm{S}^{p}, \Sigma, \mathbf{A}$ un système sphérique (de S). Rappelons qu'on note $\Xi$ le sous-groupe (du réseau radiciel) engendré par $\Sigma$.

Si $\Sigma^{\prime} \subset \Sigma$, on notera supp $\Sigma^{\prime}$ la réunion des supp $\gamma, \gamma \in \Sigma^{\prime}$ (pour la définition de supp $\gamma$, voir 2.1). Si $\mathrm{S}^{\prime} \subset \mathrm{S}$, on notera $\mathbf{A}\left(\mathrm{S}^{\prime}\right)$ la réunion $\operatorname{des} \mathbf{A}(\alpha), \alpha \in \mathrm{S}^{\prime}$.

Soit $\left(\mathrm{S}^{\prime}, \Sigma^{\prime}\right)$ un couple (avec $\mathrm{S}^{\prime} \subset \mathrm{S}$ et $\left.\Sigma^{\prime} \subset \Sigma\right)$ vérifiant supp $\Sigma^{\prime} \subset \mathrm{S}^{\prime}$. Le soussystème de $S^{p}, \Sigma, \mathbf{A}$ associé au couple $\left(\mathrm{S}^{\prime}, \Sigma^{\prime}\right)$ est par définition le système sphérique (de $\left.\mathrm{S}^{\prime}\right)$ donné par $\mathrm{S}^{\prime p}=\mathrm{S}^{\prime} \cap \mathrm{S}^{p}$, $\Sigma^{\prime}$, et $\mathbf{A}^{\prime}=$ la «restriction» de $\mathbf{A}\left(\mathrm{S} \cap \Sigma^{\prime}\right)$ à $\Xi^{\prime}$ (le sous-groupe de $\Xi$ engendré par $\left.\Sigma^{\prime}\right)$. Soulignons qu'il y a perte d'information dans le passage de $\mathbf{A}\left(\mathrm{S} \cap \Sigma^{\prime}\right)$ à $\mathbf{A}^{\prime}$. 
Nous rencontrerons deux cas particuliers :

Si $\mathrm{S}^{\prime} \subset \mathrm{S}$, on dira que le sous-système associé au couple $\left(\mathrm{S}^{\prime}, \Sigma^{\prime}=\{\gamma \in \Sigma\right.$, $\left.\operatorname{supp} \gamma \subset \mathrm{S}^{\prime}\right\}$ ) est obtenu par localisation en $\mathrm{S}^{\prime}$.

Si $\Sigma^{\prime} \subset \Sigma$, on dira que le sous-système associé au couple $\left(\mathrm{S}, \Sigma^{\prime}\right)$ est obtenu par localisation en $\Sigma^{\prime}$.

Un système sphérique $\mathrm{S}^{p}, \Sigma, \mathbf{A}$ sera appelé cuspidal si $\operatorname{supp} \Sigma=\mathrm{S}$.

Une factorisation d'un système sphérique $\mathrm{S}^{p}, \Sigma, \mathbf{A}$ est une partition de $\mathrm{S}$ en deux sous-ensembles (non vides) $S_{1}$ et $S_{2}$ vérifiant :

a) (tout élément de) $\mathrm{S}_{1}$ est orthogonal à (tout élément de) $\mathrm{S}_{2}$;

b) si $\Sigma_{i}=\left\{\gamma \in \Sigma, \operatorname{supp}(\gamma) \subset \mathrm{S}_{i}\right\}(i=1,2)$, alors $\Sigma=\Sigma_{1} \cup \Sigma_{2}$;

c) pour tout $\delta \in \mathbf{A}\left(\Sigma \cap \mathrm{S}_{1}\right)$, $\delta$ est nul sur $\Sigma_{2}$, et pour tout $\delta \in \mathbf{A}\left(\Sigma \cap \mathrm{S}_{2}\right)$, $\delta$ est nul $\operatorname{sur} \Sigma_{1}$.

Toute décomposition d'une $G$-variété magnifique $X$ «en produit» $G=G_{1} \times G_{2}$ et $\mathrm{X}=\mathrm{X}_{1} \times \mathrm{X}_{2}$ (où les $\mathrm{X}_{i}$ sont des $\mathrm{G}_{i}$-variétés magnifiques, $i=1,2$ ) factorise son système sphérique, et l'inverse est également vrai (voir 3.5).

Un système sphérique sera dit irréductible s'il ne possède pas de factorisation. Il est clair que tout système sphérique se laisse factoriser, de façon unique, en facteurs irréductibles.

\subsection{Localisation ([Lu2])}

Soit $\mathrm{X}$ une G-variété magnifique, et soit $\mathrm{S}^{p}, \Sigma, \mathbf{A}$ le système sphérique de $\mathrm{X}$. Soit $\mathrm{S}^{\prime} \subset \mathrm{S}$. Désignons par $\mathrm{G}_{\mathrm{S}^{\prime}}\left(\right.$ resp. $\mathrm{G}_{-\mathrm{S}^{\prime}}$ ) le sous-groupe parabolique de $\mathrm{G}$ contenant $B$ (resp. $B_{-}$) et associé à $S^{\prime}$. Posons $G^{\prime}=G_{S^{\prime}} \cap G_{-S^{\prime}}\left(=G^{S^{\prime}}\right)$; c'est un sous-groupe de Levi de $\mathrm{G}_{\mathrm{S}^{\prime}}$ et de $\mathrm{G}_{-\mathrm{S}^{\prime}}$. Notons $\mathrm{C}^{\prime}\left(=\mathrm{C}^{\mathrm{S}^{\prime}}\right.$ ) le centre connexe (le radical) de $\mathrm{G}^{\prime}$. Enfin, désignons par $\mathrm{X}^{\prime}\left(=\mathrm{X}^{\mathrm{S}^{\prime}}\right)$ la composante connexe de $\mathrm{X}^{\mathrm{C}^{\prime}}$ contenant $z$ (l'unique point fixe de $\mathrm{B}_{-}$dans $\left.\mathrm{X}\right)$. Alors la $\mathrm{G}^{\prime}$-variété $\mathrm{X}^{\prime}$ est magnifique.

Posons $\mathrm{X}_{\mathrm{S}^{\prime}}=\left\{x \in \mathrm{X}, \overline{\mathrm{G}_{\mathrm{S}^{\prime}} \cdot x} \ni z\right\}$; c'est un sous-ensemble ouvert de $\mathrm{X}$, stable par $\mathrm{G}_{\mathrm{S}^{\prime}}$. On a $\mathrm{X}^{\prime} \subset \mathrm{X}_{\mathrm{S}^{\prime}}$, et l'application $\phi: \mathrm{X}_{\mathrm{S}^{\prime}} \rightarrow \mathrm{X}^{\prime}$ définie par $\phi(x)=$ l'unique point de $\overline{\mathrm{C}^{\prime} . x} \cap \mathrm{X}^{\prime}$ est un morphisme lisse (une rétraction). Posons $\Delta_{\mathrm{X}}\left(\mathrm{S}^{\prime}\right)=$ la réunion des $\Delta_{\mathrm{X}}(\alpha), \alpha \in \mathrm{S}^{\prime}$. Alors l'application $\mathrm{D}^{\prime} \in \Delta_{\mathrm{X}^{\prime}} \rightarrow \overline{\phi^{-1}\left(\mathrm{D}^{\prime}\right)}$ est un bijection de $\Delta_{\mathrm{X}^{\prime}}$ sur $\Delta_{\mathrm{X}}\left(\mathrm{S}^{\prime}\right)$ (l'application inverse est $\left.: \mathrm{D} \in \Delta_{\mathrm{X}}\left(\mathrm{S}^{\prime}\right) \rightarrow \mathrm{D} \cap \mathrm{X}^{\prime}\right)$.

On peut considérer $\mathrm{S}^{\prime}$ comme base du système de racines de $\mathrm{G}^{\prime}$ (associée au sousgroupe de Borel $\mathrm{B} \cap \mathrm{G}^{\prime}$ de $\mathrm{G}^{\prime}$ ), et identifier $\Sigma\left(\mathrm{G}^{\prime}\right)$ (l'ensemble des racines sphériques de $\left.\mathrm{G}^{\prime}\right)$ à $\left\{\gamma \in \Sigma(\mathrm{G}), \operatorname{supp}(\gamma) \subset \mathrm{S}^{\prime}\right\}$. Modulo cette identification, le système sphérique de la $\mathrm{G}^{\prime}$-variété magnifique $\mathrm{X}^{\prime}$ s'obtient, partant du système sphérique de $\mathrm{X}$, par localisation en $\mathrm{S}^{\prime}$ (qu'on vient de définir dans la section précédente).

On dira aussi que $\mathrm{X}^{\prime}$ est obtenu, partant de $\mathrm{X}$, par localisation en $\mathrm{S}^{\prime}$. La localisation est une méthode efficace pour obtenir des résultats sur les invariants des variétés magnifiques. À titre d'exemple, montrons la propriété suivante. 
Proposition 3.2 - Soit $G$ un groupe réductif connexe, et soit $X$ une $G$-variété sphérique. Si $\alpha, \beta \in \mathrm{S}$, alors $\Delta_{\mathrm{X}}(\alpha) \cap \Delta_{\mathrm{X}}(\boldsymbol{\beta}) \neq \varnothing$ ne se produit que dans deux cas :

a) si $\alpha$ et $\beta \in \mathrm{S} \cap \Sigma_{\mathrm{X}}$, alors il peut arriver que card $\Delta_{\mathrm{X}}(\alpha) \cup \Delta_{\mathrm{X}}(\beta)=3$;

b) si $\alpha$ est orthogonal à $\beta$ et si $\alpha+\beta$ (ou sa moitié) appartient à $\Sigma_{\mathrm{X}}$, alors $\mathrm{D}_{\alpha}=\mathrm{D}_{\beta}$.

Preuve - Il suffit de considérer le cas où $\mathrm{X}$ est un espace homogène G/H. $\mathrm{Si} \overline{\mathrm{H}}$ désigne la clôture sphérique de $\mathrm{H}$ (voir §6), à cause des bijections naturelles $\Delta_{\mathrm{G} / \mathrm{H}}(\alpha) \rightarrow \Delta_{\mathrm{G} / \overline{\mathrm{H}}}(\alpha)(\alpha \in \mathrm{S})$, on est ramené au cas $\mathrm{G} / \overline{\mathrm{H}}$. Mais cet espace homogène possède un plongement magnifique ([Kn2]), donc on est ramené au cas $\mathrm{G}$ adjoint et $\mathrm{X}$ magnifique.

Il suffit alors de vérifier la proposition après localisation en $S^{\prime}=\{\alpha, \beta\}$, c'està-dire lorsque le groupe est semi-simple de rang 2, auquel cas on connaît toutes les variétés magnifiques et la vérification se fait facilement, cas par cas (voir par exemple les tables de [Wa]).

Soit $\mathrm{X}$ une variété magnifique. Pour tout $\Sigma^{\prime} \subset \Sigma_{\mathrm{X}}$, désignons par $\mathrm{X}\left(\Sigma^{\prime}\right)$ l'intersection des $\mathrm{D}^{\gamma}, \gamma \in \Sigma \backslash \Sigma^{\prime}$ (pour la définition de $\mathrm{D}^{\gamma}$, voir 1.3); c'est une sous$\mathrm{G}$-variété magnifique de $\mathrm{X}$, et toutes s'obtiennent ainsi. On vérifie que le système sphérique de $\mathrm{X}\left(\Sigma^{\prime}\right)$ s'obtient à partir du système sphérique de $\mathrm{X}$, par localisation en $\Sigma^{\prime}$ (pour relier $\mathbf{A}_{\mathbf{X}\left(\Sigma^{\prime}\right)}$ à $\mathbf{A}$, on peut utiliser par exemple [Lu2], 3.5).

\subsection{Sous-ensembles distingués de couleurs et quotients} colorié.

Soit $\mathrm{S}^{p}, \Sigma, \mathbf{A}$ un système sphérique et soit $\rho: \Delta \rightarrow \mathrm{N} \supset \mathrm{V}$ son espace vectoriel

Un sous-ensemble $\Delta^{\prime} \subset \Delta$ sera dit distingué si $\mathrm{C}\left(\rho\left(\Delta^{\prime}\right)\right)^{\circ}$ (l'intérieur relatif du cône convexe engendré par $\rho\left(\Delta^{\prime}\right)$ ) rencontre le cône (convexe) $-\mathrm{V}$.

Lemme 3.3.1 - Pour qu'un sous-ensemble $\Delta^{\prime} \subset \Delta$ soit distingué, il faut et il suffit qu'il existe un sous-espace vectoriel $\mathrm{N}^{\prime}=\mathrm{N}\left(\Delta^{\prime}\right)$ (unique) de $\mathrm{N}$ vérifiant :

1) le couple $\mathrm{N}^{\prime}, \Delta^{\prime}$ est un sous-espace vectoriel colorié (c'est-à-dire $\rho\left(\Delta^{\prime}\right)$ et $\mathrm{N}^{\prime} \cap \mathrm{V}$ engendrent $\mathrm{N}^{\prime}$ comme cône convexe);

2) Lintersection $\mathrm{N}^{\prime} \cap \mathrm{V}$ est une facette du cône $\mathrm{V}$.

Preuve - Si $\Delta^{\prime} \subset \Delta$ est distingué, soit $\mathrm{F}^{\prime}$ la plus petite facette de $\mathrm{V}$ vérifiant $\mathrm{C}\left(\rho\left(\Delta^{\prime}\right)\right)^{\circ} \cap\left(-\mathrm{F}^{\prime}\right) \neq \varnothing$. Alors le cône convexe $\mathrm{N}^{\prime}$ engendré par $\rho\left(\Delta^{\prime}\right)$ et $\mathrm{F}^{\prime}$ est un sousespace vectoriel qui vérifie les conditions (1) et (2).

Inversement, soit $\mathrm{N}^{\prime}$ un sous-espace vectoriel de $\mathrm{N}$ qui vérifie les conditions (1) et (2), et posons $\mathrm{F}^{\prime}=\mathrm{N}^{\prime} \cap \mathrm{V}$. D'après (2), on obtient ainsi une facette de $\mathrm{V}$, et (1) implique que $\mathrm{C}\left(\rho\left(\Delta^{\prime}\right)\right)^{\circ} \cap(-\mathrm{V}) \neq \varnothing$. L'unicité de $\mathrm{N}^{\prime}$ vient du fait qu'il est visiblement le sous-espace vectoriel engendré par la plus petite facette $\mathrm{F}^{\prime}$ qui vérifie $\mathrm{C}\left(\rho\left(\Delta^{\prime}\right)\right)^{\circ} \cap\left(-\mathrm{F}^{\prime}\right) \neq \varnothing$. 
Soit $\mathrm{S}^{p}, \Sigma, \mathbf{A}$ un système sphérique et soit $\Delta^{\prime}$ un sous-ensemble distingué de $\Delta$. Notons $\Xi / \Delta^{\prime}$ le sous-groupe des éléments de $\Xi$ annulés par tout élément de $\mathrm{N}\left(\Delta^{\prime}\right)$. Dans ce qui suit on va définir un triplet quotient $\left(\mathrm{S}^{p}, \Sigma, \mathbf{A}\right) / \Delta^{\prime}$ :

a) Posons $\mathrm{S}^{p} / \Delta^{\prime}=\left\{\alpha \in \mathrm{S}, \Delta(\alpha) \subset \Delta^{\prime}\right\}$.

b) Les combinaisons linéaires $\sum_{\gamma \in \Sigma} n(\gamma) \gamma$ (où les $n(\gamma)(\gamma \in \Sigma)$ sont des entiers $\geqslant 0$ tels que $\sum_{\gamma \in \Sigma} n(\gamma) \gamma$ est dans $\left.\Xi / \Delta^{\prime}\right)$ forment un semi-groupe additif; notons $\Sigma / \Delta^{\prime}$ l'ensemble des éléments indécomposables de ce semi-groupe.

c) Désignons par $\mathbf{A} / \Delta^{\prime}$ la réunion des $\mathbf{A}(\alpha), \alpha \in \mathrm{S} \cap \Sigma$ tels que $\mathbf{A}(\alpha) \cap \Delta^{\prime}=\varnothing$, et notons $\rho / \Delta^{\prime}: \mathbf{A} / \Delta^{\prime} \rightarrow\left(\Xi / \Delta^{\prime}\right)^{*}$ la restriction de $\rho$ à $\mathbf{A} / \Delta^{\prime}$ suivi de l'application naturelle $\Xi^{*} \rightarrow\left(\Xi / \Delta^{\prime}\right)^{*}$.

On dira que $\Delta^{\prime}$ possède la propriété $\left(^{*}\right)$ si les éléments de $\Sigma / \Delta^{\prime}$ forment une base du Z-module $\Xi / \Delta^{\prime}$.

Remarque - Lorsque $\mathrm{S}$ est de type A, on verra au $\S 5, \mathrm{n}^{\circ} 6$, que :

1) tous les sous-ensembles distingués possèdent la propriété $\left(^{*}\right)$;

2) leur triplet quotient est encore un système sphérique.

Pour les sous-ensembles distingués qu'on rencontrera par la suite, (1) et (2) se vérifient facilement dans chaque cas.

Soit $\mathrm{X}$ une G-variété magnifique, et soit $\mathrm{S}^{p}, \Sigma, \mathbf{A}$ le système sphérique de $\mathrm{X}$. Soit $\mathrm{X}^{\prime}$ une deuxième G-variété magnifique, et soit $\phi: \mathrm{X} \rightarrow \mathrm{X}^{\prime}$ un G-morphisme dominant. Posons $\Delta(\phi)=\left\{\mathrm{D} \in \Delta_{\mathrm{X}}, \phi(\mathrm{D})=\mathrm{X}^{\prime}\right\}$.

\section{Proposition 3.3.2}

1) L'application qui à $\phi$ associe $\Delta(\phi)$, est une bijection entre l'ensemble des $G$-morphismes dominants à fibres connexes de $X$ dans une autre G-variété magnifique, et l'ensemble des sous-ensembles distingués de $\Delta$ vérifiant la propriété (*).

2) Le système sphérique de $\mathrm{X}^{\prime}$ est $\left(\mathrm{S}^{p}, \Sigma, \mathbf{A}\right) / \Delta(\phi)$.

Preuve - Posons $\mathrm{G} / \mathrm{H}=\mathrm{X}_{\mathrm{G}}^{\circ}$. D'après la théorie des plongements des espaces homogènes sphériques, on sait qu'il y a une bijection entre les G-morphismes à fibres connexes $\mathrm{G} / \mathrm{H} \rightarrow \mathrm{G} / \mathrm{H}^{\prime}$, et les sous-espaces vectoriels coloriés $\mathrm{N}^{\prime}, \Delta^{\prime}$ de $\rho: \Delta \rightarrow \mathrm{N} \supset \mathrm{V}$ (voir $[\mathrm{Knl}]$ ou [Br6]). Pour que $\mathrm{G} / \mathrm{H}^{\prime}$ possède un plongement magnifique, il faut et il suffit que $\mathrm{N}^{\prime} \cap \mathrm{V}$ soit une facette du cône $\mathrm{V}$ (donc que $\Delta^{\prime}$ soit distingué et que $\mathrm{N}^{\prime}=\mathrm{N}\left(\Delta^{\prime}\right)$ ), et que $\Delta^{\prime}$ vérifie la condition $\left(^{*}\right)$. Le morphisme $\mathrm{G} / \mathrm{H} \rightarrow \mathrm{G} / \mathrm{H}^{\prime}$ se prolonge alors (par continuité) en un G-morphisme $\phi: \mathrm{X} \rightarrow \mathrm{X}^{\prime}$ (vérifiant $\Delta^{\prime}=\Delta(\phi)$ ), d'où 1 ). De plus, l'assertion 2) est alors vraie par construction (voir loc. cit.). 
On dira d'un sous-ensemble distingué $\Delta^{\prime} \subset \Delta$ :

qu'il est lisse, si la dimension de $\mathrm{N}\left(\Delta^{\prime}\right)$ est égale à la dimension de la facette $\mathrm{N}\left(\Delta^{\prime}\right) \cap \mathrm{V}$; qu'il est parabolique, si $\mathrm{N}\left(\Delta^{\prime}\right)=\mathrm{N}$.

\section{Proposition 3.3.3}

1) Soient $\mathrm{X}$ et $\mathrm{X}^{\prime}$ deux G-variétés magnifiques, et soit $\phi: \mathrm{X} \rightarrow \mathrm{X}^{\prime}$ un G-morphisme dominant à fibres connexes. Alors $\phi$ est lisse si et seulement si $\Delta(\phi)$ l'est.

2) Soit $\mathrm{X}$ une $G$-variété magnifique et soit $\mathrm{S}^{\prime} \subset \mathrm{S}$. L'application qui à $\phi$ associe $\Delta(\phi)$, est une bijection entre l'ensemble des $\mathrm{G}$-morphismes $\phi: \mathrm{X} \rightarrow \mathrm{G} / \mathrm{G}_{-\mathrm{S}^{\prime}}$, et l'ensemble des sous-ensembles distingués paraboliques $\Delta^{\prime} \subset \Delta$ tels que $\mathrm{S}^{\prime}=\mathrm{S} \backslash\left(\mathrm{S}^{p} / \Delta^{\prime}\right)$.

Preuve - 1) Notons $z$ et $z^{\prime}$ les points fixes de $\mathrm{B}_{-}$dans $\mathrm{X}$ et $\mathrm{X}^{\prime}$, et $\mathrm{Z}=\mathrm{G} . z$ et $\mathrm{Z}^{\prime}=\mathrm{G} . z^{\prime}$ les orbites fermées de $\mathrm{G}$ dans $\mathrm{X}$ et $\mathrm{X}^{\prime}$. Le morphisme $\phi$ est lisse si et seulement si l'application naturelle

(*) $\mathrm{T}_{z} \mathrm{X} / \mathrm{T}_{z} \mathrm{Z} \rightarrow \mathrm{T}_{z^{\prime}} \mathrm{X}^{\prime} / \mathrm{T}_{z^{\prime}} \mathrm{Z}^{\prime}$ est surjective.

Par ailleurs, $\Delta(\phi)$ est lisse si et seulement si

(**) $\Sigma / \Delta(\phi)$ est un sous-ensemble de $\Sigma$.

Puisque $\Sigma$ (resp. $\Sigma / \Delta(\phi))$ est l'ensemble des poids de $\mathrm{T}$ dans $\mathrm{T}_{z} \mathrm{X} / \mathrm{T}_{z} \mathrm{Z}$ (resp. dans $\left.\mathrm{T}_{z^{\prime}} \mathrm{X}^{\prime} / \mathrm{T}_{z^{\prime}} \mathrm{Z}^{\prime}\right),\left({ }^{*}\right)$ implique (**). On sait que les fonctions rationnelles $f_{-\gamma}, \gamma \in \Sigma$ (resp. les $\left.f_{-\gamma}, \gamma \in \Sigma / \Delta(\phi)\right)$ forment un système de paramètres de $\mathrm{Z}$ dans $\mathrm{X}$ au point $z$ (resp. de $\mathrm{Z}^{\prime}$ dans $\mathrm{X}^{\prime}$ au point $z^{\prime}$ ), voir par exemple [Lu2] 2.6; par suite $\left(^{* *}\right)$ entraine aussi $\left(^{*}\right)$.

2) Un sous-ensemble distingué $\Delta^{\prime} \subset \Delta$ est parabolique si et seulement si $\Sigma / \Delta^{\prime}=\varnothing$. Gela et 3.3.2 impliquent aussitôt 2).

\subsection{Induction parabolique}

Soit $\mathrm{Q}$ un sous-groupe parabolique de $\mathrm{G}$ contenant $\mathrm{B}_{-}$(il existe donc un $\mathrm{S}^{\prime} \subset \mathrm{S}$ unique tel que $Q=\mathrm{G}_{-\mathrm{S}^{\prime}}$; rappelons qu'on a posé $\left.\mathrm{G}^{\prime}=\mathrm{G}_{\mathrm{S}^{\prime}} \cap \mathrm{G}_{-\mathrm{S}^{\prime}}\right)$.

Soit X une G-variété magnifique. On dira qu'un G-morphisme $\phi: X \rightarrow G / Q$ provient d'une induction parabolique si $\mathrm{Q}^{r}$ (le radical de $\mathrm{Q}$ ) opère trivialement dans $\mathrm{X}^{\prime}=\phi^{-1}(\mathrm{Q} / \mathrm{Q})$. Alors $\mathrm{X}^{\prime}$ est une $\mathrm{G}^{\prime}$-variété magnifique, et $\mathrm{X}$ est canoniquement isomorphe à $\mathrm{G} *_{\mathrm{Q}} \mathrm{X}^{\prime}$ (on note ainsi la $\mathrm{G}$-variété algébrique obtenue comme quotient de $\mathrm{G} \times \mathrm{X}^{\prime}$ sous l'opération de $\mathrm{Q}$ donnée par

$$
\left.q \cdot(g, x)=\left(g q^{-1}, q \cdot x\right), q \in \mathrm{Q}, g \in \mathrm{G}, x \in \mathrm{X}^{\prime}\right) .
$$

Inversement, si $\mathrm{X}^{\prime}$ est une $\mathrm{G}^{\prime}$-variété magnifique (qu'on peut considérer comme une Q-variété, en faisant opérer $\mathrm{Q}^{r}$ trivialement, étant donné que $\left.\mathrm{Q}=\mathrm{Q}^{r} \mathrm{G}^{\prime}\right)$, alors $\mathrm{G} *_{\mathrm{Q}} \mathrm{X}^{\prime}$ est une G-variété magnifique, et le morphisme naturel $\mathrm{G} *_{\mathrm{Q}} \mathrm{X}^{\prime} \rightarrow \mathrm{G} / \mathrm{Q}$ provient 
d'une induction parabolique. On dira alors aussi que $\mathrm{X}=\mathrm{G} *_{\mathrm{Q}} \mathrm{X}^{\prime}$ est obtenu par induction parabolique (à travers $\mathrm{Q}$ au moyen de $\mathrm{X}^{\prime}$ ). On vérifie sans peine que, modulo les inclusions $\mathrm{S}^{\prime} \subset \mathrm{S}$ et $\Sigma\left(\mathrm{G}^{\prime}\right) \subset \Sigma(\mathrm{G})$, $\mathrm{X}$ et $\mathrm{X}^{\prime}$ ont le «même» système sphérique (l'un étant un système dans $\mathrm{S}$ et l'autre dans $\left.\mathrm{S}^{\prime}\right)$.

Soit $\mathrm{X}$ une $\mathrm{G}$-variété magnifique, et soit $\mathrm{S}^{\prime} \subset \mathrm{S}$. Notons $\mathrm{S}^{p}, \Sigma$, A le système sphérique de $\mathrm{X}, \Delta$ l'ensemble des couleurs de $\mathrm{X}$, et $\Delta\left(\mathrm{S}^{\prime}\right)$ la réunion des $\Delta(\alpha), \alpha \in \mathrm{S}^{\prime}$.

Proposition 3.4 - Pour qu'il existe un G-morphisme $\phi: \mathrm{X} \rightarrow \mathrm{G} / \mathrm{G}_{-\mathrm{S}^{\prime}}$ provenant d'une induction parabolique, il faut et il suffit que $\mathrm{S}^{\prime} \supset \operatorname{supp}(\Sigma) \cup \mathrm{S}^{p}$. Ce morphisme est alors unique, et est donné par $\Delta(\phi)=\Delta\left(\mathrm{S}^{\prime}\right)$.

Preuve - Soit $\phi: \mathrm{X} \rightarrow \mathrm{G} / \mathrm{Q}$ un G-morphisme provenant d'une induction parabolique, où $\mathrm{Q}=\mathrm{G}_{-\mathrm{S}^{\prime}}$. Du fait que $\mathrm{X}^{\prime}=\phi^{-1}(\mathrm{Q} / \mathrm{Q})$ est alors une $\mathrm{G}^{\prime}$-variété magnifique, et que $\mathrm{X}$ est isomorphe à $\mathrm{G} *_{\mathrm{Q}} \mathrm{X}^{\prime}$, on déduit sans peine que $\mathrm{S}^{\prime} \supset \operatorname{supp}(\Sigma) \cup \mathrm{S}^{p}$ et que $\phi$ est donné par $\Delta(\phi)=\Delta\left(\mathrm{S}^{\prime}\right)$.

Inversement, supposons $\mathrm{S}^{\prime} \supset \operatorname{supp}(\Sigma) \cup \mathrm{S}^{p}$. Alors $\Delta\left(\mathrm{S}^{\prime}\right)$ est visiblement un sousensemble distingué parabolique de $\Delta$, donc définit un G-morphisme $\phi: \mathrm{X} \rightarrow \mathrm{G} / \mathrm{Q}$ où $\mathrm{Q}=\mathrm{G}_{-\mathrm{S}^{\prime}}$. Reste à voir que $\mathrm{Q}^{r}$ opère trivialement dans $\mathrm{X}^{\prime}=\phi^{-1}(\mathrm{Q} / \mathrm{Q})$.

Pour tout $\Sigma^{\prime} \subset \Sigma$, notons $\mathrm{X}\left(\Sigma^{\prime}\right)$ la sous-variété magnifique de $\mathrm{X}$ dont les racines sphériques sont $\Sigma^{\prime}$, et posons $\mathrm{X}^{\prime}\left(\Sigma^{\prime}\right)=\mathrm{X}^{\prime} \cap \mathrm{X}\left(\Sigma^{\prime}\right)$. Montrons, par récurrence sur card $\left(\Sigma^{\prime}\right)$, que

(\#) $G^{\prime}$ n'a qu'une seule orbite fermée dans $\mathrm{X}^{\prime}\left(\Sigma^{\prime}\right)$.

De la classification des variétés magnifiques de rang 1 résulte que $\mathrm{Q}^{r}$ opère trivialement dans les $\mathrm{X}^{\prime}(\{\gamma\})(\gamma \in \Sigma)$, d'où (\#) dans ces cas. Supposons $\operatorname{card}\left(\Sigma^{\prime}\right) \geqslant 2$, et notons $\mathrm{X}^{\prime}\left(\Sigma^{\prime}\right)_{\mathrm{G}^{\prime}}^{\circ}$ l'orbite ouverte de $\mathrm{G}^{\prime}$ dans $\mathrm{X}^{\prime}\left(\Sigma^{\prime}\right)$. De l'hypothèse de récurrence on déduit que la composante connexe de $\mathrm{X}^{\prime}\left(\Sigma^{\prime}\right) \backslash \mathrm{X}^{\prime}\left(\Sigma^{\prime}\right)_{\mathrm{G}^{\prime}}^{\circ}$ contenant l'orbite fermée de Q, ne contient qu'une seule orbite fermée de $\mathrm{G}^{\prime}$. Mais si $\operatorname{card}\left(\Sigma^{\prime}\right) \geqslant 2, \mathrm{X}^{\prime}\left(\Sigma^{\prime}\right) \backslash \mathrm{X}^{\prime}\left(\Sigma^{\prime}\right)_{\mathrm{G}^{\prime}}^{\circ}$ est connexe (cela résulte par exemple de la théorie des «bouts» d'espaces topologiques), d'où (\#) pour $\Sigma^{\prime}$.

Du fait que $G^{\prime}$ n'a qu'une seule orbite fermée dans $\mathrm{X}^{\prime}$ résulte alors que $\mathrm{C}^{\prime}$, le centre connexe de $\mathrm{G}^{\prime}$, opère trivialement dans $\mathrm{X}^{\prime}$. Puisque la réunion des conjugués de $\mathrm{C}^{\prime}$ dans $\mathrm{Q}^{r}$ est dense dans $\mathrm{Q}^{r}$, il s'ensuit bien que $\mathrm{Q}^{\prime}$ opère trivialement dans $\mathrm{X}^{\prime}$.

Une variété magnifique irréductible de rang $\geqslant 1$, qui ne peut être obtenue par induction parabolique au moyen d'aucun sous-groupe parabolique propre, sera appelée cuspidale. La proposition précédente implique en particulier que, pour qu'une variété magnifique soit cuspidale, il faut et il suffit que son système sphérique soit cuspidal (c'est-à-dire vérifie $\operatorname{supp}(\Sigma)=\mathrm{S}$ ). 


\subsection{Produits fibrés}

Soient $\mathrm{X}, \mathrm{X}_{1}, \mathrm{X}_{2}$ et $\mathrm{X}^{\prime}$ quatre $\mathrm{G}$-variétés magnifiques, et soient $\psi_{1}: \mathrm{X} \rightarrow \mathrm{X}_{1}$, $\psi_{2}: \mathrm{X} \rightarrow \mathrm{X}_{2}, \phi_{1}: \mathrm{X}_{1} \rightarrow \mathrm{X}^{\prime}, \phi_{2}: \mathrm{X}_{2} \rightarrow \mathrm{X}^{\prime}$ quatre G-morphismes de variétés vérifiant $\phi_{1} \circ \psi_{1}=\phi_{2} \circ \psi_{2}$. Ces données impliquent un morphisme de G-variétés $\psi: \mathrm{X} \rightarrow$ $\mathrm{X}_{1} \times_{\mathrm{X}^{\prime}} \mathrm{X}_{2}$. En général, la G-variété $\mathrm{X}_{1} \times_{\mathrm{X}^{\prime}} \mathrm{X}_{2}$ n'est plus sphérique (sa dimension peut être $>\operatorname{dimB}$ !), et même si elle l'est, le morphisme $\psi$ n'est pas toujours un isomorphisme.

On devrait sans doute étudier systématiquement ces produits fibrés. Notre but ici est plus modeste, nous visons seulement un résultat particulier (la proposition 3.5), qui nous servira dans la démonstration du théorème 1 au $§ 5$.

Soit $\mathrm{S}^{p}, \Sigma, \mathbf{A}$ un système sphérique et soit $\Delta$ son ensemble de couleurs. Si $\Delta^{\prime}$ est un sous-ensemble distingué de $\Delta$, on pose $\mathrm{S}^{p}\left(\Delta^{\prime}\right)=\left(\mathrm{S}^{p} / \Delta^{\prime}\right) \backslash \mathrm{S}^{p}$ et $\Sigma\left(\Delta^{\prime}\right)=\Sigma \backslash\left(\Sigma / \Delta^{\prime}\right)$.

Soient $\Delta_{1}$ et $\Delta_{2}$ deux sous-ensembles distingués de $\Delta$. Alors le sous-ensemble $\Delta^{\prime}=\Delta_{1} \cup \Delta_{2}$ est encore distingué dans $\Delta$. On dira que le couple $\Delta_{1}, \Delta_{2}$ décompose le système sphérique $\mathrm{S}^{p}, \Sigma, \mathbf{A}$ (en produit fibré), si

(1) $\Delta_{1} \neq \varnothing, \Delta_{2} \neq \varnothing$ et $\Delta_{1} \cap \Delta_{2}=\varnothing$;

(2) $\Delta_{1}, \Delta_{2}$ et $\Delta^{\prime}$ possèdent la propriété $\left(^{*}\right)$;

(3) $\Sigma\left(\Delta_{1}\right) \cap \Sigma\left(\Delta_{2}\right)=\varnothing$;

(4) $\mathrm{S}^{p}\left(\Delta_{1}\right)$ est orthogonal à $\mathrm{S}^{p}\left(\Delta_{2}\right)$;

(5) $\Delta_{1}$ ou $\Delta_{2}$ est lisse.

Proposition 3.5 - Soit $\mathrm{S}^{p}, \Sigma, \mathbf{A}$ un système sphérique. On suppose donné un couple $\Delta_{1}, \Delta_{2}$ (de sous-ensembles distingués de $\Delta$ ) qui décompose $\mathrm{S}^{p}, \Sigma, \mathbf{A}$. On suppose aussi qu'il existe trois variétés magnifiques $\mathrm{X}_{1}, \mathrm{X}_{2}$ et $\mathrm{X}^{\prime}$, uniques à isomorphisme près, ayant comme systèmes sphériques $\left(\mathrm{S}^{p}, \Sigma, \mathbf{A}\right) / \Delta_{1},\left(\mathrm{~S}^{p}, \Sigma, \mathbf{A}\right) / \Delta_{2}$ et $\left(\mathrm{S}^{p}, \Sigma, \mathbf{A}\right) / \Delta^{\prime}$. Alors il existe des $G$-morphismes, uniques à isomorphisme près, $\phi_{1}: \mathrm{X}_{1} \rightarrow \mathrm{X}^{\prime}$ et $\phi_{2}: \mathrm{X}_{2} \rightarrow \mathrm{X}^{\prime}$ vérifiant : $\Sigma, \mathbf{A}$;

1) le produit fibré $\mathrm{X}_{1} \times_{\mathrm{X}^{\prime}} \mathrm{X}_{2}$ est une G-variété magnifique dont le système sphérique est $\mathrm{S}^{p}$,

2) si $\mathrm{X}$ est une $G$-variété magnifique dont le système sphérique est $\mathrm{S}^{p}, \Sigma, \mathbf{A}$, alors $\mathrm{X}$ est isomorphe à $\mathrm{X}_{1} \times \mathrm{X}^{\prime} \mathrm{X}_{2}$.

Preuve - Les ensembles de couleurs $\Delta_{\mathrm{X}_{i}}$ s'identifient aux ensembles $\Delta \backslash \Delta_{i}(i=1,2)$. Modulo ces identifications, $\Delta_{1}$ est distingué dans $\Delta_{\mathrm{X}_{2}}$, et $\Delta_{2}$ est distingué dans $\Delta_{\mathrm{X}_{1}}$, et les variétés quotient $\mathrm{X}_{2} / \Delta_{1}$ et $\mathrm{X}_{1} / \Delta_{2}$ ont comme système sphérique $\left(\mathrm{S}^{p}, \Sigma, \mathrm{A}\right) / \Delta^{\prime}$. De l'hypothèse d'unicité résulte que $\mathrm{X}_{2} / \Delta_{1}=\mathrm{X}^{\prime}=\mathrm{X}_{1} / \Delta_{2}$ (à isomorphisme près), et qu'on a des G-morphismes (uniques à isomorphisme près) $\phi_{1}: \mathrm{X}_{1} \rightarrow \mathrm{X}^{\prime}$ et $\phi_{2}: \mathrm{X}_{2} \rightarrow \mathrm{X}^{\prime}$.

Posons $\mathrm{X}_{12}=\mathrm{X}_{1} \times_{\mathrm{X}^{\prime}} \mathrm{X}_{2}$. De (5) résulte que $\mathrm{X}_{12}$ est lisse. Notons $\mathrm{Z}_{1}, \mathrm{Z}_{2}, \mathrm{Z}_{12}, \mathrm{Z}^{\prime}$ les orbites fermées de $\mathrm{G}$ et $z_{1}, z_{2}, z_{12}, z^{\prime}$ les points fixes de $\mathrm{B}_{-}$dans $\mathrm{X}_{1}, \mathrm{X}_{2}, \mathrm{X}_{12}, \mathrm{X}^{\prime}$. De (3) résulte que $Z_{12}$ est isomorphe à $Z_{1} \times_{Z^{\prime}} Z_{2}$. Supposons $\Delta_{1}$ lisse. Alors les vecteurs 
propres de $\mathrm{T}$ dans $\mathrm{T}_{z_{2}} \mathrm{X}_{2} / \mathrm{T}_{z_{2}} \mathrm{Z}_{2}$ qui s'envoient vers 0 dans $\mathrm{T}_{z^{\prime}} \mathrm{X}^{\prime} / \mathrm{T}_{z^{\prime}} \mathrm{Z}^{\prime}$ sont ceux des poids $\Sigma\left(\Delta_{1}\right)$. Les poids de $\mathrm{T}$ dans $\mathrm{T}_{z_{1}} \mathrm{X}_{1} / \mathrm{T}_{z_{1}} \mathrm{Z}_{1}$ sont $\Sigma \backslash \Sigma\left(\Delta_{1}\right)$. Par suite, les poids de $\mathrm{T}$ dans $\mathrm{T}_{z_{12}} \mathrm{X}_{12} / \mathrm{T}_{z_{12}} \mathrm{Z}_{12}$ sont $\Sigma$. Il s'ensuit que $\mathrm{X}_{12}$ est sphérique. Que $\mathrm{X}_{12}$ est magnifique résulte par exemple de la caractérisation suivante des variétés magnifiques : ce sont les variétés sphérique, lisses, complètes, ne contenant qu'une seule orbite fermée, et dont aucune couleur ne contient l'orbite fermée. Que $\mathrm{X}_{12}$ possède la dernière propriété vient de ce que toute couleur de $\mathrm{X}_{12}$ se projette sur l'un des facteurs au moins en couleur, et que les deux facteurs sont magnifiques. Cela montre que $\Sigma_{\mathrm{X}_{12}}=\Sigma$. Que $\mathrm{S}_{\mathrm{X}_{12}}^{p}=\mathrm{S}^{p}$ résulte de $\mathrm{Z}_{12} \cong \mathrm{Z}_{1} \times_{\mathrm{Z}^{\prime}} \mathrm{Z}_{2}$ et de (4). Enfin, que $\mathbf{A}_{\mathrm{X}_{12}}=\mathbf{A}$ peut se voir par exemple par localisation et réduction au rang 2 .

Soit maintenant $\mathrm{X}$ une G-variété magnifique dont le système sphérique est $\mathrm{S}^{p}$, $\Sigma$, A. Les ensembles $\Delta_{1}, \Delta_{2}$ fournissent des G-morphismes $\psi_{1}: \mathrm{X} \rightarrow \mathrm{X}_{1}, \psi_{2}: \mathrm{X} \rightarrow \mathrm{X}_{2}$ vérifiant $\phi_{1} \circ \psi_{1}=\phi_{2} \circ \psi_{2}$. D'où un G-morphisme $\psi: \mathrm{X} \rightarrow \mathrm{X}_{12}$. Des propriétés de $\mathrm{X}_{12}$ qu'on vient de voir résulte que $\psi: \mathrm{Z} \rightarrow \mathrm{Z}_{12}$ est un isomorphisme et que $\psi$ est étale en $z$. Cela implique que $\psi: \mathrm{X} \rightarrow \mathrm{X}_{12}$ est un isomorphisme.

Remarque - La proposition précédente s'applique en particulier aux factorisations (voir 3.1).

\subsection{Fibrations projectives}

Soit $\mathrm{S}^{p}, \Sigma, \mathbf{A}$ un système sphérique. Si $\delta \in \mathbf{A}$ vérifie $\delta(\Sigma) \subset\{0,1\}$, on dira que $\delta$ est un élément projectif de $\mathbf{A}$.

Soit $\delta$ un élément projectif de $\mathbf{A}$. Posons $\mathrm{S}_{\delta}=\delta^{-1}(1) \subset \mathrm{S} \cap \Sigma$. Alors $\{\delta\}$, considéré comme sous-ensemble de $\Delta$, est distingué lisse, et le triplet quotient $\left(\mathrm{S}^{p}, \Sigma, \mathbf{A}\right) /\{\delta\}$ est donné par :

$\mathrm{S}^{p} /\{\boldsymbol{\delta}\}=\mathrm{S}^{p}$

$\Sigma /\{\delta\}=\Sigma \backslash S_{\delta} ;$

$\mathbf{A} /\{\delta\}=$ la restriction de $\mathbf{A}\left(\mathrm{S} \backslash \mathrm{S}_{\delta}\right)$ au sous-groupe (de $\Xi$ ) engendré par $\Sigma /\{\delta\}$.

Si X est une G-variété magnifique dont le système sphérique est $\mathrm{S}^{p}, \Sigma, \mathbf{A}$, et si $\delta$ est un élément projectif de $\mathbf{A}$, on notera $\phi_{\delta}: \mathrm{X} \rightarrow \mathrm{X}_{\delta}$ le $\mathrm{G}$-morphisme correspondant à $\{\delta\}$.

Dans ce numéro, on verra que ce G-morphisme est une «fibration projective», au sens suivant : il est lisse, ses fibres sont toutes isomorphes à un espace projectif $\mathrm{P}_{n}$, et $\operatorname{rang}(\mathrm{X})=n+\operatorname{rang}\left(\mathrm{X}_{\delta}\right)$ (ces propriétés caractérisent probablement les morphismes $\phi_{\delta}$ ). Lorsque $\mathrm{G}$ est de type $\mathrm{A}$, on précisera la structure de $\phi_{\delta}$, puis on en déduira le résultat suivant (qui interviendra dans la preuve du théorème 1) :

Proposition $3.6-$ On suppose $G$ de type $A$. Soit $\mathrm{S}^{p}, \Sigma, \mathbf{A}$ un système sphérique et soit $\delta$ un élément projectif de $\mathbf{A}$. Soit $\mathrm{X}^{\prime}$ une $G$-variété magnifique dont le triplet est $\left(\mathrm{S}^{p}, \Sigma, \mathbf{A}\right) /\{\delta\}$. Alors il existe une $G$-variété magnifique $X$, unique à isomorphisme près, vérifiant : 
1) $\mathrm{S}^{p}, \Sigma, \mathbf{A}$ est le système sphérique de $X$;

2) $\mathrm{X}_{\delta}$ est G-isomorphe à $\mathrm{X}^{\prime}$.

3.6.1 Soit X une G-variété magnifique dont le système sphérique est $\mathrm{S}^{p}, \Sigma, \mathbf{A}$, et soit $\delta$ un élément projectif de $\mathbf{A}$. Notons $\mathrm{D}_{\delta}$ le diviseur de $\mathrm{X}$ correspondant à $\delta$.

a) Considérons d'abord le cas particulier $\mathrm{S}=\mathrm{S}_{\delta}=\Sigma$. Dans ce cas, $\mathrm{X}_{\delta}=\mathrm{G} / \mathrm{B}_{-}$, d'où il suit que $\mathrm{X}$ est $\mathrm{G}$-isomorphe à $\mathrm{G} *_{\mathrm{B}_{-}} \mathrm{Y}$, où $\mathrm{Y}=\phi_{\delta}^{-1}\left(\mathrm{~B}_{-} / \mathrm{B}_{-}\right)$. La variété $\mathrm{Y}$ est visiblement l'espace d'un plongement torique de $\mathrm{T}$.

Explicitons l'éventail associé à ce plongement (qui se trouve dans $\mathrm{N}_{\mathrm{T} /\{\ell\}}$, qu'on peut identifier à $N_{X}$ ). Les diviseurs irréductibles T-stables de $\mathrm{Y}$ sont les $\mathrm{D}^{\beta} \cap \mathrm{Y}$ $(\beta \in \Sigma=\mathrm{S})$ et $\mathrm{D}_{\delta} \cap \mathrm{Y}$. Les demi-droites associées à ces diviseurs sont : d'une part, les $\boldsymbol{Q}_{+}\left(-\beta^{*}\right)(\beta \in \mathrm{S})$ correspondant aux $\mathrm{D}^{\beta} \cap \mathrm{Y}$ (qui sont stables par $\mathrm{B}_{-}$); et, d'autre part, la demi-droite $\mathbf{Q}_{+}\left(\sum_{\alpha \in S} \alpha^{*}\right)$ correspondante à $D_{\delta} \cap \mathrm{Y}$ (qui n'est pas stable par $\mathrm{B}_{-}$). Notons $\mathrm{C}_{\mathrm{S}}$ le cône engendré par les $-\beta^{*},(\beta \in \mathrm{S})$, et $\mathrm{C}_{\mathrm{S}, \alpha}$ celui engendré par les $-\beta^{*}$, $(\beta \in S \backslash\{\alpha\})$ et par $\left.\sum_{\alpha \in S} \alpha^{*}\right)$. L'unique point fixe de $B_{-}$correspond au cône $\mathrm{C}_{\mathrm{S}}$. Partant de là, on voit que l'éventail est celui dont les cônes maximaux sont $\mathrm{C}_{\mathrm{S}}$ et les $\mathrm{C}_{\mathrm{S}, \alpha}$, $\alpha \in \mathrm{S}$.

Par suite, $\mathrm{Y}$ est isomorphe à l'espace projectif $\mathrm{P}_{m}$, où $m=$ cardS (et les diviseurs irréductibles stables par $\mathrm{T}$ de $\mathrm{Y}$ sont tous isomorphes à $\left.\mathrm{P}_{m-1}\right)$. Désignons par $\mathrm{A}_{\mathrm{Y}, \delta}$ le groupe des automorphismes de $\mathrm{Y}$ qui laissent stable chaque $\mathrm{D}^{\beta} \cap \mathrm{Y}(\beta \in \mathrm{S})$, et par $\mathrm{A}_{\mathrm{Y}, \delta}^{u}$ le radical unipotent de $\mathrm{A}_{\mathrm{Y}, \delta}$. D'après la théorie des «systèmes d'Enriques» (voir [De], p. 577, théorème 4), on a $\mathrm{A}_{\mathrm{Y}, \delta}=\mathrm{TA}_{\mathrm{Y}, \delta}^{u}$, le groupe $\mathrm{A}_{\mathrm{Y}, \delta}^{u}$ est commutatif, les poids de $\mathrm{T}$ dans $\operatorname{Lie}\left(\mathrm{A}_{\mathrm{Y}, \delta}^{u}\right)$ sont les $-\beta, \beta \in \mathrm{S}$, et chaque espace propre est de dimension 1 . L'opération de $\mathrm{B}_{-}$dans $\mathrm{Y}$ est donnée par un morphisme (de groupes algébriques) $\mathrm{B}_{-} \rightarrow \mathrm{A}_{\mathrm{Y}, \delta}$ qui est surjectif et qui prolonge l'opération de T. Un morphisme avec ces propriétés est clairement unique, à conjugaison par un élément de $\mathrm{T}$ près.

b) Revenons au cas général.

L'ensemble $\{\delta\}$ étant distingué lisse, le morphisme $\phi_{\delta}: \mathrm{X} \rightarrow \mathrm{X}_{\delta}$ est lisse (voir 3.3). Notons $X^{\delta}$ le localisé de $X$ en $S_{\delta}$. Posons $L_{\delta}=G_{\mathrm{S}_{\delta}} \cap G_{-S_{\delta}}$. Le localisé de $X_{\delta}$ en $S_{\delta}$ est visiblement $\mathrm{L}_{\delta} \cdot z_{\delta}$ (où $z_{\delta}$ est l'unique point fixe de $\mathrm{B}_{-}$dans $\mathrm{X}_{\delta}$ ), d'où $\phi_{\delta}\left(\mathrm{X}^{\delta}\right)=\mathrm{L}_{\delta} \cdot z_{\delta}$. De 3.2 , on déduit que la $\mathrm{L}_{\delta}$-variété $\mathrm{X}^{\delta}$ vérifie les hypothèses de $a$ ), donc que $\mathrm{Y}^{\delta}=\left(\left.\phi_{\delta}\right|_{\mathrm{X}^{\delta}}\right)^{-1}\left(z_{\delta}\right)$ est isomorphe à l'espace projectif $\mathrm{P}_{m}$, où $m=\operatorname{card} \mathrm{S}_{\delta}=\operatorname{dim} \mathrm{X}-\operatorname{dim} \mathrm{X}_{\delta}$. Puisque $\phi_{\delta}$ est lisse et à fibres connexes, on en déduit que $\mathrm{Y}^{\delta}=\phi_{\delta}^{-1}\left(z_{\delta}\right)$, et que chaque fibre de $\phi_{\delta}$ est isomorphe à $\mathrm{P}_{m}$. 
3.6.2 Conservons les hypothèses de 3.6.1. Posons $\Delta=\Delta_{X}, \Xi=\Xi_{X}$ et $\rho=\rho_{X}$ : $\Delta \rightarrow \Xi^{*}$.

L'ensemble $\Delta\left(\mathrm{S} \backslash \mathrm{S}_{\delta}\right) \cup\left\{\mathrm{D}_{\delta}\right\}$ est visiblement un sous-ensemble distingué parabolique de $\Delta$, donc fournit un $\mathrm{G}$-morphisme $\psi: \mathrm{X} \rightarrow \mathrm{G} / \mathrm{Q}$ où $\mathrm{Q}=\mathrm{G}_{-\left(\mathrm{S} / \mathrm{s}_{\delta}\right)}$. Désignons par $\mathrm{L}$ le sous-groupe de Levi de $\mathrm{Q}$ contenant T. Notons $\psi_{\delta}: \mathrm{X}_{\delta} \rightarrow \mathrm{G} / \mathrm{Q}$ le G-morphisme tel que $\psi=\psi_{\delta} \circ \phi_{\delta}$. Alors $\mathrm{X}_{\delta} \cong \mathrm{G} *_{\mathrm{Q}} \mathrm{Y}_{\delta}$, où $\mathrm{Y}_{\delta}=\phi_{\delta}^{-1}(\mathrm{Q} / \mathrm{Q})$.

Lemme - Si $\mathrm{G}$ est de type $\mathrm{A}$, alors $\mathrm{Q}^{r}$ (le radical de $\mathrm{Q}$ ) opère trivialement dans $\mathrm{Y}_{\delta}$ (donc $\mathrm{Y}_{\delta}$ est une $\mathrm{L}$-variété magnifique, et $\mathrm{X}_{\delta}$ est obtenu par induction parabolique à travers $\mathrm{Q}$ au moyen de $\left.\mathrm{Y}_{\delta}\right)$.

Preuve - Puisque $\Sigma_{\mathrm{X}_{\delta}}=\Sigma \backslash \mathrm{S}_{\delta}$, d'après 3.4 on doit montrer que $\mathrm{S}_{\delta} \cap \operatorname{supp}\left(\Sigma \backslash \mathrm{S}_{\delta}\right)=\varnothing$. Par localisation, il suffit de le vérifier lorsque $\mathrm{X}$ est de rang 2, ce qui est facile (voir la liste dans 4.1).

Remarque - L'assertion du lemme n'est pas vraie en général. Par exemple, supposons $G$ de type $B_{2}$, et notons $\alpha_{1}, \alpha_{2}$ ses deux racines simples ( $\alpha_{1}$ étant la racine longue). Considérons le système sphérique $S^{p}=\varnothing, \Sigma=\left\{\alpha_{1}+\alpha_{2}, \alpha_{2}\right\}$ et $\mathbf{A}=\mathbf{A}\left(\alpha_{2}\right)$ composé de deux éléments projectifs. Si $\delta$ est l'un d'eux, alors $\left(\mathbf{S}^{p}, \Sigma, \mathbf{A}\right) /\{\delta\}=\left(\varnothing,\left\{\alpha_{1}+\right.\right.$ $\left.\left.\alpha_{2}\right\}, \varnothing\right)$, système sphérique qui est cuspidal.

Lorsque $\mathrm{G}$ est de type $\mathrm{A}$, nous allons montrer que la Q-variété $\mathrm{Y}$ et le morphisme $\phi_{\delta}: \mathrm{Y} \rightarrow \mathrm{Y}_{\delta}$ ne dépendent que de la $\mathrm{G}$-variété $\mathrm{X}_{\delta}$ et du triplet $\mathrm{S}^{p}, \Sigma, \mathbf{A}$. Commençons par considérer la L-variété (sphérique) $\mathrm{Y}$ (et le L-morphisme $\phi_{\delta}: \mathrm{Y} \rightarrow \mathrm{Y}_{\delta}$ ).

\section{La variété $\mathrm{Y}$ comme L-variété}

Choisissons des sous-groupes $\mathrm{H}$ et $\mathrm{H}_{\delta}$ de $\mathrm{L}$ vérifiant $\mathrm{Y}_{\mathrm{L}}^{\circ} \cong \mathrm{L} / \mathrm{H},\left(\mathrm{Y}_{\delta}\right)_{\mathrm{L}}^{\circ} \cong \mathrm{L} / \mathrm{H}$ et $\mathrm{H} \subset \mathrm{H}_{\delta}$. Il est clair que $\mathrm{H}_{\delta}$ est la clôture sphérique de $\mathrm{H}$ (voir §6). On peut identifier $\mathrm{S}_{\mathrm{L} / \mathrm{H}}^{p}=\mathrm{S}_{\mathrm{L} / \mathrm{H}_{\delta}}^{p}$ à $\mathrm{S}^{p}, \Delta_{\mathrm{L} / \mathrm{H}}=\Delta_{\mathrm{L} / \mathrm{H}_{\delta}}$ à $\Delta\left(\mathrm{S} \backslash \mathrm{S}_{\delta}\right), \Sigma_{\mathrm{L} / \mathrm{H}}=\Sigma_{\mathrm{L} / \mathrm{H}_{\delta}}$ à $\Sigma \backslash \mathrm{S}_{\delta}, \Xi_{\mathrm{L} / \mathrm{H}}$ à $\Xi$, $\Xi_{\mathrm{L} / \mathrm{H}_{\delta}}$ au sous-groupe de $\Xi$ engendré par $\Sigma \backslash \mathrm{S}_{\delta}$ (qu'on peut noter $\Xi_{\delta}$ ), et modulo ces identifications, $\rho_{\mathrm{G} / \mathrm{H}}$ est égal à la restriction de $\rho$. On sait que $\mathrm{H}$ est déterminé par $\mathrm{H}_{\delta}$ et par le couple $\Xi_{\mathrm{L} / \mathrm{H}}, \rho_{\mathrm{G} / \mathrm{H}}$ (voir §6). Par suite $\mathrm{H}$ est déterminé par $\mathrm{X}_{\delta}$ et le triplet $\mathrm{S}^{p}, \Sigma, \mathbf{A}$.

Pour décrire la L-variété $\mathrm{Y}$ et le L-morphisme $\phi_{\delta}: \mathrm{Y} \rightarrow \mathrm{Y}_{\delta}$ (en termes de $\mathrm{X}_{\delta}$ et du triplet $\left.\mathrm{S}^{p}, \Sigma, \mathbf{A}\right)$, il reste à expliciter l'éventail colorié associé au plongement de $\mathrm{L} / \mathrm{H}$ dans $\mathrm{Y}$ (éventail qui se trouve dans $\operatorname{Hom}_{\mathbf{Z}}(\boldsymbol{\Xi}, \mathbf{Q})$ ).

Les diviseurs irréductibles L-stables de $\mathrm{Y}$ sont les $\mathrm{D}^{\beta} \cap \mathrm{Y}(\beta \in \Sigma)$ et $\mathrm{D}_{\delta} \cap \mathrm{Y}$. Les demi-droites associées à ces diviseurs sont : d'une part, les $\mathbf{Q}_{+}\left(-\beta^{*}\right)(\beta \in \Sigma)$ correspondant aux $\mathrm{D}^{\beta} \cap \mathrm{Y}$ (diviseurs qui sont stables par $\mathrm{Q}$ ); et, d'autre part, la 
demi-droite $\mathbf{Q}_{+}\left(\sum_{\alpha \in S_{\delta}} \alpha^{*}\right)$ correspondante à $D_{\delta} \cap \mathrm{Y}$ (qui n'est pas stable par $\mathbf{Q}$ ). Partant de là, on voit, comme dans 3.6.1 a), que l'éventail colorié en question est celui, sans couleurs, dont les cônes maximaux sont $\mathrm{C}_{\Sigma}$ et les $\mathrm{C}_{\Sigma, \alpha}, \alpha \in \mathrm{S}_{\delta}$ (où l'on a noté $\mathrm{C}_{\Sigma}$ le cône engendré par les $-\gamma^{*},(\gamma \in \Sigma)$, et $\mathrm{C}_{\Sigma, \alpha}$ celui engendré par les $-\gamma^{*},(\gamma \in \Sigma \backslash\{\alpha\})$ et $\left.\sum_{\alpha \in \mathrm{S}_{\delta}} \alpha^{*}\right)$.

\section{L'opération de $\mathrm{Q}^{u}$ dans $\mathrm{Y}$}

Désignons par $\mathrm{A}_{\mathrm{Y}, \delta}^{*}$ le groupe des automorphismes unipotents de $\mathrm{Y}$ qui laissent stables chaque fibre de $\phi_{\delta}: \mathrm{Y} \rightarrow \mathrm{Y}_{\delta}$ et chaque $\mathrm{D}^{\beta} \cap \mathrm{Y}(\beta \in \Sigma)$. Par définition, on a un morphisme naturel $\theta^{*}: \mathrm{A}_{\mathrm{Y}, \delta}^{*} \rightarrow \mathrm{A}_{\mathrm{Y}^{\delta}, \delta}^{u}$. Le groupe $\mathrm{L}$ opère de façon naturelle dans $\mathrm{A}_{\mathrm{Y}, \delta}^{*}$. L'opération de $\mathrm{Q}^{u}$ dans $\mathrm{Y}$ est donnée par un morphisme $\theta: \mathrm{Q}^{u} \rightarrow \mathrm{A}_{\mathrm{Y}, \delta}^{*}$ compatible avec les opérations de $\mathrm{L}$ et tel que $\theta^{*} \circ \theta: \mathrm{Q}^{u} \rightarrow \mathrm{A}_{\mathrm{Y}^{\delta}, \delta}^{u}$ est surjectif.

3.6.3 Démontrons maintenant la proposition 3.6.

\section{Unicité}

Nous avons déjà expliqué pourquoi la L-variété $\mathrm{Y}$ et le morphisme $\phi_{\delta}: \mathrm{Y} \rightarrow \mathrm{Y}_{\delta}$ sont déterminés par la $\mathrm{G}$-variété $\mathrm{X}_{\delta}$ et le triplet $\mathrm{S}^{p}, \Sigma, \mathbf{A}$.

D'après 3.6.1 a), $\mathrm{A}_{\mathrm{Y}, \delta}^{*}$ est commutatif, et les poids de $\mathrm{G}$ dans $\operatorname{Lie}\left(\mathrm{A}_{\mathrm{Y}, \delta}^{*}\right)$ sont les $-\left.\beta\right|_{\mathrm{C}}, \beta \in \mathrm{S}_{\delta}$. Vu la structure du morphisme $\phi_{\delta}: \mathrm{Y} \rightarrow \mathrm{Y}_{\delta}$, et vu la théorie des «systèmes d'Enriques» de [De], il existe un fibré en droites $\mathbf{L}^{\beta}$ sur $\mathrm{Y}_{\delta}$ tel que les champs de vecteurs de $\operatorname{Lie}\left(\mathrm{A}_{\mathrm{Y}, \delta}^{*}\right)$ de poids $-\left.\beta\right|_{\mathrm{C}}$ sous $\mathrm{C}$, peuvent être interprétés comme sections globales de $\mathbf{L}^{\beta}$. Puisque $Y_{\delta}$ est sphérique sous $L$, deux sections de $\mathbf{L}^{\beta}$ ayant un poids $-\beta$ sous $L \cap B$ ne diffèrent que d'un scalaire multiplicatif. Le L-module $\operatorname{Lie}\left(\mathrm{Q}^{u}\right) /\left[\operatorname{Lie}\left(\mathrm{Q}^{u}\right), \operatorname{Lie}\left(\mathrm{Q}^{u}\right)\right]$ étant isomorphe à la somme directe des L-modules simples de poids dominant $-\beta, \beta \in \mathrm{S}_{\beta}$, il s'ensuit que deux morphismes $\theta: \mathrm{Q}^{u} \rightarrow \mathrm{A}_{\mathrm{Y}, \delta}^{*}$, compatibles avec les opérations de $\mathrm{L}$ et tels que $\theta^{*} \circ \theta: \mathrm{Q}^{u} \rightarrow \mathrm{A}_{\mathrm{Y}^{\delta}, \delta}^{u}$ est surjectif, sont conjugués par un élément de $\mathrm{C}$.

On en déduit que la Q-variété $\mathrm{Y}$ (à isomorphisme près) est déterminée par la $\mathrm{G}$-variété $\mathrm{X}_{\delta}$ et le triplet $\mathrm{S}^{p}, \Sigma, \mathbf{A}$. Puisque $\mathrm{X}$ est $\mathrm{G}$-isomorphe à $\mathrm{G} *_{\mathrm{Q}} \mathrm{Y}$, il en est de même pour la G-variété X.

\section{Existence}

Soient $\mathrm{X}^{\prime}$ et $\mathrm{S}^{p}, \Sigma, \mathbf{A}$ comme dans la proposition 3.6. On a $\mathrm{X}^{\prime}=\mathrm{G} *_{\mathrm{Q}} \mathrm{Y}^{\prime}$, où $\mathrm{Y}^{\prime}$ est une L-variété magnifique. Posons $\mathrm{L} / \mathrm{H}^{\prime}=\left(\mathrm{Y}^{\prime}\right)_{\mathrm{L}}^{\circ}$. On commence par construire $\mathrm{H}$ comme le sous-groupe de $\mathrm{L}$ de clôture sphérique $\mathrm{H}^{\prime}$, associé au couple $\Xi_{\mathrm{L} / \mathrm{H}}=\Xi$, $\rho_{\mathrm{G} / \mathrm{H}}=$ la restriction de $\rho$ à $\Delta\left(\mathrm{S} \backslash \mathrm{S}_{\delta}\right)$ (voir $\left.\S 6\right)$.

Puis on construit la L-variété $\mathrm{Y}$ (comme le plongement de $\mathrm{L} / \mathrm{H}$ associé à l'éventail colorié introduit ci-dessus, voir 3.6.2), avec un L-morphisme $\phi^{\prime}: \mathrm{Y} \rightarrow \mathrm{Y}^{\prime}$. Cela permet en particulier d'introduire les fibrés en droites $\mathbf{L}^{\beta}, \beta \in \mathrm{S}_{\delta}$. 
Montrons que le poids $-\beta$ apparaît effectivement comme poids de $\mathrm{L} \cap \mathrm{B}$ dans $\mathrm{H}^{0}\left(\mathrm{Y}^{\prime}, \mathbf{L}^{\beta}\right)$. Soit

$$
\left[\mathbf{L}^{\beta}\right]=\sum_{\mathrm{D} \in \Delta_{\mathrm{Y}^{\prime}}} n(\beta, \mathrm{D})[\mathrm{D}]
$$

l'expression de $\left[\mathrm{L}^{\beta}\right]$ dans la base $[\mathrm{D}], \mathrm{D} \in \Delta_{\mathrm{Y}^{\prime}}$, de $\operatorname{Pic}\left(\mathrm{Y}^{\prime}\right)$.

\section{Lemme}

1) Si $\alpha \in \mathrm{S} \backslash \mathrm{S}_{\delta}$ est tel que $<\boldsymbol{\alpha}^{\mathrm{v}}, \boldsymbol{\beta}>=0$, alors $n(\boldsymbol{\beta}, \mathrm{D})=0$, quel que soit $\mathrm{D} \in \Delta_{\mathrm{Y}^{\prime}}(\boldsymbol{\alpha})$.

2) Si $\alpha \in \mathrm{S} \backslash \mathrm{S}_{\delta}$ est tel que $<\alpha^{\mathrm{v}}, \beta>\neq 0$, alors :

ou bien $\alpha$ est de type a, et $\left\{n\left(\beta, \mathrm{D}_{\alpha}^{+}\right), n\left(\beta, \mathrm{D}_{\alpha}^{-}\right)\right\}=\left\{-<\alpha^{\mathrm{v}}, \beta>, 0\right\}$;

ou bien $\alpha$ est de type b, et $n\left(\beta, \mathrm{D}_{\alpha}\right)=-\left\langle\alpha^{\mathrm{v}}, \beta>\right.$.

Preuve - Par «localisation», on se ramène au cas où $S_{\delta}=\{\beta\}$ et $S=\{\alpha, \beta\}$, auquel cas les assertions du lemme se vérifient facilement.

Reprenons la preuve de la proposition. Rappelons que pour un groupe semisimple $\mathrm{G}$, et un diviseur B-stable effectif $d$ sur une G-variété sphérique (lisse) $\mathrm{X}, \sigma_{\mathrm{X}}(d)$ désigne le poids de la section canonique de $\mathbf{L}_{d}$ (le fibré en droites associé à $d$ ).

$$
\begin{array}{ll}
\text { Posons } & d=\sum_{\mathrm{D} \in \Delta_{\mathrm{Y}^{\prime}}} n(\beta, \mathrm{D}) \mathrm{D} \\
& \Delta(\beta)=\left\{\mathrm{D} \in \Delta_{\mathrm{Y}^{\prime}}, \quad n(\beta, \mathrm{D}) \neq 0\right\} \text { et } \\
& \mathrm{S}(\boldsymbol{\beta})=\left\{\alpha \in \mathrm{S} \backslash \mathrm{S}_{\delta}, \quad<\alpha^{\mathrm{v}}, \boldsymbol{\beta}>0\right\} .
\end{array}
$$

Le lemme montre que $d$ est effectif, que pour tout $\mathrm{D} \in \Delta(\beta)$ il existe un unique $\alpha(\mathrm{D}) \in \mathrm{S}(\beta)$ tel que $\mathrm{D} \in \Delta_{\mathrm{Y}^{\prime}}(\alpha(\mathrm{D}))$, qu'on obtient ainsi une bijection de $\Delta(\beta)$ sur $\mathrm{S}(\beta)$, et que $\sigma_{Y^{\prime}}(\mathrm{D})=\omega_{\alpha(\mathrm{D})}$. Par suite

$$
\sigma_{Y^{\prime}}(d)=\sum_{\alpha \in S^{\prime} S_{\delta}}-<\alpha^{v}, \beta>\omega_{\alpha}=-\beta,
$$

où l'on considère ici $-\beta$ comme poids de $[\mathrm{L}, \mathrm{L}] \cap \mathrm{B}$. Puisque $\mathbf{L}_{d}$ est isomorphe à $\mathbf{L}^{\beta}$, il s'ensuit bien que $-\beta$ se trouve parmi les poids de $\mathrm{L} \cap \mathrm{B}$ dans $\mathrm{H}^{0}\left(\mathrm{Y}^{\prime}, \mathbf{L}^{\beta}\right)$.

Comme la section canonique de $d$ ne s'annule pas au point $z^{\prime}$ (l'unique point fixe de $\mathrm{B}_{-} \cap \mathrm{L}$ dans $\left.\mathrm{Y}^{\prime}\right)$, et vu la structure de $\operatorname{Lie}\left(\mathrm{Q}^{u}\right) /\left[\operatorname{Lie}\left(\mathrm{Q}^{u}\right), \operatorname{Lie}\left(\mathrm{Q}^{u}\right)\right]$, on déduit de ce qui précède l'existence d'un morphisme $\theta: \mathrm{Q}^{u} \rightarrow \mathrm{A}_{\mathrm{Y}, \delta}^{*}$, compatible avec les opérations de $\mathrm{L}$ et tel que $\theta^{*} \circ \theta: \mathrm{Q}^{u} \rightarrow \mathrm{A}_{\mathrm{Y}^{\delta}, \delta}^{u}$ est surjectif (où $\mathrm{Y}^{*}$ est ici $\left(\phi^{\prime}\right)^{-1}\left(z^{\prime}\right)$ ). D'où l'opération de $\mathrm{Q}$ dans $\mathrm{Y}$.

Par construction, $\mathrm{X}=\mathrm{G} *_{\mathrm{Q}} \mathrm{Y}$ possède alors les propriétés voulues. 


\section{§4 Étude combinatoire des systèmes sphériques de type A}

Dans tout ce $\S$, sauf mention expresse du contraire, $\mathrm{S}$ désignera la base d'un système de racines de type $\mathrm{A}$, et tous les systèmes sphériques considérés seront supposés «adjoints» (c'est-à-dire composés de racines sphériques du réseau radiciel).

\subsection{Diagrammes}

Le premier but visé dans ce $\S$ est une représentation «graphique» des systèmes sphériques, sous forme de «diagrammes» (en partant du diagramme de Dynkin de G).

Soit $\mathrm{S}^{p}, \Sigma, \mathbf{A}$ un système sphérique. Rappelons qu'il induit une décomposition de $\mathrm{S}$ en quatre sous-ensembles : $\mathrm{S}^{p}, \mathrm{~S}^{a}=\mathrm{S} \cap \Sigma, \mathrm{S}^{a^{\prime}}=\mathrm{S} \cap \frac{1}{2} \Sigma$, et $\mathrm{S}^{b}=\mathrm{S} \backslash\left(\mathrm{S}^{a} \cup \mathrm{S}^{a^{\prime}} \cup \mathrm{S}^{p}\right)$.

Comme première information sur le système sphérique, on représente «graphiquement» cette décomposition : selon que $\alpha$ est dans $\mathrm{S}^{a}$ (resp. dans $\mathrm{S}^{a^{\prime}}, \mathrm{S}^{b}$ ou $\mathrm{S}^{p}$ ), on ajoute au sommet correspondant à $\alpha$ (du diagramme de Dynkin) le dessin suivant :

$$
\text { genre } a \stackrel{\bigcirc}{\ominus} \text { genre } a^{\prime} \quad \dot{\bigcirc} \text { genre } b \quad \odot \text { genre } p \text {. }
$$

Rappelons qu'au système sphérique est associé un ensemble de couleurs $\Delta$ qui, par construction, est réunion d'ensembles $\Delta(\alpha), \alpha \in \mathrm{S}$ (en général non disjoints) contenant 2, 1 ou 0 élément. On voit que le nombre de «ronds» qu'on vient d'ajouter à chaque sommet $\alpha$ correspond au nombre des couleurs de $\Delta(\alpha)$. Pour représenter «graphiquement» les couleurs, comme deuxième information portée sur le diagramme de Dynkin, on reliera par un trait fin pointillé ceux des ronds qui correspondent à une même couleur.

Les informations portées jusqu'ici sur le diagramme de Dynkin permettent déjà de connaître les racines sphériques de type $a_{1}$, $a^{\prime}$ et $a_{1} \times a_{1}$. Voici comment on va représenter les autres racines sphériques $\gamma(\operatorname{lorsque} \mathrm{S}=\operatorname{supp}(\gamma))$ :

$$
\begin{aligned}
& \text { type } a_{1}: \stackrel{\bigcirc}{\odot} \text { type } a_{n}(n>1): \odot ـ \ldots \\
& \text { type } a^{\prime}: \dot{\bigcirc} \text { type } d_{3}: \bullet \text { type } \mathrm{a}_{1} \times a_{1}: \odot
\end{aligned}
$$

Reste à représenter «graphiquement» la famille $\mathbf{A}$. Lorsque $\alpha \in \mathrm{S} \cap \Sigma$, rappelons que $\mathbf{A}(\alpha)=\left\{\delta_{\alpha}^{+}, \delta_{\alpha}^{-}\right\}=\{\delta \in \mathbf{A}, \delta(\alpha)=1\}$. On convient que $\delta_{\delta}^{+}$correspond au rond supérieur associé au sommet $\alpha$ (du diagramme de Dynkin), et $\delta_{\alpha}^{-}$au rond inférieur.

On s'arrange pour que $\left.<\delta_{\alpha}^{+}, \gamma\right\rangle \in\{1,0,-1\}$ quel que soit $\gamma \in \Sigma \backslash\{\alpha\}$ (cela est toujours possible, quitte à échanger $\delta_{\alpha}^{+}$et $\delta_{\alpha}^{-}$. Il ne reste alors plus qu'une ambiguïté, qu'on lève de la façon suivante : 
si $\gamma \in \Sigma \backslash\{\alpha\}$ est tel que $\left\langle\alpha^{v}, \gamma>\neq 0\right.$ et si $\left\langle\delta_{\alpha}^{+}, \gamma\right\rangle=-1$, alors on ajoute une «flèche» $>($ ou $<)$ à côté du rond correspondant à $\delta_{\alpha}^{+}$, et «pointant vers» $\gamma$

(dans tous les autres cas, on retrouve les $\mathbf{A}(\alpha)$ par les informations dont on dispose déjà, grâce aux propriétés de $\mathbf{A}$ ).

Remarque — La règle pour l'emploi des «flèches» peut paraître à première vue compliquée, mais se révèle simple en pratique. À titre d'exemple, considérons les cas suivants :
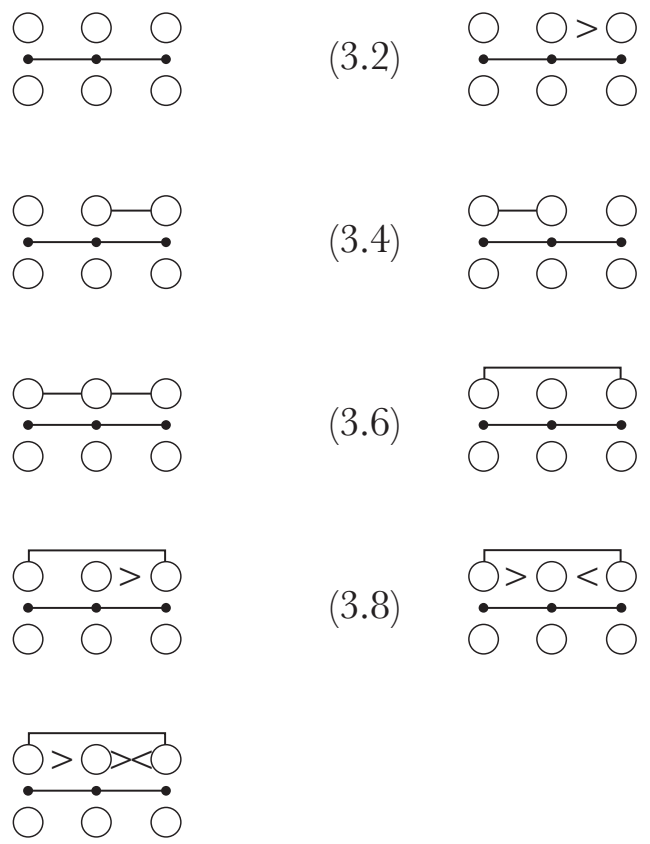

Ce sont tous les systèmes sphériques dans $A_{3}$ vérifiant $S^{p}=\varnothing$ et $\Sigma=S=\left\{\alpha_{1}, \alpha_{2}, \alpha_{3}\right\}$. Concentrons-nous plus particulièrement sur les cas (3.6)-(3.9). Dans ces cas, A comprend cinq éléments $\delta_{\alpha_{1}}^{+}=\delta_{\alpha_{3}}^{+}, \delta_{\alpha_{1}}^{-}, \delta_{\alpha_{2}}^{+}, \delta_{\alpha_{2}}^{-}, \delta_{\alpha_{3}}^{-}$, dont les valeurs sur $\alpha_{1}, \alpha_{2}$, $\alpha_{3}$ sont données par les matrices suivantes :

$\begin{array}{rrrrrrrrrrrr}1 & 0 & 1 & 1 & 0 & 1 & 1 & -1 & 1 & 1 & -1 & 1 \\ 1 & -1 & -1 & 1 & -1 & -1 & 1 & 0 & -1 & 1 & 0 & -1 \\ 0 & 1 & 0 & 0 & 1 & -1 & 0 & 1 & 0 & 0 & 1 & -1 \\ -1 & 1 & -1 & -1 & 1 & 0 & -1 & 1 & -1 & -1 & 1 & 0 \\ -1 & -1 & 1 & -1 & -1 & 1 & -1 & 0 & 1 & -1 & 0 & 1\end{array}$

Pour un autre exemple d'emploi de «flèches», voir le cas $3^{\prime}$ ) plus bas.

Voici les «diagrammes» de tous les systèmes sphériques (adjoints, de type A) de rang 2 cuspidaux et non factorisables (en deux facteurs de rang 1). 


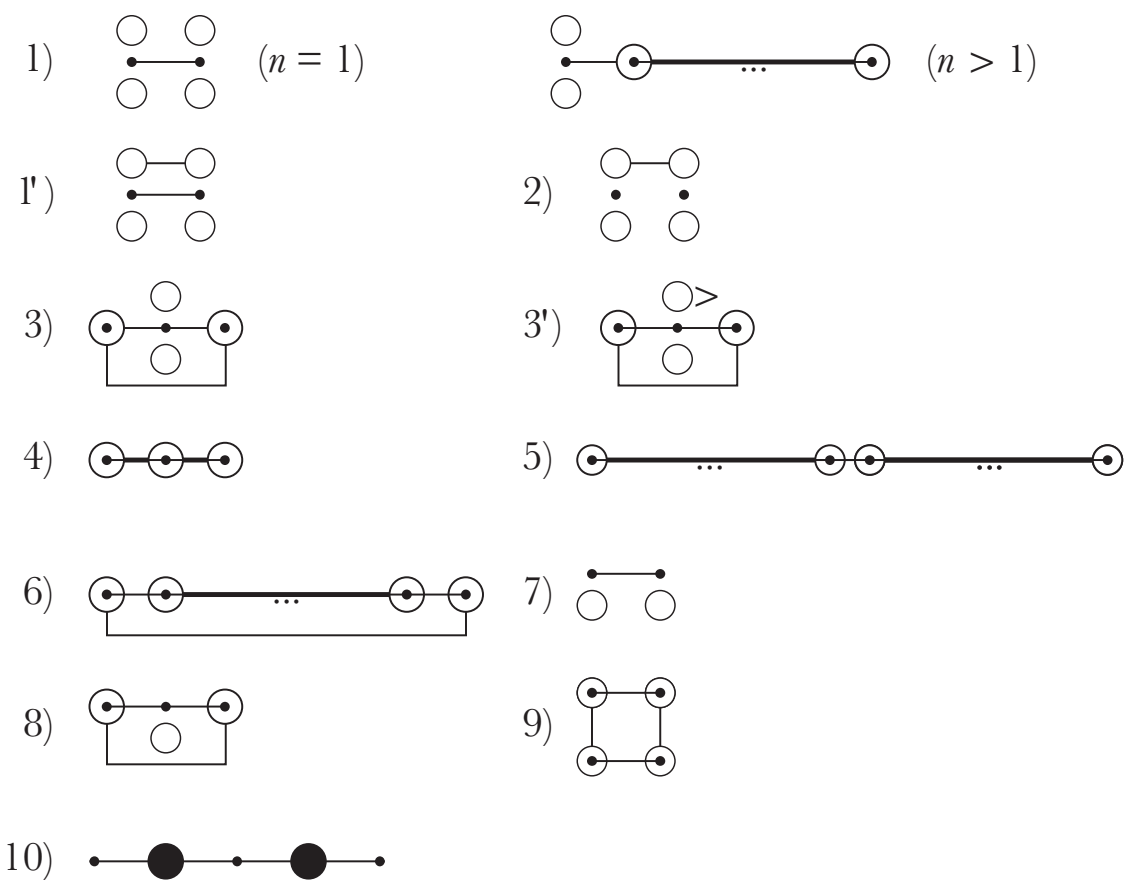

Remarques - 1) Cette liste découle des axiomes des systèmes sphériques. Elle apparaît aussi déjà, sous une forme légèrement différente, dans [Wa] (comme conséquence de la classification des variétés magnifiques de rang 2), voir p. 382, table A.

2) Comme visiblement (par définition) un système $S^{p}, \Sigma, \mathbf{A}$ est sphérique si et seulement si ses sous-systèmes (au sens de 3.1 ) de rang $\leqslant 2$ le sont, la liste ci-dessus peut «remplacer» les axiomes des systèmes sphériques. Plus visuelle, elle permet souvent de mieux raisonner que les axiomes. Par exemple, il suffit de la considérer un peu pour constater que les systèmes sphériques $\mathrm{S}^{p}, \Sigma, \mathbf{A}$ de type $\mathrm{A}$ possèdent les propriétés suivantes :

(1) quel que soit $\gamma \in \Sigma$, le système obtenu par localisation en $S^{\prime}=\operatorname{supp}(\gamma)$ est toujours de rang 1 ;

(2) quel que soit le couple $\gamma_{1}, \gamma_{2} \in \Sigma, \gamma_{1} \neq \gamma_{2}$ le système obtenu par localisation en $S^{\prime}=\operatorname{supp}\left(\gamma_{1}\right) \cup \operatorname{supp}\left(\gamma_{2}\right)$ est toujours de rang 2 (excepté si $\alpha_{1}+\alpha_{2}, \alpha_{2}+\alpha_{3}, \alpha_{3}+\alpha_{4} \in \Sigma$, où $\alpha_{1}, \alpha_{2}, \alpha_{3}, \alpha_{4}$ sont des éléments consécutifs de $S$, et si $\gamma_{1}=\alpha_{1}+\alpha_{2}, \gamma_{2}=\alpha_{3}+\alpha_{4}$ ).

Ces propriétés ne sont plus vraies pour les systèmes de type $B$.

L'intérêt des «diagrammes» vient de ce qu'ils rendent plus concret tout ce qui concerne les systèmes sphériques (comme les notions du $\S 3$ : localisation, quotient, induction parabolique, produit fibré, fibration projective). On en verra de nombreux exemples dans la suite de ce paragraphe et le suivant. En voici dès à présent quelquesuns. 
A) Soit $\mathbf{S}^{p}, \Sigma, \mathbf{A}$ le système sphérique suivant (déjà rencontré ci-dessus) :

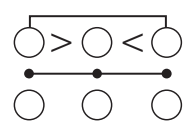

L'ensemble $\Delta$ des couleurs de ce système contient cinq sous-ensembles distingués non paraboliques $\Delta^{\prime}$ :

$$
\begin{aligned}
& \left\{\delta_{\alpha_{2}}^{+}\right\},\left\{\delta_{\alpha_{1}}^{-}, \delta_{\alpha_{3}}^{-}\right\},\left\{\delta_{\alpha_{1}}^{+}, \delta_{\alpha_{2}}^{-}\right\} \\
& \left\{\delta_{\alpha_{1}}^{-}, \delta_{\alpha_{3}}^{-}, \delta_{\alpha_{2}}^{+}\right\},\left\{\delta_{\alpha_{1}}^{+}, \delta_{\alpha_{1}}^{-}, \delta_{\alpha_{2}}^{-}, \delta_{\alpha_{3}}^{-}\right\},
\end{aligned}
$$

dont les systèmes sphériques quotients $\left(\mathrm{S}^{p}, \Sigma, \mathbf{A}\right) / \Delta^{\prime}$ sont :
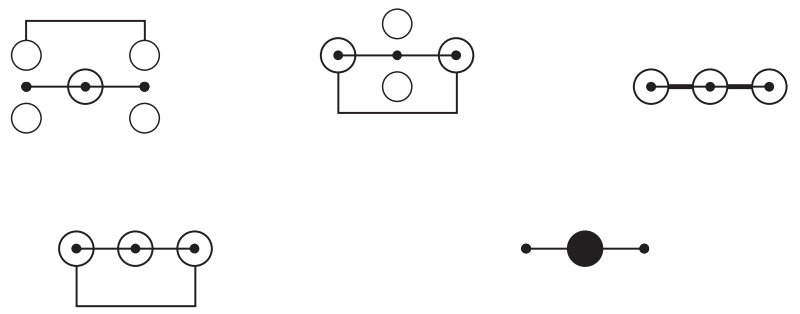

Parmi les cinq systèmes sphériques ci-dessus, lesquels sont cuspidaux?

B) Voici un exemple simple de «produit fibré» (au sens de 3.5) :

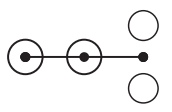

est produit

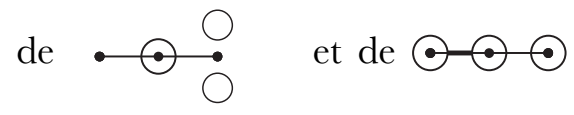

au-dessus de $\bullet \odot$

(on a $\Delta_{1}=\left\{\delta_{\alpha_{1}}\right\}$ et $\Delta_{2}=\left\{\delta_{\alpha_{3}}^{+}\right\}$).

G) En passant, nous avons rencontré aussi des «fibrations projectives» (au sens de 3.6) : par exemple pour $\mathrm{S}^{p}, \Sigma, \mathbf{A}$ égal à

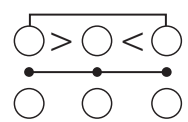

$\delta=\delta_{\alpha_{2}}^{+}$est un élément projectif de $\mathbf{A}$, et $\left(\mathrm{S}^{p}, \Sigma, \mathbf{A}\right) /\{\delta\}$ est

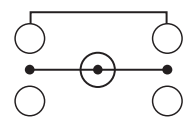


4.2 La liste des cas «primitifs»

Dans ce qui suit, on donnera une liste d'exemples de systèmes sphériques, qu'on appellera primitifs. L'intérêt des systèmes primitifs pour la structure générale des systèmes sphériques sera expliqué dans les numéros suivants. Ils jouent un rôle important dans la preuve du théorème 1 au $\S 5$.

On notera $\mathrm{S}=\left\{\alpha_{1}, \ldots, \alpha_{n}\right\}$ la base usuelle du système de racines de type $\mathrm{A}_{n}$, $n \geqslant 1$. La notation $\left\{\alpha_{p}, \ldots, \alpha_{q}\right\}$ désignera, lorsque $p \leqslant q$, l'ensemble des racines simples $\alpha_{i}(p \leqslant i \leqslant q)$, et lorsque $p>q$, l'ensemble vide.

(1) La famille $a o(n), n \geqslant 1$ :

$$
\begin{aligned}
& \mathrm{S}^{p}=\varnothing \\
& \Sigma=\left\{2 \alpha_{1}, \ldots, 2 \alpha_{n}\right\} \\
& \mathbf{A}=\varnothing
\end{aligned}
$$

Diagramme de $a o(7)$ :

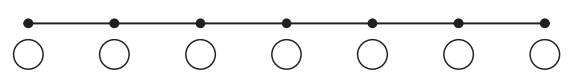

(2) La famille $a c(n), n$ impair $\geqslant 3$ :

$$
\begin{aligned}
& \mathrm{S}^{p}=\left\{\alpha_{i}, i \text { impair, } 1 \leqslant i \leqslant n\right\} \\
& \Sigma=\left\{\alpha_{1}+2 \alpha_{2}+\alpha_{3}, \alpha_{3}+2 \alpha_{4}+\alpha_{5}, \ldots, \alpha_{n-2}+2 \alpha_{n-1}+\alpha_{n}\right\} \\
& \mathbf{A}=\varnothing
\end{aligned}
$$

Diagramme de $a c(7)$ :

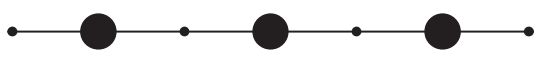

Les familles $a a$

D'abord,

(3) la famille $a a(p+q+p), n=2 p+q, p \geqslant 1, q \geqslant 1$ :

$$
\begin{aligned}
& \mathrm{S}^{p}=\left\{\alpha_{p+2}, \ldots, \alpha_{p+q-1}\right\} \\
& \Sigma=\left\{\alpha_{1}+\alpha_{n}, \alpha_{2}+\alpha_{n-1}, \ldots, \alpha_{p}+\alpha_{n-p+1}, \alpha_{p+1}+\ldots+\alpha_{p+q}\right\} \text { si } q \geqslant 2 \\
& \text { (si } \left.q=1, \Sigma=\left\{\alpha_{1}+\alpha_{n}, \alpha_{2}+\alpha_{n-1}, \ldots, \alpha_{p}+\alpha_{n-p+1}, \alpha_{p+1}\right\}\right) \\
& \mathbf{A}=\varnothing \text { si } q \geqslant 2 \text { (si } q=1, \mathbf{A} \text { est l'unique famille adaptée à } \Sigma, \\
& \text { sans élément projectif). }
\end{aligned}
$$

Diagramme de $a a(2+3+2)$ :

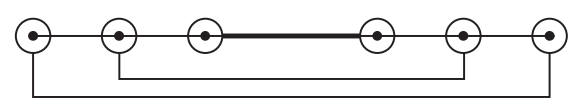


Puis les familles (4) $a a(p, p)$ et (5) $a a(q)$ obtenues par localisation de $a a(p+q+p)$ en

$$
\begin{aligned}
& \mathrm{S}^{\prime}=\left\{\alpha_{1}, \ldots, \alpha_{p}, \alpha_{p+q+1}, \ldots, \alpha_{n}\right\} \text { et } \\
& \mathrm{S}^{\prime}=\left\{\alpha_{p+1}, \ldots, \alpha_{p+q}\right\} .
\end{aligned}
$$

Enfin,

(6) la famille $a a^{*}(p+1+p), p \geqslant 1$ :

$$
\begin{aligned}
& \mathrm{S}^{p}=\varnothing \\
& \Sigma=\left\{\alpha_{1}+\alpha_{n}, \alpha_{2}+\alpha_{n-1}, \ldots, \alpha_{p}+\alpha_{n-p+1}+2 \alpha_{p+1}\right\} \\
& \mathbf{A}=\varnothing
\end{aligned}
$$

Diagramme de $a a^{*}(3+1+3)$ :

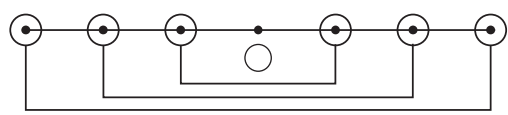

Les familles ci-dessus s'appellent les « cas classiques ». Il y a ensuite quatre autres familles, les « cas supplémentaires ».

(7) La famille $a c^{*}(n), n \geqslant 3$ :

$$
\begin{aligned}
& S^{p}=\varnothing \\
& \Sigma=\left\{\alpha_{1}+\alpha_{2}, \alpha_{2}+\alpha_{3}, \ldots, \alpha_{n-1}+\alpha_{n}\right\} \\
& \mathbf{A}=\varnothing
\end{aligned}
$$

Diagramme de $a c^{*}(7)$ :

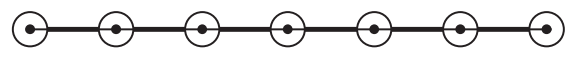

Les familles $a x$

D'abord,

(8) la famille $\operatorname{ax}(1+p+1+q+1), n=p+q+3, p \geqslant 1, q \geqslant 1$ :

$$
\begin{aligned}
& \mathrm{S}^{p}=\left\{\alpha_{3}, \ldots, \alpha_{p}, \alpha_{p+4}, \ldots, \alpha_{p+q+1}\right\} \\
& \Sigma=\left\{\alpha_{1}, \alpha_{2}+\ldots+\alpha_{p+1}, \alpha_{p+2}, \alpha_{p+3}+\ldots+\alpha_{p+q+2}, \alpha_{n}\right\}
\end{aligned}
$$

$\mathbf{A}$ est l'unique famille adaptée à $\Sigma$, sans élément projectif, contenant un élément qui prend les valeurs $\begin{array}{lllllllll}1 & -1 & 1 & 0 & -1 & \text { sur } & \alpha_{1}, & \alpha_{2}+\ldots+\end{array}$ $\alpha_{p+1}, \alpha_{p+2}, \alpha_{p+3}+\ldots+\alpha_{p+q+2}, \alpha_{n}$.

Diagramme de $a x(1+3+1+2+1)$ :

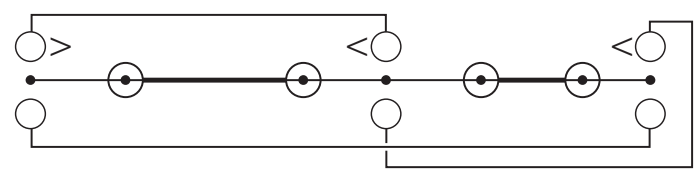


Puis les familles (9) $a x(1+p+1,1)$ et (10) $a x(1+p+1)$, et le cas isolé (11) $a x(1,1,1)$, obtenus de $a x(1+p+1+q+1)$ par localisation en

$$
\begin{aligned}
& \mathrm{S}^{\prime}=\left\{\alpha_{1}, \ldots, \alpha_{p+2}, \alpha_{n}\right\}, \\
& \mathrm{S}^{\prime}=\left\{\alpha_{1}, \ldots, \alpha_{p+2}\right\} \text { et } \\
& \mathrm{S}^{\prime}=\left\{\alpha_{1}, \alpha_{p+2}, \alpha_{n}\right\} .
\end{aligned}
$$

Les familles ay

D'abord,

(12) la famille $a y(p+q+p), n=2 p+q, p \geqslant 2, q \geqslant 1$ :

$$
\begin{aligned}
& \mathrm{S}^{p}=\left\{\alpha_{p+2}, \ldots, \alpha_{p+q-1}\right\} \\
& \Sigma=\left\{\alpha_{1}, \ldots, \alpha_{p}, \alpha_{p+1}+\ldots+\alpha_{p+q}, \alpha_{p+q+1}, \ldots, \alpha_{n}\right\}
\end{aligned}
$$

A est l'unique famille adaptée à $\Sigma$, sans élément projectif, contenant un élément qui prend les valeurs 1 et -1 sur $\alpha_{n}$ et $\alpha_{1}$, et qui est nul ailleurs.

Diagramme de $a y(3+2+3)$ :

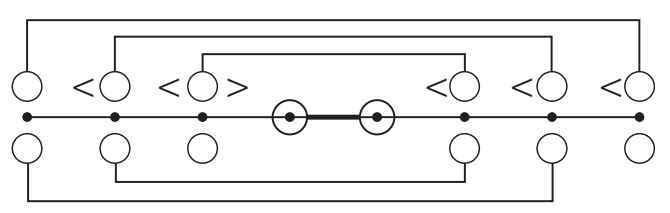

Puis les familles (13) ay $(p+q+p-1)$, (14) ay $(p, p)$, et (15) ay $(p, p-1)$, obtenues de $a y(p+q+p)$ par localisation en

$$
\begin{aligned}
& S^{\prime}=\left\{\alpha_{1}, \ldots, \alpha_{n-1}\right\} \\
& S^{\prime}=\left\{\alpha_{1}, \ldots, \alpha_{p}, \alpha_{p+q+1}, \ldots, \alpha_{n}\right\}, \text { et } \\
& S^{\prime}=\left\{\alpha_{1}, \ldots, \alpha_{p}, \alpha_{p+q+1}, \ldots, \alpha_{n-1}\right\} .
\end{aligned}
$$

Ensuite,

(16) la famille $a y^{\sim}(p+q+p), n=2 p+q, p \geqslant 2, q \geqslant 1$ :

$$
\begin{aligned}
& \mathrm{S}^{p}=\left\{\alpha_{p+2}, \ldots, \alpha_{p+q-1}\right\} \\
& \Sigma=\left\{\alpha_{1}, \ldots, \alpha_{p}, \alpha_{p+1}+\ldots+\alpha_{p+q}, \alpha_{p+q+1}, \ldots, \alpha_{n}\right\}
\end{aligned}
$$

$\mathbf{A}$ est l'unique famille adaptée à $\Sigma$, sans élément projectif, contenant un élément qui prend les valeurs 1 et -1 sur $\alpha_{p}$ et $\alpha_{n}$, et qui est nul ailleurs.

Diagramme de $a y^{\sim}(3+2+3)$ :

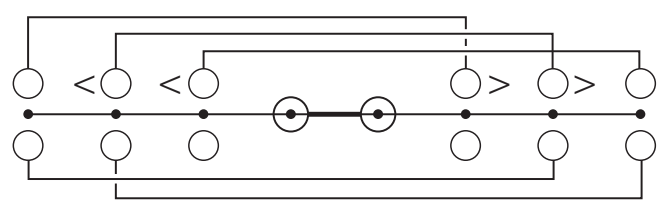


Enfin, (17) la famille $a y^{*}(2+q+2), n=4+q, q \geqslant 1$ :

$$
\begin{aligned}
& \mathrm{S}^{p}=\left\{\alpha_{4}, \ldots, \alpha_{q+1}\right\} \\
& \Sigma=\left\{\alpha_{1}, \alpha_{2}, \alpha_{3}+\ldots+\alpha_{q+2}, \alpha_{n-1}, \alpha_{n}\right\}
\end{aligned}
$$

A est l'unique famille adaptée à $\Sigma$, sans élément projectif, contenant un élément qui prend les valeurs 1 et -1 sur $\alpha_{1}$ et $\alpha_{n-1}$, et qui est nul ailleurs.

Diagramme de $a y^{*}(2+2+2)$ :

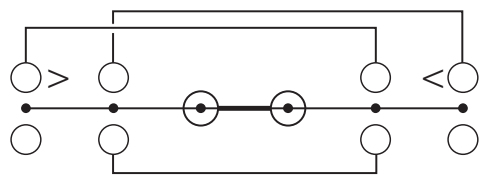

Les familles $a z$

D'abord (18) la famille $a z^{\sim}(3+q+3), n=6+q, q \geqslant 1$ :

$$
\begin{aligned}
& \mathrm{S}^{p}=\left\{\alpha_{5}, \ldots, \alpha_{2+q}\right\} \\
& \Sigma=\left\{\alpha_{1}, \alpha_{2}, \alpha_{3}, \alpha_{4}+\ldots+\alpha_{q+3}, \alpha_{n-2}, \alpha_{n-1}, \alpha_{n}\right\}
\end{aligned}
$$

A est l'unique famille adaptée à $\Sigma$, sans élément projectif, contenant un élément qui prend les valeurs $1,-1,-1$ sur $\alpha_{1}, \alpha_{3}, \alpha_{n-1}$, et qui est nul ailleurs.

Diagramme de $a z^{\sim}(3+1+3)$ :

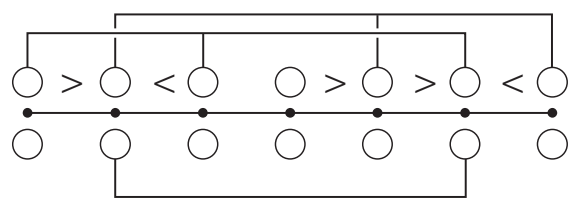

Puis (19) la famille $a z^{\sim}(3+q+2)$ et les cas isolés (20) $a z(3,3),(21) a z(3,2)$ et (22) $a z(3,1)$ obtenus en localisant $a z^{\sim}(3+q+3)$ en

$$
\begin{aligned}
& S^{\prime}=\left\{\alpha_{1}, \ldots, \alpha_{n-1}\right\}, \\
& S^{\prime}=\left\{\alpha_{1}, \alpha_{2}, \alpha_{3}, \alpha_{n-2}, \alpha_{n-1}, \alpha_{n}\right\} \\
& S^{\prime}=\left\{\alpha_{1}, \alpha_{2}, \alpha_{3}, \alpha_{n-2}, \alpha_{n-1}\right\} \text { et } \\
& S^{\prime}=\left\{\alpha_{1}, \alpha_{2}, \alpha_{3}, \alpha_{n-1}\right\} .
\end{aligned}
$$

Enfin, il y a quelques cas « exceptionnels».

Les cas $a e_{6}$

D'abord le cas (23) $a e_{6}(6), n=6$ :

$$
\begin{aligned}
& \mathrm{S}^{p}=\varnothing \\
& \Sigma=\left\{\alpha_{1}, \ldots, \alpha_{6}\right\}
\end{aligned}
$$


A est l'unique famille adaptée à $\Sigma$, sans élément projectif, contenant un élément qui prend les valeurs $1,-1,-1$ sur $\alpha_{1}, \alpha_{3}, \alpha_{5}$ et qui est nul ailleurs.

Diagramme de $a e_{6}(6)$ :

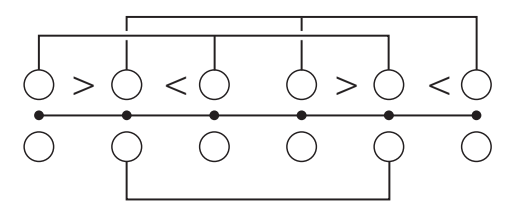

Deux autres cas (24) $a e_{6}(5)$ et (25) $a e_{6}(4)$ sont obtenus en localisant $a e_{6}(6)$ en

$$
\begin{aligned}
& S^{\prime}=\left\{\alpha_{1}, \ldots, \alpha_{5}\right\} \text { et } \\
& S^{\prime}=\left\{\alpha_{2}, \ldots, \alpha_{5}\right\} .
\end{aligned}
$$

Les cas $a e_{7}$

D'abord le cas (26) $a e_{7}(7), n=7$ :

$$
\begin{aligned}
& S^{p}=\varnothing \\
& \Sigma=\left\{\alpha_{1}, \ldots, \alpha_{7}\right\}
\end{aligned}
$$

A est l'unique famille adaptée à $\Sigma$, sans élément projectif, contenant un élément qui prend les valeurs 1 et -1 sur $\alpha_{1}$ et $\alpha_{5}$, et qui est nul ailleurs.

Diagramme de $a e_{7}(7)$ :

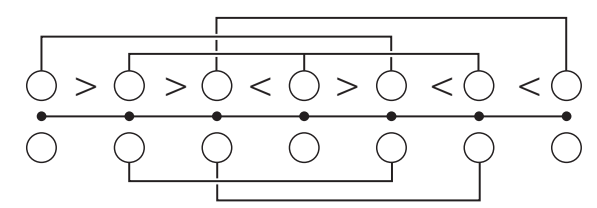

Puis deux autres cas (27) $a e_{7}(6)$ et (28) $a e_{7}(5)$ sont obtenus en localisant $a e_{7}(7)$ en

$$
\begin{aligned}
& S^{\prime}=\left\{\alpha_{1}, \ldots, \alpha_{6}\right\} \text { et } \\
& S^{\prime}=\left\{\alpha_{2}, \ldots, \alpha_{6}\right\} .
\end{aligned}
$$

(29) Le cas af(4), $n=4$ :

$$
\begin{aligned}
& \mathrm{S}^{p}=\varnothing \\
& \Sigma=\left\{\alpha_{1}, \ldots, \alpha_{4}\right\}
\end{aligned}
$$

A est donné par :

$$
\begin{array}{rrrr}
1 & -1 & 1 & 0 \\
1 & 0 & -1 & 0 \\
-1 & 0 & 1 & -1 \\
0 & 1 & -1 & 1 \\
0 & -1 & 0 & 1 \\
-1 & 1 & 0 & -1
\end{array}
$$


Diagramme de af(4) :

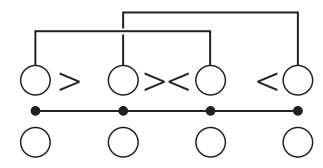

En résumé, il y a donc 29 cas primitifs. D’abord les 6 cas classiques (1) à (6) :

$$
\begin{aligned}
& a o(n)(n \geqslant 1) ; \\
& a c(n)(n \text { impair } \geqslant 3) ; \\
& a a(p+q+p), a a(p, p), a a(q), \quad a a^{*}(p+1+p)(p \geqslant 1, q \geqslant 1) ;
\end{aligned}
$$

puis les 16 cas supplémentaires (7) à (22) :

$$
\begin{aligned}
& a c^{*}(n)(n \geqslant 3) ; \\
& a x(1+p+1+q+1), a x(1+p+1,1), \quad a x(1+p+1), \quad a x(1,1,1) \\
& (p \geqslant 1, q \geqslant 1) ; \\
& a y(p+q+p), a y(p+q+p-1), \quad a y(p, p), \quad a y(p, p-1), \\
& a y \sim(p+q+p), a y^{*}(2+q+2)(p \geqslant 2, q \geqslant 1) ; \\
& a z^{\sim}(3+q+3), a z^{\sim}(3+q+2), a z(3,3), a z(3,2), a z(3,1)(q \geqslant 1) ;
\end{aligned}
$$

et enfin les 7 cas exceptionnels (23) à (29) :

$$
\begin{aligned}
& a e_{6}(6), a e_{6}(5), \quad a e_{6}(4) \text {; } \\
& a e_{7}(7), \quad a e_{7}(6), \quad a e_{7}(5) \text {; } \\
& \text { af }(4) \text {. }
\end{aligned}
$$

\subsection{La $\Delta$-connexité}

Soit $\mathrm{S}^{p}, \Sigma, \mathbf{A}$ un système sphérique, et soit $\Delta$ son ensemble de couleurs. Pour tout $\gamma \in \Sigma$, on notera $\Delta(\gamma)$ la réunion des $\Delta(\alpha), \alpha \in \operatorname{supp}(\gamma)$.

On dira que deux éléments $\gamma_{1}, \gamma_{2} \in \Sigma$ sont fortement $\Delta$-voisins si pour tout $\mathrm{D} \in \Delta\left(\gamma_{1}\right)$ on a $<\rho(D), \gamma_{2}>\neq 0$ et pour tout $\mathrm{D} \in \Delta\left(\gamma_{2}\right)$ on a $<\rho(\mathrm{D}), \gamma_{1}>\neq 0$.

On dira que $\gamma_{1}, \gamma_{2} \in \Sigma$ sont $\Delta$-voisins si :

ou bien ils le sont fortement;

ou bien il existe $\gamma_{3} \in \Sigma$ tel que le système obtenu par localisation en $\operatorname{supp}\left(\left\{\gamma_{1}, \gamma_{2}, \gamma_{3}\right\}\right)$ est isomorphe à $a x(1+q+1), q \geqslant 1$.

Un sous-ensemble $\Sigma^{\prime} \subset \Sigma$ sera dit $\Delta$-connexe (resp. fortement $\Delta$-connexe) si deux éléments quelconques dans $\Sigma^{\prime}$ peuvent être joints par une suite d'éléments de $\Sigma^{\prime}$, dont les éléments successifs sont $\Delta$-voisins (resp. fortement $\Delta$-voisins). Une composante $\Delta$-connexe de $\Sigma$ est un sous-ensemble $\Delta$-connexe maximal.

Les systèmes sphériques primitifs sont $\Delta$-connexes, sauf les cas ay $\sim(p+q+p)$, $a z^{\sim}(3+q+3)$ et $a z^{\sim}(3+q+2)$. 
Proposition 4.3 - Tout système sphérique $\Delta$-connexe cuspidal et sans couleur projective est primitif.

Preuve - Soit $\mathrm{S}^{p}, \Sigma, \mathbf{A}$ un système sphérique $\Delta$-connexe cuspidal et sans couleur projective.

Un regard sur la liste des systèmes sphériques de rang 2 montre que seule une racine sphérique de type $d_{3}$ peut être $\Delta$-voisine d'une racine sphérique de même type. Par suite, si $\Sigma$ contient une racine sphérique de type $d_{3}$, alors $\mathrm{S}^{p}, \Sigma$, $\mathbf{A}$ est isomorphe à $a c(n), n$ impair $\geqslant 3$.

On voit de même, si $\Sigma$ contient une racine sphérique de type $a^{\prime}$, alors $\mathrm{S}^{p}, \Sigma$, A est isomorphe à $a o(n), n \geqslant 1$ ou à $a a^{*}(p+1+p), p \geqslant 1$, et si $\Sigma$ contient une racine sphérique de type $a_{1} \times a_{1}$, alors $\mathrm{S}^{p}, \Sigma, \mathbf{A}$ est isomorphe à $a a(p+q+p), p \geqslant 1, q \geqslant 1$ ou à $a a(p, p), p \geqslant 1$ ou à $a a^{*}(p+1+p), p \geqslant 1$.

Il reste à examiner les cas où toutes les racines sphériques de $\Sigma$ sont de type $a_{n}$, $n \geqslant 1$. Si toutes les racines sphériques de $\Sigma$ sont de type $a_{n}, n \geqslant 2$, alors un regard sur la liste des cas de rang 2 montre que $\mathrm{S}^{p}, \Sigma, \mathbf{A}$ est isomorphe à $a a(q), q \geqslant 2$ ou à $a c^{*}(n)$, $n \geqslant 3$.

Lemme - Soit $\mathrm{S}^{p}, \Sigma, \mathbf{A}$ un système sphérique fortement $\Delta$-connexe, cuspidal, sans couleur projective. On suppose que toutes les racines sphériques sont de type $a_{n}(n \geqslant 1)$, qu'il existe une racine de type $a_{1}$, et que $\Sigma$ contient au moins deux éléments. Alors toutes les racines sphériques sont de type $a_{1}$, et le système est isomorphe (à automorphisme extérieur près) à l'un des 12 cas suivants :

$$
\begin{aligned}
& a x(1,1,1) ; \\
& a e_{6}(6), \quad a e_{6}(5), \quad a e_{6}(4) ; \\
& a e_{7}(7), \quad a e_{7}(6), \quad a e_{7}(5) ; \\
& a y(p, p), \quad a y(p, p-1)(p \geqslant 2) ; \\
& a z(3,3), \quad a z(3,2), \quad a z(3,1) .
\end{aligned}
$$

Preuve du lemme - Aucune racine sphérique de type $a_{n}(n \geqslant 2)$ ne peut être fortement $\Delta$-voisine d'une racine de type $a_{1}$, d'après la liste des cas de rang 2 , d'où la première asserttion du lemme. Il s'ensuit $\Sigma=\mathrm{S}$. La forte $\Delta$-connexité signifie alors que deux éléments de $\mathrm{S}$ peuvent être joints par une suite d'éléments dont deux successifs $\alpha, \beta$ vérifient : $\mathbf{A}(\alpha) \cap \mathbf{A}(\boldsymbol{\beta}) \neq \varnothing$. Vu les propriétés de $\mathbf{A}$, il en résulte que $\mathbf{A}$ (et donc le système sphérique) est déterminé dès qu'on connaît l'un de ses éléments.

L'ensemble $\mathrm{S}$ se décompose en « composantes connexes » dont on en notera $\left\{\alpha_{1}, \ldots, \alpha_{r}\right\}$ une de longueur maximale, puis $\left\{\beta_{1}, \ldots, \beta_{s}\right\}$ une deuxième, etc. Si $r=1$ (c'est-à-dire si les racines de $\mathrm{S}$ sont orthogonales deux à deux), alors les hypothèses sur $\mathbf{A}$ (forte $\Delta$-connexité et absence d'éléments projectifs), impliquent que le système sphérique est isomorphe à $a x(1,1,1)$.

Supposons donc $r \geqslant 2$. Dans ce cas, les hypothèses sur $\mathbf{A}$ impliquent qu'il existe un $\delta \in \mathbf{A}$ tel que $\delta\left(\alpha_{1}\right)=1$ et $\delta\left(\alpha_{2}\right)=0$. Ces mêmes hypothèses impliquent aussi qu'il 
existe un seul $\alpha \in S \backslash\left\{\alpha_{1}, \alpha_{2}, \alpha_{3}\right\}$ tel que $\delta(\alpha)=-1$, et si $\beta \in S \backslash\left\{\alpha_{1}, \alpha_{2}, \alpha_{3}\right\}$ est tel que $\delta(\beta)=1$, alors $\beta$ n'est pas orthogonal à $\alpha$. En particulier $S$ contient au plus deux composantes connexes. Les seules possibilités pour $\delta$ sont les suivantes (les autres, ou bien contredisent les propriétés de $\mathbf{A}$, ou bien ne produisent pas un système fortement $\Delta$-connexe).

$\mathrm{Ou}$ bien $\mathrm{S}$ est connexe, et

1) $r=7$ et $\delta$ prend les valeurs $1000-100$, auquel cas on trouve $a e_{7}(7)$;

2) $r=6$ et $\delta$ prend les valeurs $1000-10$ (ou les valeurs $10-11-10$ ), auquel cas on trouve $a e_{7}(6)$;

3) $r=6$ et $\delta$ prend les valeurs $10-10-10$, auquel cas on trouve $a e_{6}(6)$;

4) $r=5$ et $\delta$ prend les valeurs $10-11-1$, auquel cas on trouve $a e_{7}(5)$;

5) $r=5$ et $\delta$ prend les valeurs $10-10-1$ (ou les valeurs $101-11$ ), auquel cas on trouve $a e_{6}(5)$;

6) $r=4$ et $\delta$ prend les valeurs $101-1$, auquel cas on trouve $a e_{6}(4)$.

$\mathrm{Ou}$ bien $\mathrm{S}$ possède deux composantes connexes, et

1) $r=s=p \geqslant 2$ et $\delta$ prend les valeurs $10 \ldots 0$ et $-10 \ldots 0$ (ou les valeurs $10 \ldots 0$ et $1-10 \ldots 0)$, auquel cas on trouve ay $(p, p)$;

2) $r=p \geqslant 2, s=p-1$ et $\delta$ prend les valeurs $10 \ldots 0$ et $-10 \ldots 0$, auquel cas on trouve $a y(p, p-1)$;

3) $r=s=3$ et $\delta$ prend les valeurs $10-1$ et $0-10$, auquel cas on trouve $a z(3,3)$;

4) $r=3, s=2$ et $\delta$ prend les valeurs $10-1$ et $0-1$, auquel cas on trouve $a z(3,2)$;

5) $r=3, s=1$ et $\delta$ prend les valeurs $10-1$ et -1 , auquel cas on trouve $a z(3,1)$.

Revenons à la preuve de la proposition. Considérons le cas qui reste, c'est-àdire celui d'un système sphérique non isomorphe à $a a(1), \Delta$-connexe non fortement $\Delta$-connexe, cuspidal et sans couleur projective, dont toutes les racines sphériques sont de type $a_{n}(n \geqslant 1)$, l'une au moins étant de type $a_{1}$.

Pour obtenir ces systèmes, on doit « agrandir » ou " recoller » les exemples du lemme, en se servant de $a x(1+q+1)(q \geqslant 1)$. Il y a bien sûr $\operatorname{ax}(1+q+1)(q \geqslant 1)$ lui-même. Par " agrandissement ", on obtient $a x(1+p+1,1), a x(1+p+1+q+1)$, $(p \geqslant 1, q \geqslant 1)$, et ay $(p+q+p)$, ay $(p+q+p-1), a y^{*}(2+q+2)(p \geqslant 2, q \geqslant 1)$. Enfin aff $(4)$ est obtenu en « recollant » deux exemplaires de $a x(1+1+1)$. Il n'y a pas d'autres possibilités.

\subsection{Composantes $\Delta$-connexes effaçables}

Soit $\mathrm{S}^{p}, \Sigma, \mathbf{A}$ un système sphérique, et soit $\Sigma^{\prime}$ une composante $\Delta$-connexe de $\Sigma$.

Notons $\Delta\left(\Sigma^{\prime}\right)$ l'ensemble des $\mathrm{D} \in \Delta\left(\operatorname{supp}\left(\Sigma^{\prime}\right)\right)$ tels que $\rho(\mathrm{D})$ est nul sur $\Sigma \backslash \Sigma^{\prime}$. On dira que la composante $\Sigma^{\prime}$ est effaçable, si :

(1) $\Delta\left(\Sigma^{\prime}\right)$ est un sous-ensemble distingué lisse de $\Delta$; 
(2) $\Sigma\left(\Delta\left(\Sigma^{\prime}\right)\right)=\Sigma^{\prime}$

(On rappelle que, pour tout sous-ensemble distingué $\Delta^{\prime}$ de $\Delta$, on a posé $\Sigma\left(\Delta^{\prime}\right)=\Sigma \backslash\left(\Sigma / \Delta^{\prime}\right)$, voir 3.5.)

Une composante $\Delta$-connexe $\Sigma^{\prime}$ de $\Sigma$ sera dite isolée si $\operatorname{supp}(\Sigma)=\operatorname{supp}\left(\Sigma^{\prime}\right) \cup$ $\operatorname{supp}\left(\Sigma \backslash \Sigma^{\prime}\right)$ est une factorisation du système (cuspidal) qu'on obtient en localisant $\mathrm{S}^{p}, \Sigma$, $\mathbf{A}$ en $\operatorname{supp}(\Sigma)$. Toute composante isolée est effaçable.

Proposition 4.4 - Soit $\mathrm{S}^{p}, \Sigma, \mathbf{A}$ un système sphérique (adjoint de type A) sans couleur projective. Soit $\Sigma^{\prime}$ une composante $\Delta$-connexe de $\Sigma$.

1) Si le localisé de $\mathrm{S}^{p}, \Sigma, \mathbf{A}$ en supp $\Sigma^{\prime}$ est isomorphe à ao(n), $a c(n), a a(p+q+p), a a(p, p)$, $a a^{*}(p+1+p), a x(1+p+1+q+1), a x(1+p+1,1), a x(1,1,1), a y^{\sim}(p+q+p), a e_{6}(4)$ et $a e_{7}(5)$, alors $\Sigma^{\prime}$ est isolée.

2) Si le localisé de $\mathrm{S}^{p}, \Sigma, \mathbf{A}$ en supp $\Sigma^{\prime}$ n'est pas isomorphe à aa(p) ou à $a c^{*}(n)$ (n pair), alors $\Sigma^{\prime}$ est effaçable.

Preuve - La vérification de (1) se fait sans trop de peine, cas par cas, grâce à la table des systèmes de rang 2. D’après la proposition 4.3, pour montrer (2), il reste à considérer les cas où le localisé en supp $\Sigma^{\prime}$ est isomorphe à $a c^{*}(n)$ ( $n$ impair), $a x(1+p+1), a y(p+q+p), a y(p+q+p-1)$, ay $(p, p), a y(p, p-1), a y^{*}(2+q+2), a z(3,3)$, $a z(3,2), a z(3,1), a e_{6}(6), a e_{6}(5), a e_{7}(7), a e_{7}(6)$ et $a f(4)$.

On vérifie alors également cas par cas, sans trop de peine, que $\Sigma^{\prime}$ est effaçable.

Remarque - Mêmes hypothèses que dans la proposition 4.4. Si $\Sigma \neq \varnothing$, il existe toujours au moins une composante $\Delta$-connexe effaçable. En effet, on peut supposer que le système est cuspidal, et que les sous-systèmes engendrés par les composantes $\Delta$-connexes sont tous isomorphes à $a a(p)$ ou $a c^{*}(n)$ ( $n$ pair). Alors celles dont le support contient une racine simple située sur un des « bouts » du diagramme de Dynkin sont effaçables.

\section{\$5 Preuve du théorème 1}

\subsection{L'application du théorème 1 est bien définie}

Soit $\mathrm{G}$ un groupe semi-simple adjoint quelconque, et soit $\mathrm{X}$ une G-variété magnifique.

Comment s'assurer que le triplet $S_{\mathrm{X}}^{p}, \Sigma_{\mathrm{X}}, \mathbf{A}_{\mathrm{X}}$ possède les propriétés des systèmes sphériques? Vu la définition de ces systèmes, il suffit de vérifier que, pour tout $\Sigma^{\prime} \subset \Sigma_{\mathrm{X}}$ contenant deux éléments, le sous-triplet de $\mathrm{S}_{\mathrm{X}}^{p}, \Sigma_{\mathrm{X}}, \mathbf{A}_{\mathrm{X}}$ obtenu par localisation en $\Sigma^{\prime}$ (voir 3.1) possède les propriétés des systèmes sphériques. Mais ces localisés sont encore 
les triplets de variétés magnifiques (loc. cit.). Les variétés magnifiques de rang 2 étant connues ([Wa]), la vérification se fait cas par cas.

Pour certaines des propriétés des systèmes sphériques, il y a aussi des arguments plus directs : par exemple, la propriété (A2) peut se déduire (suivant une idée de M. Brion) de la dualité entre courbes et diviseurs dans X (voir [Lu2]).

\subsection{Les cas primitifs classiques}

On se place maintenant dans le cadre adjoint de type A. Dans ce numéro et les deux suivants, on va montrer que tout système sphérique primitif est le système sphérique d'une variété magnifique unique (à isomorphisme près).

Autrement dit, si $\mathrm{G}$ est un groupe semi-simple associé à $\mathrm{S}$, on va voir que, pour tout système sphérique primitif $\mathrm{S}^{p}, \Sigma, \mathbf{A}$, il existe un sous-groupe magnifique $\mathrm{H}$ de $\mathrm{G}$ (contenant le centre $\mathrm{G}(\mathrm{G})$ de $\mathrm{G}$ ), unique à conjugaison près, tel que $\mathrm{S}^{p}, \Sigma$, A soit le système sphérique (du plongement magnifique) de $\mathrm{G} / \mathrm{H}$. On appellera $\mathrm{G} / \mathrm{H}$ aussi une « réalisation géométrique » de $\mathrm{S}^{p}, \Sigma, \mathbf{A}$.

Le cas des systèmes classiques est essentiellement connu. En effet, le travail [Vu2] peut s'interpréter comme le calcul des systèmes sphériques des espaces homogènes symétriques. En ne regardant que la partie de [Vu2] qui concerne les groupes de type $\mathrm{A}$, on voit que les systèmes primitifs classiques sont " réalisés géométriquement » par les $\mathrm{G} / \mathrm{H}$ suivants :

(1) $a o(n), n \geqslant 1$

$$
\mathrm{H}=\mathrm{N}_{\mathrm{G}}\left(\mathrm{SO}_{n+1}(\mathbf{C})\right) \text { et } \mathrm{G}=\mathrm{SL}_{n+1}(\mathbf{C})
$$

(2) $a c(n), n$ impair $\geqslant 3$

$$
\mathrm{H}=\mathrm{N}_{\mathrm{G}}\left(\mathrm{S} p_{n+1}(\mathbf{G})\right) \text { et } \mathrm{G}=\mathrm{SL}_{n+1}(\mathbf{C})
$$

(3) $a a(p+q+p), n=2 p+q, p \geqslant 1, q \geqslant 1$

$$
\mathrm{H}=\mathrm{N}_{\mathrm{G}}\left(\mathrm{SL}_{p+q}(\mathbf{C}) \times \mathrm{SL}_{p+1}(\mathbf{C})\right)^{0} \cdot \mathrm{C}(\mathrm{G}) \text { et } \mathrm{G}=\mathrm{SL}_{n+1}(\mathbf{G})
$$

(4) $a a(p, p), p \geqslant 1$

$$
\begin{aligned}
& \mathrm{H}=\left(\text { la diagonale de } \mathrm{SL}_{p+1}(\mathbf{G}) \times \mathrm{SL}_{p+1}(\mathbf{G})\right) \cdot \mathrm{C}(\mathrm{G}) \text { et } \\
& \mathrm{G}=\mathrm{SL}_{p+1}(\mathbf{G}) \times \mathrm{SL}_{p+1}(\mathbf{G})
\end{aligned}
$$

(5) $a a(q), q \geqslant 1$

$$
\mathrm{H}=\mathrm{GL}_{q}(\mathbf{G}) \text { et } \mathrm{G}=\mathrm{SL}_{q+1}(\mathbf{G})
$$

(6) $a a^{*}(p+1+p), n=2 p+1, p \geqslant 1$

$$
\mathrm{H}=\mathrm{N}_{\mathrm{G}}\left(\mathrm{SL}_{p+1}(\mathbf{G}) \times \mathrm{SL}_{p+1}(\mathbf{G})\right) \text { et } \mathrm{G}=\mathrm{SL}_{n+1}(\mathbf{C})
$$


Ge qui précède montre l'existence d'une réalisation géométrique pour les systèmes sphériques classiques. Pour l'unicité, on peut raisonner comme suit. Si G/H est une réalisation d'un système sphérique classique, alors $\mathrm{H}$ est réductif. En effet, cela résulte du fait que tout système classique $\mathrm{S}^{p}, \Sigma, \mathbf{A}$ vérifie :

(1) $0 \notin \rho(\Delta)$; et

(2) il existe une forme linéaire sur $\mathrm{N}$ qui est $>0$ sur $\rho(\Delta)$ et $\leqslant 0$ sur $\mathrm{V}$

(où $\rho: \Delta \rightarrow \mathrm{N} \supset \mathrm{V}$ est l'espace vectoriel colorié de $\mathrm{S}^{p}, \Sigma, \mathbf{A}$ qui s'identifie à l'espace vectoriel colorié de $\mathrm{G} / \mathrm{H}$ ), conditions qui sont équivalentes à la réductivité de $\mathrm{H}$ (voir ([Br6]). Or les sous-groupes sphériques réductifs sont connus ([Mi], [Br2]). Seuls les sous-groupes réductifs magnifiques (d'un groupe adjoint $\mathrm{G}$ de type $\mathrm{A}$ ) nous concernent ici. Pour chacun des groupes $G$ des six systèmes (1) à (6), il n'y a qu'un nombre fini de tels sous-groupes. On constate que, dans chaque cas, le $\mathrm{H}$ ci-dessus est le seul (à conjugaison près) parmi ces sous-groupes, vérifiant les formules (vraies pour tout sous-groupe sphérique) :

$$
\begin{aligned}
& \operatorname{dim} \mathrm{G} / \mathrm{H}=\operatorname{dim} \mathrm{G}_{-\mathrm{S}^{p}}^{u}+\operatorname{card}(\Sigma) \text { et } \\
& \operatorname{rang} \Xi(\mathrm{H})=\operatorname{card}(\Delta)-\operatorname{card}(\Sigma) .
\end{aligned}
$$

\subsection{Les cas primitifs non classiques : "l'unicité "}

Montrons maintenant l'unicité, à isomorphisme près, des réalisations géométriques pour les systèmes sphériques primitifs non classiques.

Nous allons procéder par vérification cas par cas. Voici l'argument type qu'on utilisera (dans presque tous les cas) pour prouver l'unicité des réalisations géométriques. Soit $\mathrm{S}^{p}, \Sigma, \mathbf{A}$ un système sphérique primitif non classique, et soit $\mathrm{G} / \mathrm{H}$ une réalisation géométrique de $\mathrm{S}^{p}, \Sigma, \mathbf{A}$. On donnera, dans chaque cas, explicitement un sous-ensemble $\Delta^{\prime} \subset \Delta$ distingué vérifiant : classique;

a) $\left(\mathrm{S}^{p}, \Sigma, \mathbf{A}\right) / \Delta^{\prime}$ est ou bien parabolique, ou bien l'induit parabolique d'un système

b) il existe un sous-ensemble $\Delta^{\prime \prime}$ distingué parabolique minimal de $\Delta$ contenant $\Delta^{\prime}$.

D'après 5.2 , au système $\left(\mathrm{S}^{p}, \Sigma, \mathbf{A}\right) / \Delta^{\prime}$ correspond un sous-groupe magnifique $\mathrm{H}_{1}$ unique (à conjugaison près). On peut supposer $\mathrm{H} \subset \mathrm{H}_{1}$. On constatera que $\mathrm{H}_{1}$ est toujours connexe. Choisissons des décompositions de Levi $\mathrm{H}=\mathrm{LH}^{u}$ et $\mathrm{H}_{1}=\mathrm{L}_{1} \mathrm{H}_{1}^{u}$ telles que $\mathrm{L} \subset \mathrm{L}_{1}$, et notons $\mathrm{C}$ et $\mathrm{C}_{1}$ les centres connexes de $\mathrm{L}$ et $\mathrm{L}_{1}$. D'après a) $\mathrm{H}_{1}^{u}$ est le radical unipotent du groupe parabolique $\mathrm{Q}=\mathrm{N}_{\mathrm{G}}\left(\mathrm{H}_{1}^{u}\right)$. De b) résulte que $(\mathrm{L}, \mathrm{L})=\left(\mathrm{L}_{1}, \mathrm{~L}_{1}\right)$, d'où il suit que $\mathrm{H}^{u} \subset \mathrm{H}_{1}^{u}$. Alors $\operatorname{dim} \mathrm{C}=\operatorname{rang} \Xi(\mathrm{H})=\operatorname{card}(\Delta)-\operatorname{card}(\Sigma)$. Dans chaque cas, on calculera $\operatorname{dimH}_{1}^{u} / \mathrm{H}^{u}$ qui se révélera " petit » (par exemple, si $\mathrm{S}^{p}=\mathrm{S}^{p} / \Delta_{1}$, ce qui arrivera souvent, alors $\left.\operatorname{dim} \mathrm{H}_{1}^{u} / \mathrm{H}^{u}=\operatorname{card}\left(\Delta_{1}\right)\right)$. Comme $\mathrm{LieH}_{1}^{u}$ est un 
$\mathrm{L}_{1}$-module sans multiplicité, ce qui précède ne laissera alors qu'une possibilité pour $\mathrm{C}$ et $\mathrm{H}_{1}^{u}$ (à conjugaison près).

Passons donc en revue, cas par cas, les systèmes primitifs non classiques.

(7) $a c^{*}(n), n \geqslant 3$

Posons $\Delta^{\prime}=\left\{\mathrm{D}_{\alpha_{i}}, i\right.$ pair, $\left.1 \leqslant 1 \leqslant n\right\}$; c'est un sous-ensemble distingué de $\Delta$.

1) Si $n$ est pair, le triplet quotient $\left(S^{p}, \Sigma, \mathbf{A}\right) / \Delta^{\prime}$ est donné par :

$$
\begin{aligned}
& \mathrm{S}^{p} / \Delta^{\prime}=\left\{\alpha_{i}, i \text { pair, } 1 \leqslant i \leqslant n\right\} \\
& \Sigma / \Delta^{\prime}=\left\{\alpha_{2}+2 \alpha_{3}+\alpha_{4}, \ldots, \alpha_{n-2}+2 \alpha_{n-1}+\alpha_{n}\right\} \\
& \mathbf{A} / \Delta^{\prime}=\varnothing .
\end{aligned}
$$

On a $\operatorname{dim} \mathrm{C}=\operatorname{dim} \mathrm{C}_{1}=1$ et $\operatorname{dim} \mathrm{H}_{1}^{u} / \mathrm{H}^{u}=n=\operatorname{dim} \mathrm{H}_{1}^{u}$, d'où $\mathrm{H}=\mathrm{L}=\mathrm{L}_{1}$. Par suite, si $\mathrm{G}=\mathrm{SL}_{n+1}(\mathbf{G})$, alors $\mathrm{H}=\mathrm{N}_{\mathrm{G}}\left(\mathrm{S}_{n}(\mathbf{G})\right)$ (où $\mathrm{S}_{n}(\mathbf{G}) \subset \mathrm{SL}_{n}(\mathbf{G}) \subset \mathrm{SL}_{n+1}(\mathbf{C})$ sont les inclusions naturelles).

2) Si $n$ est impair, le triplet quotient $\left(\mathrm{S}^{p}, \Sigma, \mathbf{A}\right) / \Delta^{\prime}$ est donné par :

$$
\begin{aligned}
& \mathrm{S}^{p} / \Delta^{\prime}=\left\{\alpha_{i}, i \text { pair }, 1 \leqslant i \leqslant n\right\} \\
& \Sigma / \Delta^{\prime}=\left\{\alpha_{2}+2 \alpha_{3}+\alpha_{4}, \ldots, \alpha_{n-3}+2 \alpha_{n-2}+\alpha_{n-1}\right\} \\
& \mathbf{A} / \Delta^{\prime}=\varnothing
\end{aligned}
$$

On a $\operatorname{dim} \mathrm{C}=1, \operatorname{dim} \mathrm{C}_{1}=2$ et $\operatorname{dim}_{1}^{u} / \mathrm{H}^{u}=n-1 . \mathrm{Le}_{1}$-module $\mathrm{LieH}_{1}^{u}$ se décompose en trois modules irréductibles, l'un de dimension 1, et deux de dimension $n-1$, isomorphes en tant que $\left(\mathrm{L}_{1}, \mathrm{~L}_{1}\right)$-module et dans lesquels $\mathrm{C}_{1}$ opère avec les poids $\alpha_{1}$ et $\alpha_{n}$. De ce qui précède, on déduit que $\mathrm{C}=\left\{c \in \mathrm{G}_{1}, \alpha_{1}(c)=\alpha_{n}(c)\right\}$, et que $\mathrm{LieH}^{u}$ est un sous- $\left(\mathrm{L}_{1}\right.$, $\mathrm{L}_{1}$ )-module de codimension $n-1$ dans $\mathrm{LieH}_{1}^{u}$, non stable par $\mathrm{C}_{1}$, ce qui détermine $\mathrm{H}$ à conjugaison près.

(8) $a x(1+p+1+q+1), n=p+q+3, p \geqslant 1, q \geqslant 1$

L'ensemble $\Delta$ est ici composé de 7 éléments : de $\mathrm{D}_{\alpha_{1}}^{+}=\mathrm{D}_{\alpha_{p+2}}^{+}, \mathrm{D}_{\alpha_{1}}^{-}=\mathrm{D}_{\alpha_{n}}^{-}$, $\mathrm{D}_{\alpha_{p+2}}^{-}=\mathrm{D}_{\alpha_{n}}^{+}$et si $p>1$, de $\mathrm{D}_{\alpha_{2}}$ et $\mathrm{D}_{\alpha_{p+1}}$ (et si $p=1$, de $\mathrm{D}_{\alpha_{2}}^{+}$et $\mathrm{D}_{\alpha_{2}}^{-}$) et si $q>1$, de $\mathrm{D}_{\alpha_{p+3}}$ et $\mathrm{D}_{\alpha_{n-1}}$ (et si $q=1$, de $\mathrm{D}_{\alpha_{p+3}}^{+}$et $\mathrm{D}_{\alpha_{p+3}}^{-}$).

Si $p>1$, posons $\Delta^{\prime}=\left\{\mathrm{D}_{\alpha_{1}}^{+}, \mathrm{D}_{\alpha_{1}}^{-}, \mathrm{D}_{\alpha_{2}}\right\}$ (et si $p=1$, posons $\Delta^{\prime}=\left\{\mathrm{D}_{\alpha_{1}}^{+}, \mathrm{D}_{\alpha_{1}}^{-}, \mathrm{D}_{\alpha_{2}}^{+}\right\}$). Alors $\Delta^{\prime}$ est distingué, et le triplet quotient $\left(\mathrm{S}^{p}, \Sigma, \mathbf{A}\right) / \Delta^{\prime}$ est

$$
\begin{aligned}
& \mathrm{S}^{p} / \Delta^{\prime}=\left\{\alpha_{1}, \ldots, \alpha_{p}, \alpha_{p+4}, \ldots, \alpha_{n-2}\right\} \\
& \Sigma / \Delta^{\prime}=\left\{\alpha_{p+2}+\alpha_{n}, \alpha_{p+3}+\ldots+\alpha_{n-1}\right\}
\end{aligned}
$$

$\mathbf{A} / \Delta^{\prime}=\varnothing$ si $q>1$ (et si $q=1$, alors $\mathbf{A} / \Delta^{\prime}$ est concentré en $\alpha_{p+3}$, et sans élément projectif). 
Dans les deux cas, on a $\operatorname{dim} \mathrm{C}=\operatorname{dim} \mathrm{G}_{1}=2$, donc $\mathrm{L}=\mathrm{L}_{1}$. Si $\mathrm{G}=\mathrm{SL}_{n+1}(\mathbf{G})$, on a donc $\mathrm{L}=\mathrm{N}_{\mathrm{G}}\left(\mathrm{SL}_{p+1}(\mathbf{G}) \times \mathrm{SL}_{2}(\mathbf{G}) \times \mathrm{SL}_{q+1}(\mathbf{C})\right)$. Le $\mathrm{L}_{1}$-module $\mathrm{LieH}_{1}^{u}$ se décompose en deux modules irréductibles, l'un de dimension $(p+1)(q+1)$ et l'autre de dimension $2(q+1)$. Puisque $\operatorname{dim} \mathrm{H}_{1}^{u} / \mathrm{H}^{u}=2(q+1)$, il est clair que Lie $\mathrm{H}^{u}$ est égal à celui de dimension $(p+1)(q+1)$, ce qui détermine $\mathrm{H}$ (à conjugaison près : en effet, si $q=1$, les deux choix conduisent à des sous-groupes conjugués).

(9) $a x(1+p+1,1), p \geqslant 1$

L'ensemble $\Delta$ est ici composé de 5 éléments : de $\mathrm{D}_{\alpha_{1}}^{+}=\mathrm{D}_{\alpha_{p+2}}^{+}, \mathrm{D}_{\alpha_{1}}^{-}=\mathrm{D}_{\beta_{1}}^{-}$, $\mathrm{D}_{\alpha_{p+2}}^{-}=\mathrm{D}_{\beta_{1}}^{+}$et si $p>1$, de $\mathrm{D}_{\alpha_{2}}$ et $\mathrm{D}_{\alpha_{p+1}}$ (et si $p=1$, de $\mathrm{D}_{\alpha_{2}}^{+}$et $\mathrm{D}_{\alpha_{2}}^{-}$).

Si $p>1$, posons $\Delta^{\prime}=\left\{\mathrm{D}_{\alpha_{1}}^{+}, \mathrm{D}_{\alpha_{1}}^{-}, \mathrm{D}_{\alpha_{2}}\right\}$ (et si $p=1$, posons $\Delta^{\prime}=\left\{\mathrm{D}_{\alpha_{1}}^{+}, \mathrm{D}_{\alpha_{1}}^{-}, \mathrm{D}_{\alpha_{2}}^{+}\right\}$). Alors $\Delta^{\prime}$ est distingué, et le triplet quotient $\left(\mathrm{S}^{p}, \Sigma, \mathbf{A}\right) / \Delta^{\prime}$ est

$$
\begin{aligned}
& \mathrm{S}^{p} / \Delta^{\prime}=\left\{\alpha_{1}, \ldots, \alpha_{p}\right\} \\
& \Sigma / \Delta^{\prime}=\left\{\alpha_{p+2}+\beta_{1}\right\} \\
& \mathbf{A} / \Delta^{\prime}=\varnothing .
\end{aligned}
$$

On a $\operatorname{dim} \mathrm{C}=\operatorname{dim} \mathrm{C}_{1}=1$ et $\operatorname{dim} \mathrm{H}_{1}^{u} / \mathrm{H}^{u}=2(p+1)=\operatorname{dim} \mathrm{H}_{1}^{u}$, d'où $\mathrm{H}=\mathrm{L}_{=}=\mathrm{L}_{1}$. Si $\mathrm{G}=\mathrm{SL}_{p+3}(\mathbf{G}) \times \mathrm{SL}_{2}(\mathbf{G})$, on a donc $\mathrm{H}=\mathrm{N}_{\mathrm{G}}\left(\mathrm{SL}_{p+1}(\mathbf{C}) \times \mathrm{SL}_{2}(\mathbf{G})\right)$, où l'on se sert du plongement diagonal de $\mathrm{SL}_{2}(\mathbf{G})$ dans $\mathrm{SL}_{2}(\mathbf{G}) \times \mathrm{SL}_{2}(\mathbf{C})$ et de l'inclusion naturelle $\mathrm{SL}_{p+1}(\mathbf{G}) \times \mathrm{SL}_{2}(\mathbf{C}) \subset \mathrm{SL}_{p+3}(\mathbf{G})$, pour plonger $\mathrm{SL}_{p+1}(\mathbf{C}) \times \mathrm{SL}_{2}(\mathbf{G})$ dans $\mathrm{SL}_{p+1}(\mathbf{G}) \times \mathrm{SL}_{2}(\mathbf{G}) \times$ $\mathrm{SL}_{2}(\mathbf{C})$, puis dans $\mathrm{G}$.

(10) $\operatorname{ax}(1+p+1), p \geqslant 1$

L'ensemble $\Delta$ est ici composé de 5 éléments : de $\mathrm{D}_{\alpha_{1}}^{+}=\mathrm{D}_{\alpha_{p+2}}^{+}, \mathrm{D}_{\alpha_{1}}^{-}, \mathrm{D}_{\alpha_{p+2}}^{-}$et si $p>1$, de $\mathrm{D}_{\alpha_{2}}$ et $\mathrm{D}_{\alpha_{p+1}}$ (et si $p=1$, de $\mathrm{D}_{\alpha_{2}}^{+}$et $\mathrm{D}_{\alpha_{2}}^{-}$).

Si $p>1$, posons $\Delta^{\prime}=\left\{\mathrm{D}_{\alpha_{1}}^{+}, \mathrm{D}_{\alpha_{2}}, \mathrm{D}_{\alpha_{p+1}}\right\}$ (et si $p=1$, posons $\Delta^{\prime}=\left\{\mathrm{D}_{\alpha_{1}}^{+}, \mathrm{D}_{\alpha_{2}}^{+}, \mathrm{D}_{\alpha_{2}}^{-}\right\}$). Alors $\Delta^{\prime}$ est distingué, et le triplet quotient $\left(\mathrm{S}^{p}, \Sigma, \mathbf{A}\right) / \Delta^{\prime}$ est

$$
\begin{aligned}
& S^{p} / \Delta^{\prime}=\left\{\alpha_{2}, \ldots, \alpha_{p+2}\right\} \\
& \Sigma / \Delta^{\prime}=\varnothing \\
& \mathbf{A} / \Delta^{\prime}=\varnothing .
\end{aligned}
$$

On a $\operatorname{dim} \mathrm{C}=\operatorname{dim} \mathrm{G}_{1}=2$, donc $\mathrm{L}=\mathrm{L}_{1}$. Si $\mathrm{G}=\mathrm{SL}_{p+3}(\mathbf{G})$, on peut prendre $\mathrm{L}=\mathrm{N}_{\mathrm{G}}\left(\mathrm{SL}_{p+1}(\mathbf{G})\right)$. Le $\mathrm{L}_{1}$-module $\mathrm{LieH}_{1}^{u}$ se décompose en trois modules irréductibles, l'un de dimension 1 et les deux autres de dimension $p+1$. Puisque $\operatorname{dim} \mathrm{H}_{1}^{u} / \mathrm{H}^{u}=2(p+1)$, il suit que $\mathrm{LieH}^{u}$ doit être celui de dimension 1, ce qui détermine $\mathrm{H}$ (à conjugaison près). 
(11) $\operatorname{ax}(1,1,1)$

Le groupe $\mathrm{H}$ est réductif, de dimension 3, et se projette surjectivement sur tout facteur de $\mathrm{G}$. Si $\mathrm{G}=\mathrm{SL}_{2}(\mathbf{C}) \times \mathrm{SL}_{2}(\mathbf{G}) \times \mathrm{SL}_{2}(\mathbf{G})$, cela ne laisse qu'une seule possibilité (à conjugaison près) : $\mathrm{H}=\left(\right.$ la diagonale de $\left.\mathrm{SL}_{2}(\mathbf{G}) \times \mathrm{SL}_{2}(\mathbf{G}) \times \mathrm{SL}_{2}(\mathbf{G})\right) \cdot \mathrm{G}(\mathrm{G})$.

(12) ay $(p+q+p), n=2 p+q, p \geqslant 2, q \geqslant 1$

L'ensemble $\Delta$ est ici composé de $2 p+3$ éléments : de $\mathrm{D}_{\alpha_{1}}^{+}=\mathrm{D}_{\alpha_{n-i+1}}^{+}(1 \leqslant i \leqslant p)$, de $\mathrm{D}_{\alpha_{i}}^{-}=\mathrm{D}_{\alpha_{n-i}}^{-}(1 \leqslant i \leqslant p-1)$, de $\mathrm{D}_{\alpha_{p}}^{-}$et de $\mathrm{D}_{\alpha_{n}}^{-}$; et si $q>1$, de $\mathrm{D}_{\alpha_{p+1}}$ et $\mathrm{D}_{\alpha_{p+q}}$ (ou si $q=1$, de $\mathrm{D}_{\alpha_{p+1}}^{+}$et $\mathrm{D}_{\alpha_{p+1}}^{-}$; pour distinguer les deux, on convient que $\left.<\rho\left(\mathrm{D}_{\alpha_{p+1}}^{-}\right), \alpha_{p}>=-1\right)$.

Posons $\Delta^{\prime}=\left\{\mathrm{D}_{\alpha_{i}}^{-}=\mathrm{D}_{\alpha_{n-i}}^{-}(1 \leqslant i \leqslant p-1), \mathrm{D}_{\alpha_{p}}^{-}, \mathrm{D}_{\alpha_{n}}^{-}\right\}$. Alors $\Delta^{\prime}$ est distingué, et le triplet quotient $\left(\mathrm{S}^{p}, \Sigma, \mathbf{A}\right) / \Delta^{\prime}$ est isomorphe à $a a(p+q+p)$ (on s'écarte ici de l'argument type, car la condition b) n'est pas remplie). $\mathrm{Si} \mathrm{G}=\mathrm{SL}_{n+1}(\mathbf{C})$, on a $\mathrm{H}_{1}=\mathrm{N}_{\mathrm{G}}\left(\mathrm{SL}_{p+1}(\mathbf{C}) \times \mathrm{SL}_{p+q}(\mathbf{G})\right)^{0}$. Un calcul de dimension donne $\operatorname{dimH}_{1} / \mathrm{H}=p$, d'où l'on déduit que $\mathrm{H}=\mathrm{N}_{\mathrm{G}}\left(\mathrm{P} \times \mathrm{SL}_{p+q}(\mathbf{C})\right)$, où $\mathrm{P}$ est un sous-groupe (parabolique) de codimension $p$ de $\mathrm{SL}_{p+1}(\mathbf{C})$. Il y a deux classes de conjugaison de tels sous-groupes dans $\mathrm{SL}_{p+1}(\mathbf{C})$. Comme $\Delta \backslash\left\{\mathrm{D}_{\alpha_{p}}^{-}\right\}$est distingué parabolique dans $\Delta$, le groupe $\mathrm{H}$ est contenu dans un conjugué de $\mathrm{G}_{\mathrm{S} \backslash\left\{\alpha_{p}\right\}}$, ce qui détermine $\mathrm{P}$ (et $\mathrm{H}$ ) à conjugaison près.

(13) $a y(p+q+p-1), p \geqslant 2, q \geqslant 1$

L'ensemble $\Delta$ est ici composé de $2 p+1$ éléments : de $\mathrm{D}_{\alpha_{1}}^{+}$, de $\mathrm{D}_{\alpha_{1}}^{+}=\mathrm{D}_{\alpha_{n-i+1}}^{+}$ $(2 \leqslant i \leqslant p)$, de $\mathrm{D}_{\alpha_{i}}^{-}=\mathrm{D}_{\alpha_{n-i}}^{-}(1 \leqslant i \leqslant p-1)$, et de $\mathrm{D}_{\alpha_{p}}^{-}$; et si $q>1$, de $\mathrm{D}_{\alpha_{p+1}}$ et $\mathrm{D}_{\alpha_{p+q}}$ (ou si $q=1$, de $\mathrm{D}_{\alpha_{p+1}}^{+}$et $\mathrm{D}_{\alpha_{p+1}}^{-}$; pour distinguer les deux, on convient que $\left.<\rho\left(D_{\alpha_{p+1}}^{-}\right), \alpha_{p}>=-1\right)$.

Si $q>1$, posons $\Delta^{\prime}=\Delta \backslash\left\{\mathrm{D}_{\alpha_{1}}^{+}, \mathrm{D}_{\alpha_{p}}^{-}, \mathrm{D}_{\alpha_{p+1}}\right\}$ (et si $q=1$, posons $\Delta^{\prime}=\Delta \backslash\left\{\mathrm{D}_{\alpha_{1}}^{+}\right.$, $\left.\left.\mathrm{D}_{\alpha_{p}}^{-}, \mathrm{D}_{\alpha_{p+1}}^{-}\right\}\right)$. Alors $\Delta^{\prime}$ est distingué, et le triplet quotient $\left(\mathrm{S}^{p}, \Sigma, \mathbf{A}\right) / \Delta^{\prime}$ est

$$
\begin{aligned}
& \mathrm{S}^{p} / \Delta^{\prime}=\left\{\alpha_{2}, \ldots, \alpha_{p-1}, \alpha_{p+2}, \ldots, \alpha_{n}\right\} \\
& \Sigma / \Delta^{\prime}=\left\{\alpha_{1}+\ldots+\alpha_{p}\right\} \\
& \mathbf{A} / \Delta^{\prime}=\varnothing
\end{aligned}
$$

On a $\operatorname{dim} \mathrm{C}=\operatorname{dim} \mathrm{C}_{1}=2$, donc $\mathrm{L}=\mathrm{L}_{1}$. Si $\mathrm{G}=\mathrm{SL}_{n}(\mathbf{G})$, on a donc $\mathrm{L}=\mathrm{N}_{\mathrm{G}}\left(\mathrm{GL}_{p}(\mathbf{G})\right.$ $\left.\times \mathrm{SL}_{p+q-1}(\mathbf{C})\right)$. Le $\mathrm{L}_{1}$-module $\mathrm{LieH}_{1}^{u}$ se décompose en deux modules irréductibles, l'un de dimension $p(p+q-1)$ et l'autre de dimension $p+q-1$. Puisque $\operatorname{dim} \mathrm{H}_{1}^{u} / \mathrm{H}^{u}=p(p+q-1)$, il est clair que $\mathrm{LieH}^{u}$ est égal à celui de dimension $p+q-1$, ce qui détermine $\mathrm{H}$ (à conjugaison près, lorsque $p=1$ ). 
(14) ay $(p, p), p \geqslant 2$

L'ensemble $\Delta$ est ici composé de $2 p+1$ éléments : de $\mathrm{D}_{\alpha_{i}}^{+}=\mathrm{D}_{\beta_{i}}^{+}(1 \leqslant i \leqslant p)$, de $\mathrm{D}_{\alpha_{i}}^{-}=\mathrm{D}_{\beta_{i+1}}^{-}(1 \leqslant i \leqslant p-1)$, et de $\mathrm{D}_{\alpha_{p}}^{-}$et $\mathrm{D}_{\beta_{1}}^{-}$.

Posons $\Delta^{\prime}=\Delta \backslash\left\{\mathrm{D}_{\alpha_{i}}^{-}=\mathrm{D}_{\beta_{i+1}}^{-}(1 \leqslant i \leqslant p-1)\right\}$; cet ensemble est distingué, et le triplet quotient $\left(\mathrm{S}^{p}, \Sigma, \mathbf{A}\right) / \Delta^{\prime}$ est isomorphe à $a a(p, p)$ (on s'écarte ici de l'argument type, car la condition $b)$ n'est pas vérifiée). On sait que $a a(p, p)$ est réalisé géométriquement par $\mathrm{G} / \mathrm{H}_{1}$, où $\mathrm{G}=\mathrm{PGL}_{p+1}(\mathbf{G}) \times \mathrm{PGL}_{p+1}(\mathbf{C})$ et où $\mathrm{H}_{1}$ est la diagonale de $\mathrm{G}$.

Un calcul de dimension donne $\operatorname{dimH}_{1} / \mathrm{H}=p$, ce qui suffit presque pour déterminer H. En effet, $\mathrm{PGL}_{p+1}(\mathbf{C})$ contient exactement deux classes de conjugaison de sous-groupes (paraboliques) $\mathrm{P}$ de codimension $p$. L'ensemble $\Delta \backslash\left\{\mathrm{D}_{\beta_{1}}^{-}\right\}$étant distingué parabolique dans $\Delta$, le groupe $\mathrm{H}$ est contenu dans un conjugué de $\mathrm{G}_{\mathrm{S} \backslash\left\{\beta_{1}\right\}}$, ce qui détermine $\mathrm{P}($ et $\mathrm{H})$ à conjugaison près.

(15) ay $(p, p-1), p \geqslant 2$

L'ensemble $\Delta$ est ici composé de $2 p$ éléments : de $\mathrm{D}_{\alpha_{1}}^{+}$, de $\mathrm{D}_{\alpha_{i}}^{+}=\mathrm{D}_{\beta_{i}}^{+}(2 \leqslant i \leqslant p)$, de $\mathrm{D}_{\alpha_{i}}^{-}=\mathrm{D}_{\beta_{i+1}}^{-}(1 \leqslant i \leqslant p-1)$, et de $\mathrm{D}_{\alpha_{p}}^{-}$.

Posons $\Delta^{\prime}=\Delta \backslash\left\{\mathrm{D}_{\alpha_{i}}^{-}=\mathrm{D}_{\beta_{i+1}}^{-}(1 \leqslant i \leqslant p-1)\right\}$; cet ensemble est distingué, et le triplet quotient $\left(\mathrm{S}^{p}, \Sigma, \mathbf{A}\right) / \Delta^{\prime}$ est

$$
\begin{aligned}
& \mathrm{S}^{p} / \Delta^{\prime}=\varnothing \\
& \Sigma / \Delta^{\prime}=\left\{\alpha_{1}+\beta_{1}, \ldots, \alpha_{p-1}+\beta_{p-1}\right\} \\
& \mathbf{A} / \Delta^{\prime}=\varnothing .
\end{aligned}
$$

On a $\operatorname{dim} \mathrm{C}=\operatorname{dim} \mathrm{C}_{1}=1$ et $\operatorname{dimH}_{1}^{u} / \mathrm{H}^{u}=p=\operatorname{dim} \mathrm{H}_{1}^{u}$, d'où $\mathrm{H}=\mathrm{L}=\mathrm{L}_{1}$. Si $\mathrm{G}=\mathrm{SL}_{p+1}(\mathbf{G}) \times \mathrm{SL}_{p}(\mathbf{C})$, on a donc $\mathrm{H}=\mathrm{N}_{\mathrm{G}}\left(\mathrm{H}^{\prime}\right)$, où $\mathrm{H}^{\prime}$ est la diagonale de $\mathrm{SL}_{p}(\mathbf{G}) \times \mathrm{SL}_{p}(\mathbf{G})$, groupe qu'on plonge dans $\mathrm{G}$ au moyen de l'inclusion naturelle de $\mathrm{SL}_{p}(\mathbf{C})$ dans $\mathrm{SL}_{p+1}(\mathbf{G})$.

(16) La famille $a \tilde{y}^{\sim}(p+q+p), n=2 p+q, p \geqslant 2, q \geqslant 1$

L'ensemble $\Delta$ est ici composé de $2 p+3$ éléments : de $\mathrm{D}_{\alpha_{i}}^{+}=\mathrm{D}_{\alpha_{n-p+i}^{+}}^{+}(1 \leqslant i \leqslant p)$, de $\mathrm{D}_{\alpha_{i}}^{-}=\mathrm{D}_{\alpha_{n-p+i+1}}^{-}(1 \leqslant i \leqslant p-1)$, de $\mathrm{D}_{\alpha_{p}}^{-}$et de $\mathrm{D}_{n-p+1}^{-}$; et si $q>1$, de $\mathrm{D}_{\alpha_{p+1}}$ et $\mathrm{D}_{\alpha_{p+q}}$ (ou si $q=1$, de $\mathrm{D}_{\alpha_{p+1}}^{+}$et $\mathrm{D}_{\alpha_{p+1}}^{-}$; pour distinguer les deux, on convient que $\left.<\rho\left(\mathrm{D}_{\alpha_{p+1}}^{-}\right), \alpha_{p}>=-1\right)$.

Posons $\Delta^{\prime}=\left\{\mathrm{D}_{\alpha_{i}}^{+}, 1 \leqslant i \leqslant p\right\}$. L'ensemble $\Delta^{\prime}$ est distingué et le triplet quotient $\left(\mathrm{S}^{p}, \Sigma, \mathbf{A}\right) / \Delta^{\prime}$ est :

$$
\begin{aligned}
& \mathrm{S}^{p} / \Delta^{\prime}=\left\{\alpha_{p+2}, \ldots, \alpha_{p+q-1}\right\} \\
& \Sigma / \Delta^{\prime}=\left\{\alpha_{1}+\alpha_{n-p+2}, \ldots, \alpha_{p-1}+\alpha_{n}\right\}
\end{aligned}
$$

$\mathbf{A} / \Delta^{\prime}=\varnothing$ si $q>1$ (si $q=1, \mathbf{A} / \Delta^{\prime}$ est composé de deux éléments non projectifs, concentrés en $\left.\alpha_{p+1}\right)$. 
On a $\operatorname{dim} \mathrm{C}=2, \operatorname{dim} \mathrm{C}_{1}=3$ et $\operatorname{dim} \mathrm{H}_{1}^{u} / \mathrm{H}^{u}=p\left(=\operatorname{card} \Delta^{\prime}\right)$. Le $\mathrm{L}_{1}$-module $\mathrm{LieH}_{1}^{u}$ se décompose en six sous-modules irréductibles, de dimensions $1, p^{2}-1, p q, p q, p$ et $p$.

Examinons d'abord le cas où $q>1$. Alors les deux sous-modules de dimension $p$ sont isomorphes en tant que $\left(\mathrm{L}_{1}, \mathrm{~L}_{1}\right)$-module et $\mathrm{G}_{1}$ opère dans ces deux sous-modules au moyen des poids $\delta_{1}=\alpha_{p}$ et $\delta_{2}=\alpha_{p+1}+\ldots+\alpha_{p+q+1}$. De ce qui précède, on déduit que $\mathrm{C}=\left\{c \in \mathrm{C}_{1}, \delta_{1}(c)=\delta_{2}(c)\right\}$, et que $\mathrm{LieH}^{u}$ est un sous- $\left(\mathrm{L}_{1}, \mathrm{~L}_{1}\right)$-module de codimension $p$ dans $\mathrm{LieH}_{1}^{u}$, non stable par $\mathrm{G}_{1}$, ce qui détermine $\mathrm{H}$ à conjugaison près.

Lorsque $q=1$, il y a quatre sous- $\mathrm{L}_{1}$-modules de $\mathrm{LieH}_{1}^{u}$ de dimension $p, \mathrm{~W}_{i}$ $(i=1,2,3,4)$, qui sont isomorphes en tant que $\left(\mathrm{L}_{1}, \mathrm{~L}_{1}\right)$-module. Le groupe $\mathrm{C}_{1}$ opère dans ces sous-modules au moyen des poids $\delta_{1}=\alpha_{p}, \delta_{2}=\alpha_{p+1}+\alpha_{p+2}, \delta_{3}=\alpha_{p}+\alpha_{p+1}$, et $\delta_{4}=\alpha_{p+2}$. Pour tout couple $(i, j)(1 \leqslant i<j \leqslant 4)$ notons $\mathrm{H}_{i j}=\left(\mathrm{L}_{1}, \mathrm{~L}_{1}\right) \mathrm{C}_{i j} \mathrm{H}_{i j}^{u}$ le sousgroupe de $\mathrm{G}$, avec $\mathrm{C}_{i j}=\left\{c \in \mathrm{C}_{1}, \delta_{i}(c)=\delta_{j}(c)\right\}$ et où $\mathrm{LieH}_{i j}^{u}$ est un sous- $\left(\mathrm{L}_{1}, \mathrm{~L}_{1}\right)$-module de codimension $p$ dans $\mathrm{LieH}_{1}^{u}$, non stable par $\mathrm{C}_{1}$, et contenant $\mathrm{W}_{k}$ pour $k \neq i, j$. Le groupe $\mathrm{H}$ est conjugué à l'un des $\mathrm{H}_{i j}$. Les groupes $\mathrm{H}_{13}$ et $\mathrm{H}_{24}$ sont exclus, car ils contiennent le radical d'un sous-groupe parabolique. Le groupe $\mathrm{H}_{14}$ est exclu, car la variété magnifique associée domine une variété magnifique dont le système sphérique s'obtient par induction parabolique à partir de $a y(p, p)$, ce qui n'est pas possible pour $a y^{\sim}(p+1+p)$ (le système sphérique de $\mathrm{G} / \mathrm{H}_{14}$ ne diffère de $a y^{\sim}(p+1+p)$ que par le fait que $\Delta\left(\alpha_{p+1}\right)$ contient un élément projectif), et $\mathrm{H}_{23}$ est conjugué à $\mathrm{H}_{14}$. Par suite $\mathrm{H}$ est égal à $\mathrm{H}_{12}$, ou à $\mathrm{H}_{34}$, qui sont conjugués.

(17) La famille $a y^{*}(2+q+2), n=4+q, q \geqslant 1$

L'ensemble $\Delta$ est ici composé de 7 éléments : de $\mathrm{D}_{\alpha_{1}}^{+}=\mathrm{D}_{\alpha_{n-1}}^{+}$, de $\mathrm{D}_{\alpha_{2}}^{+}=\mathrm{D}_{\alpha_{n}}^{+}$, de $\mathrm{D}_{\alpha_{2}}^{-}=\mathrm{D}_{\alpha_{n-1}}^{-}$, de $\mathrm{D}_{\alpha_{1}}^{-}$et de $\mathrm{D}_{\alpha_{n}}^{-}$; et si $q>1$, de $\mathrm{D}_{\alpha_{3}}$ et $\mathrm{D}_{\alpha_{2+q}}$ (ou si $q=1$, de $\mathrm{D}_{\alpha_{3}}^{+}$et $\mathrm{D}_{\alpha_{3}}^{-}$; pour distinguer les deux, on convient que $\left.<\rho\left(\mathrm{D}_{\alpha_{3}}^{-}\right), \alpha_{2}>=-1\right)$.

Posons $\Delta^{\prime}=\left\{\mathrm{D}_{\alpha_{1}}^{+}, \mathrm{D}_{\alpha_{2}}^{+}\right\}$. L'ensemble $\Delta^{\prime}$ est distingué et le triplet quotient $\left(\mathrm{S}^{p}, \Sigma, \mathbf{A}\right) / \Delta^{\prime}$ est :

$$
\begin{aligned}
& \mathrm{S}^{p} / \Delta^{\prime}=\left\{\alpha_{4}, \ldots, \alpha_{q+1}\right\} \\
& \Sigma / \Delta^{\prime}=\left\{\alpha_{2}+\alpha_{n-1}, \alpha_{3}+\ldots+\alpha_{2+q}\right\} \\
& \mathbf{A} / \Delta^{\prime}=\varnothing \text { si } q>1 \text { (si } q=1, \mathbf{A} / \Delta^{\prime} \text { est composé de deux éléments non } \\
& \text { projectifs, concentrés en } \left.\alpha_{3}\right) .
\end{aligned}
$$

On a $\operatorname{dim} \mathrm{C}=2, \operatorname{dim} \mathrm{C}_{1}=3$ et $\operatorname{dim} \mathrm{H}_{1}^{u} / \mathrm{H}^{u}=2\left(=\operatorname{card} \Delta^{\prime}\right)$. Le $\mathrm{L}_{1}$-module $\mathrm{LieH}_{1}^{u}$ se décompose en quatre sous-modules irréductibles de dimensions $1,2,2, q+1, q+1$.

Examinons d'abord le cas où $q>1$. Alors les deux sous-modules de dimension 2 sont isomorphes en tant que $\left(\mathrm{L}_{1}, \mathrm{~L}_{1}\right)$-module et $\mathrm{G}_{1}$ opère dans ces deux sous-modules au moyen des poids $\delta_{1}=\alpha_{1}$ et $\delta_{2}=\alpha_{3}+\ldots+\alpha_{n}$. De ce qui précède, on déduit que $\mathrm{C}=\left\{c \in \mathrm{C}_{1}, \boldsymbol{\delta}_{1}(c)=\boldsymbol{\delta}_{2}(c)\right\}$, et que Lie $\mathrm{H}^{u}$ est un sous- $\left(\mathrm{L}_{1}, \mathrm{~L}_{1}\right)$-module de codimension 2 dans $\mathrm{LieH}_{1}^{u}$, non stable par $\mathrm{G}_{1}$, ce qui détermine $\mathrm{H}$ à conjugaison près. 
Lorsque $q=1$, il y a quatre sous- $\mathrm{L}_{1}$-modules de $\mathrm{LieH}_{1}^{u}$ de dimension $2, \mathrm{~W}_{i}$ $(i=1,2,3,4)$, qui sont isomorphes en tant que $\left(\mathrm{L}_{1}, \mathrm{~L}_{1}\right)$-module. Le groupe $\mathrm{C}_{1}$ opère dans ces sous-modules au moyen des poids $\delta_{1}=\alpha_{1}, \delta_{2}=\alpha_{3}+\alpha_{4}+\alpha_{5}, \delta_{3}=\alpha_{1}+\alpha_{2}+\alpha_{3}$, et $\delta_{4}=\alpha_{5}$. Pour tout couple $(i, j)(1 \leqslant i<j \leqslant 4)$ notons $\mathrm{H}_{i j}=\left(\mathrm{L}_{1}, \mathrm{~L}_{1}\right) \mathrm{C}_{i j} \mathrm{H}_{i j}^{u}$ le sousgroupe de $\mathrm{G}$, avec $\mathrm{C}_{i j}=\left\{c \in \mathrm{C}_{1}, \delta_{i}(c)=\delta_{j}(c)\right\}$ et où $\operatorname{LieH}_{i j}^{u}$ est un sous- $\left(\mathrm{L}_{1}, \mathrm{~L}_{1}\right)$-module de codimension 2 dans $\mathrm{LieH}_{1}^{u}$, non stable par $\mathrm{C}_{1}$, et contenant $\mathrm{W}_{k}$ pour $k \neq i, j$. Le groupe $\mathrm{H}$ est conjugué à l'un des $\mathrm{H}_{i j}$. Les groupes $\mathrm{H}_{13}$ et $\mathrm{H}_{24}$ sont exclus, car ils contiennent le radical d'un sous-groupe parabolique. Le groupe $\mathrm{H}_{14}$ est exclu, car il est contenu dans un sous-groupe magnifique $\mathrm{H}^{\prime}$, dont l'espace homogène $\mathrm{G} / \mathrm{H}^{\prime}$ a un système sphérique qui s'obtient par induction parabolique à partir de $a y(2,2)$, ce qui n'est pas possible pour $a y^{*}(2+1+2)$ (en fait, $\mathrm{H}_{14}$ est magnifique, mais le système sphérique de $\mathrm{G} / \mathrm{H}_{14}$ diffère de $a y^{*}(2+1+2)$ en $\Delta\left(\alpha_{p+1}\right)$, qui contient un élément projectif), et $\mathrm{H}_{23}$ est conjugué à $\mathrm{H}_{14}$. Par suite, $\mathrm{H}$ est égal à $\mathrm{H}_{12}$ ou à $\mathrm{H}_{34}$, qui sont conjugués.

(18) $a z^{\sim}(3+q+3), q \geqslant 1$

L'ensemble $\Delta$ est ici composé de 9 éléments : de $\mathrm{D}_{\alpha_{1}}^{+}=\mathrm{D}_{\alpha_{3}}^{+}=\mathrm{D}_{\alpha_{q+5}}^{+}$, de $\mathrm{D}_{\alpha_{2}}^{+}=\mathrm{D}_{\alpha_{q+4}}^{+}=\mathrm{D}_{\alpha_{q+6}}^{+}$, de $\mathrm{D}_{\alpha_{2}}^{-}=\mathrm{D}_{\alpha_{q+5}}^{-}$, de $\mathrm{D}_{\alpha_{1}}^{-}, \mathrm{D}_{\alpha_{3}}^{-}, \mathrm{D}_{\alpha_{q+4}}^{-}$et de $\mathrm{D}_{\alpha_{q+6}}^{-}$; et si $q>1$, de $\mathrm{D}_{\alpha_{4}}$ et $\mathrm{D}_{\alpha_{q+3}}$ (ou si $q=1$, de $\mathrm{D}_{\alpha_{4}}^{+}$et $\mathrm{D}_{\alpha_{4}}^{-}$; pour distinguer les deux, on convient que $\left.<\rho\left(\mathrm{D}_{\alpha_{4}}^{-}\right), \alpha_{3}>=-1\right)$.

Posons $\Delta^{\prime}=\left\{\mathrm{D}_{\alpha_{1}}^{+}, \mathrm{D}_{\alpha_{2}}^{+}\right\}$. L'ensemble $\Delta^{\prime}$ est distingué et le triplet quotient $\left(\mathrm{S}^{p}, \Sigma, \mathbf{A}\right) / \Delta^{\prime}$ est :

$$
\mathrm{S}^{p} / \Delta^{\prime}=\left\{\alpha_{5}, \ldots, \alpha_{q+2}\right\}
$$

$\Sigma / \Delta^{\prime}=\left\{\alpha_{2}+\alpha_{q+5}, \alpha_{4}+\ldots+\alpha_{q+3}\right\}$

$\mathbf{A} / \Delta^{\prime}=\varnothing$ si $q>1$ (si $q=1, \mathbf{A} / \Delta^{\prime}$ est concentré en $\alpha_{4}$, et sans élément projectif).

On a $\operatorname{dim} \mathrm{C}=2, \operatorname{dim} \mathrm{C}_{1}=5$ et $\operatorname{dim}_{1}^{u} / \mathrm{H}^{u}=2\left(=\operatorname{card} \Delta^{\prime}\right)$. Le $\mathrm{L}_{1}$-module $\mathrm{LieH}_{1}^{u}$ se décompose en 14 sous-modules irréductibles $\mathrm{W}_{i}(i=1, \ldots, 14)$. Rangeons ces sousmodules de façon à ce que les six premiers engendrent $\mathrm{LieH}_{1}^{u}$ comme algèbre de Lie, et que leurs poids sous $\mathrm{G}_{1}$ soient donnés par : $\delta_{1}=\alpha_{1}, \delta_{2}=\alpha_{3}, \delta_{3}=\alpha_{3}+\alpha_{4}, \delta_{4}=\alpha_{4}+\alpha_{q+4}$, $\delta_{5}=\alpha_{q+4}$ et $\delta_{6}=\alpha_{q+6}$. Alors les $\mathrm{W}_{i}(i=1,2,4,6)$ sont de dimension 2 et les deux autres de dimension $2 q$.

Examinons d'abord le cas où $q>1$. Alors les $\mathrm{W}_{i}(i=1,2,4,6)$ sont isomorphes en tant que $\left(\mathrm{L}_{1}, \mathrm{~L}_{1}\right)$-module. De ce qui précède, on déduit que $\mathrm{C}=\left\{c \in \mathrm{C}_{1}\right.$, $\left.\delta_{1}(c)=\delta_{2}(c)=\delta_{4}(c)=\delta_{6}(c)\right\}$, et que $\mathrm{LieH}^{u}$ est un sous- $\left(\mathrm{L}_{1}, \mathrm{~L}_{1}\right)$-module de codimension 2 dans $\mathrm{LieH}_{1}^{u}$, contenant les $\mathrm{W}_{i}(i \neq 1,2,4,6)$, mais ne contenant aucun des $\mathrm{W}_{i}(i=1,2,4,6)$, ce qui détermine $\mathrm{H}$ à conjugaison près.

Lorsque $q=1$, les $\mathrm{W}_{i}(i=1, \ldots, 6)$ sont isomorphes en tant que $\left(\mathrm{L}_{1}, \mathrm{~L}_{1}\right)$-modules. Pour tout couple $(i, j)(1 \leqslant i<j \leqslant 6)$ notons $\mathrm{H}_{i j}=\left(\mathrm{L}_{1}, \mathrm{~L}_{1}\right) \mathrm{C}_{i j} \mathrm{H}_{i j}^{u}$ le sous-groupe de $\mathrm{G}$, 
où $\mathrm{C}_{i j}$ est défini par $\left\{c \in \mathrm{C}_{1}, \delta_{r}(c)=\delta_{s}(c)\right.$, pour $\left.r, s \in\{1, \ldots, 6\} \backslash\{i, j\}\right\}$, et où Lie $\mathrm{H}_{i j}^{u}$ est un sous- $\left(\mathrm{L}_{1}, \mathrm{~L}_{1}\right)$-module de codimension 2 dans Lie $\mathrm{H}_{1}^{u}$, contenant les $\mathrm{W}_{k}(k>6)$, $\mathrm{W}_{i}$ et $\mathrm{W}_{j}$, mais ne contenant aucun des autres $\mathrm{W}_{k}$. Le groupe $\mathrm{H}$ est conjugué à l'un des $\mathrm{H}_{i j}$. Si $i=1$, ou si $j=6$, ou si $(i, j)=(2,3)$ ou $(4,5)$, alors $\mathrm{H}_{i j}$ contient le radical d'un parabolique, ce qui est exclu. Le groupe $\mathrm{H}_{25}$ est exclu, car il est contenu dans un sous-groupe magnifique $\mathrm{H}^{\prime}$, dont l'espace homogène $\mathrm{G} / \mathrm{H}^{\prime}$ a un système sphérique qui s'obtient par induction parabolique à partir de $a z(3,3)$, ce qui n'est pas possible pour $a z^{\sim}(3+1+3)$ (en fait, $\mathrm{H}_{25}$ est magnifique, mais le système sphérique de $\mathrm{G} / \mathrm{H}_{25}$ diffère de $a z^{\sim}(3+1+3)$ en $\Delta\left(\alpha_{4}\right)$, qui contient un élément projectif), et $\mathrm{H}_{34}$ est conjugué à $\mathrm{H}_{25}$. Par suite $\mathrm{H}$ est égal à $\mathrm{H}_{35}$ ou à $\mathrm{H}_{24}$, qui sont conjugués.

(19) $a z^{\sim}(3+q+2), q \geqslant 1$

L'ensemble $\Delta$ est ici composé de 8 éléments : de $\mathrm{D}_{\alpha_{1}}^{+}=\mathrm{D}_{\alpha_{3}}^{+}=\mathrm{D}_{\alpha_{q+5}}^{+}$, de $\mathrm{D}_{\alpha_{2}}^{+}=\mathrm{D}_{\alpha_{q+4}}^{+}$, de $\mathrm{D}_{\alpha_{2}}^{-}=\mathrm{D}_{\alpha_{q+5}}^{-}$, de $\mathrm{D}_{\alpha_{1}}^{-}, \mathrm{D}_{\alpha_{3}}^{-}$et $\mathrm{D}_{\alpha_{q+4}}^{-}$; et si $q>1$, de $\mathrm{D}_{\alpha_{4}}$ et $\mathrm{D}_{\alpha_{q+3}}$ (ou si $q=1$, de $\mathrm{D}_{\alpha_{4}}^{+}$et $\mathrm{D}_{\alpha_{4}}^{-}$; pour distinguer les deux, on convient que $\left.<\rho\left(\mathrm{D}_{\alpha_{4}}^{-}\right), \alpha_{3}>=-1\right)$.

Posons $\Delta^{\prime}=\left\{\mathrm{D}_{\alpha_{1}}^{+}, \mathrm{D}_{\alpha_{2}}^{+}\right\}$. L'ensemble $\Delta^{\prime}$ est distingué et le triplet quotient $\left(\mathrm{S}^{p}, \Sigma, \mathbf{A}\right) / \Delta^{\prime}$ est :

$$
\begin{aligned}
& \mathrm{S}^{p} / \Delta^{\prime}=\left\{\alpha_{5}, \ldots, \alpha_{q+2}\right\} \\
& \Sigma / \Delta^{\prime}=\left\{\alpha_{2}+\alpha_{q+5}, \alpha_{4}+\ldots+\alpha_{q+3}\right\}
\end{aligned}
$$

$\mathbf{A} / \Delta^{\prime}=\varnothing$ si $q>1$ (si $q=1, \mathbf{A} / \Delta^{\prime}$ est concentré en $\alpha_{4}$, et sans élément projectif).

On a $\operatorname{dim} \mathrm{C}=2, \operatorname{dim} \mathrm{C}_{1}=4$ et $\operatorname{dim} \mathrm{H}_{1}^{u} / \mathrm{H}^{u}=2\left(=\operatorname{card} \Delta^{\prime}\right)$. Le $\mathrm{L}_{1}$-module $\mathrm{LieH}_{1}^{u}$ se décompose en 10 sous-modules irréductibles $\mathrm{W}_{i}(i=1, \ldots, 10)$. Rangeons ces sousmodules de façon à ce que les cinq premiers engendrent $\mathrm{LieH}_{1}^{u}$ comme algèbre de Lie, et que leurs poids sous $\mathrm{G}_{1}$ soient donnés par : $\delta_{1}=\alpha_{1}, \delta_{2}=\alpha_{3}, \delta_{3}=\alpha_{3}+\alpha_{4}$, $\delta_{4}=\alpha_{4}+\alpha_{q+4}$ et $\delta_{5}=\alpha_{q+4}$. Alors les $\mathrm{W}_{i}(i=1,2,4)$ sont de dimension 2 et les deux autres de dimension $2 q$.

Examinons d'abord le cas où $q>1$. Alors les $\mathrm{W}_{i}(i=1,2,4)$ sont isomorphes en tant que $\left(\mathrm{L}_{1}, \mathrm{~L}_{1}\right)$-module. De ce qui précède, on déduit que $\mathrm{C}=\left\{c \in \mathrm{C}_{1}\right.$, $\left.\delta_{1}(c)=\delta_{2}(c)=\delta_{4}(c)\right\}$, et que $\mathrm{LieH}^{u}$ est un sous- $\left(\mathrm{L}_{1}, \mathrm{~L}_{1}\right)$-module de codimension 2 dans $\mathrm{LieH}_{1}^{u}$, contenant les $\mathrm{W}_{i}(i \neq 1,2,4)$, mais ne contenant aucun des $\mathrm{W}_{i}(i=1,2,4)$, ce qui détermine $\mathrm{H}$ à conjugaison près.

Lorsque $q=1$, les $\mathrm{W}_{i}(i=1, \ldots, 5)$ sont isomorphes en tant que $\left(\mathrm{L}_{1}, \mathrm{~L}_{1}\right)$-modules. Pour tout couple $(i, j)(1 \leqslant i<j \leqslant 5)$ notons $\mathrm{H}_{i j}=\left(\mathrm{L}_{1}, \mathrm{~L}_{1}\right) \mathrm{C}_{i j} \mathrm{H}_{i j}^{u}$ le sous-groupe de $\mathrm{G}$, où $\mathrm{C}_{i j}$ est défini par $\left\{c \in \mathrm{C}_{1}, \boldsymbol{\delta}_{r}(c)=\delta_{s}(c)\right.$, pour $\left.r, s \in\{1, \ldots, 5\} \backslash\{i, j\}\right\}$, et où $\mathrm{LieH}_{i j}^{u}$ est un sous-( $\left.\mathrm{L}_{1}, \mathrm{~L}_{1}\right)$-module de codimension 2 dans $\mathrm{LieH}_{1}^{u}$, contenant les $\mathrm{W}_{k}(k>5), \mathrm{W}_{i}$ et $\mathrm{W}_{j}$, mais ne contenant aucun des autres $\mathrm{W}_{k}$. Le groupe $\mathrm{H}$ est conjugué à l'un des $\mathrm{H}_{i j}$. $\mathrm{Si}$ $i=1$, ou si $(i, j)=(2,3)$ ou $(4,5)$, alors $\mathrm{H}_{i j}$ contient le radical d'un parabolique, ce qui 
est exclu. Le groupe $\mathrm{H}_{25}$ est exclu, car il est contenu dans un sous-groupe magnifique $\mathrm{H}^{\prime}$, dont l'espace homogène $\mathrm{G} / \mathrm{H}^{\prime}$ a un système sphérique qui s'obtient par induction parabolique à partir de $a z(3,2)$, ce qui n'est pas possible pour $a z^{\sim}(3+1+2)$ (en fait, $\mathrm{H}_{25}$ est magnifique, mais le système sphérique de $\mathrm{G} / \mathrm{H}_{25}$ diffère de $a z^{\sim}(3+1+2)$ en $\Delta\left(\alpha_{4}\right)$, qui contient un élément projectif), et $\mathrm{H}_{34}$ est conjugué à $\mathrm{H}_{25}$. Par suite, $\mathrm{H}$ est égal à $\mathrm{H}_{35}$ ou à $\mathrm{H}_{24}$, qui sont conjugués.

(20) $a z(3,3)$

L'ensemble $\Delta$ est ici composé de 7 éléments : de $\mathrm{D}_{\alpha_{1}}^{+}=\mathrm{D}_{\alpha_{3}}^{+}=\mathrm{D}_{\beta_{2}}^{+}$, de $\mathrm{D}_{\alpha_{2}}^{+}=\mathrm{D}_{\beta_{1}}^{+}=\mathrm{D}_{\beta_{3}}^{+}$, de $\mathrm{D}_{\alpha_{2}}^{-}=\mathrm{D}_{\beta_{2}}^{-}$, de $\mathrm{D}_{\alpha_{1}}^{-}$, $\mathrm{D}_{\alpha_{3}}^{-}$et $\mathrm{D}_{\beta_{1}}^{-}$et de $\mathrm{D}_{\beta_{3}}^{-}$.

Posons $\Delta^{\prime}=\left\{\mathrm{D}_{\alpha_{1}}^{+}, \mathrm{D}_{\alpha_{2}}^{+}\right\}$. L'ensemble $\Delta^{\prime}$ est distingué et le triplet quotient $\left(\mathrm{S}^{p}, \Sigma, \mathbf{A}\right) / \Delta^{\prime}$ est :

$$
\begin{aligned}
& S^{p} / \Delta^{\prime}=\varnothing \\
& \Sigma / \Delta^{\prime}=\left\{\alpha_{2}+\beta_{2}\right\} \\
& \mathbf{A} / \Delta^{\prime}=\varnothing .
\end{aligned}
$$

On a $\operatorname{dim} \mathrm{C}=1, \operatorname{dim} \mathrm{C}_{1}=4$ et $\operatorname{dim} \mathrm{H}_{1}^{u} / \mathrm{H}^{u}=2\left(=\operatorname{card} \Delta^{\prime}\right)$. Le $\mathrm{L}_{1}$-module $\mathrm{LieH}_{1}^{u}$ se décompose en 6 sous-modules irréductibles $\mathrm{W}_{i}(i=1, \ldots, 6)$. Rangeons ces sous-modules de façon à ce que les quatre premiers engendrent $\mathrm{LieH}_{1}^{u}$ comme algèbre de Lie, et que leurs poids sous $\mathrm{C}_{1}$ soient donnés par : $\delta_{1}=\alpha_{1}, \delta_{2}=\alpha_{3}, \delta_{3}=\beta_{1}, \delta_{4}=\beta_{3}$. Alors les $\mathrm{W}_{i}(i=1,2,3,4)$ sont de dimension 2 et les deux autres de dimension 1. Les $\mathrm{W}_{i}(i=1,2,3,4)$ sont isomorphes en tant que $\left(\mathrm{L}_{1}, \mathrm{~L}_{1}\right)$-modules. De ce qui précède, on déduit que $\mathrm{C}=\left\{c \in \mathrm{C}_{1}, \delta_{1}(c)=\delta_{2}(c)=\delta_{3}(c)=\delta_{4}(c)\right\}$, et que $\mathrm{LieH}^{u}$ est un sous- $\left(\mathrm{L}_{1}, \mathrm{~L}_{1}\right)$ module de codimension 2 dans $\mathrm{LieH}_{1}^{u}$, contenant $\mathrm{W}_{5}$ et $\mathrm{W}_{6}$, mais ne contenant aucun des $\mathrm{W}_{i}(i=1,2,3,4)$, ce qui détermine $\mathrm{H}$ à conjugaison près.

(21) $a z(3,2)$

L'ensemble $\Delta$ est ici composé de 6 éléments : de $\mathrm{D}_{\alpha_{1}}^{+}=\mathrm{D}_{\alpha_{3}}^{+}=\mathrm{D}_{\beta_{2}}^{+}$, de $\mathrm{D}_{\alpha_{2}}^{+}=\mathrm{D}_{\beta_{1}}^{+}$, de $\mathrm{D}_{\alpha_{2}}^{-}=\mathrm{D}_{\beta_{2}}^{-}$, de $\mathrm{D}_{\alpha_{1}}^{-}, \mathrm{D}_{\alpha_{3}}^{-}$et $\mathrm{D}_{\beta_{1}}^{-}$.

Posons $\Delta^{\prime}=\left\{\mathrm{D}_{\alpha_{1}}^{+}, \mathrm{D}_{\alpha_{2}}^{+}\right\}$. L'ensemble $\Delta^{\prime}$ est distingué et le triplet quotient $\left(\mathrm{S}^{p}, \Sigma, \mathbf{A}\right) / \Delta^{\prime}$ est :

$$
\begin{aligned}
& \mathrm{S}^{p} / \Delta^{\prime}=\varnothing \\
& \Sigma / \Delta^{\prime}=\left\{\alpha_{2}+\beta_{2}\right\} \\
& \mathbf{A} / \Delta^{\prime}=\varnothing .
\end{aligned}
$$

On a $\operatorname{dim} \mathrm{C}=1, \operatorname{dim} \mathrm{C}_{1}=4$ et $\operatorname{dim}_{1}^{u} / \mathrm{H}^{u}=2\left(=\operatorname{card} \Delta^{\prime}\right)$. Le $\mathrm{L}_{1}$-module Lie $\mathrm{H}_{1}^{u}$ se décompose en 4 sous-modules irréductibles $\mathrm{W}_{i}(i=1,2,3,4)$. Rangeons ces sous-modules de façon à ce que les trois premiers engendrent $\mathrm{LieH}_{1}^{u}$ comme algèbre 
de Lie, et que leurs poids sous $\mathrm{C}_{1}$ soient donnés par : $\delta_{1}=\alpha_{1}, \delta_{2}=\alpha_{3}, \delta_{3}=\beta_{1}$. Alors les $\mathrm{W}_{i}(i=1,2,3)$ sont de dimension 2 et $\mathrm{W}_{4}$ est de dimension 1 . Les $\mathrm{W}_{i}$ $(i=1,2,3)$ sont isomorphes en tant que $\left(\mathrm{L}_{1}, \mathrm{~L}_{1}\right)$-modules. De ce qui précède, on déduit que $\mathrm{G}=\left\{c \in \mathrm{C}_{1}, \delta_{1}(c)=\delta_{2}(c)=\delta_{3}(c)\right\}$, et que $\mathrm{LieH}^{u}$ est un sous-( $\left.\mathrm{L}_{1}, \mathrm{~L}_{1}\right)$-module de codimension 2 dans $\mathrm{LieH}_{1}^{u}$, ne contenant aucun des $\mathrm{W}_{i}(i=1,2,3)$, ce qui détermine $\mathrm{H}$ à conjugaison près.

(22) $a z(3,1)$

L'ensemble $\Delta$ est ici composé de 5 éléments : de $\mathrm{D}_{\alpha_{1}}^{+}=\mathrm{D}_{\alpha_{3}}^{+}=\mathrm{D}_{\beta_{1}}^{+}, \mathrm{D}_{\alpha_{2}}^{+}$, de $\mathrm{D}_{\alpha_{2}}^{-}=\mathrm{D}_{\beta_{1}}^{-}$, de $\mathrm{D}_{\alpha_{1}}^{-}$et de $\mathrm{D}_{\alpha_{3}}^{-}$. Posons $\Delta^{\prime}=\left\{\mathrm{D}_{\alpha_{1}}^{+}, \mathrm{D}_{\alpha_{2}}^{+}\right\}$. L'ensemble $\Delta^{\prime}$ est distingué et le triplet quotient $\left(\mathrm{S}^{p}, \Sigma, \mathbf{A}\right) / \Delta^{\prime}$ est :

$$
\begin{aligned}
& S^{p} / \Delta^{\prime}=\varnothing \\
& \Sigma / \Delta^{\prime}=\left\{\alpha_{2}+\beta_{1}\right\} \\
& \mathbf{A} / \Delta^{\prime}=\varnothing .
\end{aligned}
$$

On a $\operatorname{dim} \mathrm{C}=1, \operatorname{dim} \mathrm{C}_{1}=2$ et $\operatorname{dim}_{1}^{u} / \mathrm{H}^{u}=2\left(=\operatorname{card} \Delta^{\prime}\right)$. Le $\mathrm{L}_{1}$-module $\mathrm{LieH}_{1}^{u}$ se décompose en 3 sous-modules irréductibles, dont l'un de dimension 1 et deux de dimension 2. Les deux de dimension 2 sont isomorphes comme $\left(\mathrm{L}_{1}, \mathrm{~L}_{1}\right)$-modules et leurs poids sous $\mathrm{G}_{1}$ sont $\alpha_{1}$ et $\alpha_{3}$. De ce qui précède suit que $\mathrm{G}=\left\{c \in \mathrm{C}_{1}, \alpha_{1}(c)=\alpha_{3}(c)\right\}$, et que $\mathrm{LieH}^{u}$ est un sous- $\left(\mathrm{L}_{1}, \mathrm{~L}_{1}\right)$-module de codimension 2 dans $\mathrm{LieH}_{1}^{u}$, non stable par $\mathrm{G}_{1}$, ce qui détermine $\mathrm{H}$ à conjugaison près.

(23) $a e_{6}(6)$

L'ensemble $\Delta$ est ici composé de 7 éléments : de $\mathrm{D}_{\alpha_{1}}^{+}=\mathrm{D}_{\alpha_{3}}^{+}=\mathrm{D}_{\alpha_{5}}^{+}$, de $\mathrm{D}_{\alpha_{2}}^{+}=\mathrm{D}_{\alpha_{4}}^{+}=\mathrm{D}_{\alpha_{6}}^{+}$, de $\mathrm{D}_{\alpha_{2}}^{-}=\mathrm{D}_{\alpha_{5}}^{-}$, et de $\mathrm{D}_{\alpha_{1}}^{-}, \mathrm{D}_{\alpha_{3}}^{-}, \mathrm{D}_{\alpha_{4}}^{-}$et $\mathrm{D}_{\alpha_{6}}^{-}$. Posons $\Delta^{\prime}=\left\{\mathrm{D}_{\alpha_{1}}^{+}, \mathrm{D}_{\alpha_{2}}^{+}\right\}$. L'ensemble $\Delta^{\prime}$ est distingué et le triplet quotient $\left(\mathbf{S}^{p}, \Sigma, \mathbf{A}\right) / \Delta^{\prime}$ est :

$$
\begin{aligned}
& S^{p} / \Delta^{\prime}=\varnothing \\
& \Sigma / \Delta^{\prime}=\left\{\alpha_{2}+\alpha_{5}\right\} \\
& \mathbf{A} / \Delta^{\prime}=\varnothing .
\end{aligned}
$$

On a $\operatorname{dim} \mathrm{C}=1, \operatorname{dim} \mathrm{C}_{1}=4$ et $\operatorname{dim}_{1}^{u} / \mathrm{H}^{u}=2\left(=\operatorname{card} \Delta^{\prime}\right)$. Le $\mathrm{L}_{1}$-module Lie $\mathrm{H}_{1}^{u}$ se décompose en 11 sous-modules irréductibles $\mathrm{W}_{i}(i=1, \ldots, 11)$. Rangeons ces sousmodules de façon à ce que les quatre premiers engendrent $\mathrm{LieH}_{1}^{u}$ comme algèbre de Lie, et que leurs poids sous $\mathrm{C}_{1}$ soient donnés par : $\delta_{1}=\alpha_{1}, \delta_{2}=\alpha_{3}, \delta_{3}=\alpha_{4}, \delta_{4}=\alpha_{6}$. Alors les $\mathrm{W}_{i}(i=1,2,3,4)$ sont de dimension 2 et sont isomorphes en tant que $\left(\mathrm{L}_{1}, \mathrm{~L}_{1}\right)$ modules. De ce qui précède, on déduit que $\mathrm{G}=\left\{c \in \mathrm{C}_{1}, \delta_{1}(c)=\delta_{2}(c)=\delta_{3}(c)=\delta_{4}(c)\right\}$, et que $\mathrm{LieH}^{u}$ est un sous- $\left(\mathrm{L}_{1}, \mathrm{~L}_{1}\right)$-module de codimension 2 dans $\mathrm{LieH}_{1}^{u}$, contenant les $\mathrm{W}_{i}(i \neq 1,2,3,4)$, mais ne contenant aucun des $\mathrm{W}_{i}(i=1,2,3,4)$, ce qui détermine $\mathrm{H}$ à conjugaison près. 
(24) $a e_{6}(5)$

L'ensemble $\Delta$ est ici composé de 6 éléments : de $\mathrm{D}_{\alpha_{1}}^{+}=\mathrm{D}_{\alpha_{3}}^{+}=\mathrm{D}_{\alpha_{5}}^{+}$, de $\mathrm{D}_{\alpha_{2}}^{+}=\mathrm{D}_{\alpha_{4}}^{+}$, de $\mathrm{D}_{\alpha_{2}}^{-}=\mathrm{D}_{\alpha_{5}}^{-}$, et de $\mathrm{D}_{\alpha_{1}}^{-}, \mathrm{D}_{\alpha_{2}}^{-}$et $\mathrm{D}_{\alpha_{3}}^{-}$.

Posons $\Delta^{\prime}=\left\{\mathrm{D}_{\alpha_{1}}^{+}, \mathrm{D}_{\alpha_{2}}^{+}\right\}$. L'ensemble $\Delta^{\prime}$ est distingué et le triplet quotient $\left(\mathrm{S}^{p}, \Sigma, \mathbf{A}\right) / \Delta^{\prime}$ est :

$$
\begin{aligned}
& \mathrm{S}^{p} / \Delta^{\prime}=\varnothing \\
& \Sigma / \Delta^{\prime}=\left\{\alpha_{2}+\alpha_{5}\right\} \\
& \mathbf{A} / \Delta^{\prime}=\varnothing .
\end{aligned}
$$

On a $\operatorname{dim} \mathrm{C}=1, \operatorname{dim} \mathrm{C}_{1}=3$ et $\operatorname{dim} \mathrm{H}_{1}^{u} / \mathrm{H}^{u}=2\left(=\operatorname{card} \Delta^{\prime}\right)$. Le $\mathrm{L}_{1}$-module Lie $\mathrm{H}_{1}^{u}$ se décompose en 7 sous-modules irréductibles $\mathrm{W}_{i}(i=1, \ldots, 7)$. Rangeons ces sous-modules de façon à ce que les trois premiers engendrent $\mathrm{LieH}_{1}^{u}$ comme algèbre de Lie, et que leurs poids sous $\mathrm{C}_{1}$ soient donnés par : $\delta_{1}=\alpha_{1}, \delta_{2}=\alpha_{3}, \delta_{3}=\alpha_{4}$. Alors les $\mathrm{W}_{i}$ $(i=1,2,3)$ sont de dimension 2 et sont isomorphes en tant que $\left(\mathrm{L}_{1}, \mathrm{~L}_{1}\right)$-modules. De ce qui précède, on déduit que $\mathrm{G}=\left\{c \in \mathrm{C}_{1}, \delta_{1}(c)=\delta_{2}(c)=\delta_{3}(c)\right\}$, et que Lie $\mathrm{H}^{u}$ est un sous- $\left(\mathrm{L}_{1}, \mathrm{~L}_{1}\right)$-module de codimension 2 dans $\mathrm{LieH}_{1}^{u}$, contenant les $\mathrm{W}_{i}(i \neq 1,2,3)$, mais ne contenant aucun des $\mathrm{W}_{i}(i=1,2,3)$, ce qui détermine $\mathrm{H}$ à conjugaison près.

(25) $a e_{6}(4)$

L'ensemble $\Delta$ est ici composé de 5 éléments : de $\mathrm{D}_{\alpha_{1}}^{+}=\mathrm{D}_{\alpha_{3}}^{+}$, de $\mathrm{D}_{\alpha_{2}}^{+}=\mathrm{D}_{\alpha_{4}}^{+}$, de $\mathrm{D}_{\alpha_{1}}^{-}=\mathrm{D}_{\alpha_{4}}^{-}$, et de $\mathrm{D}_{\alpha_{2}}^{-}$et $\mathrm{D}_{\alpha_{3}}^{-}$.

Posons $\Delta^{\prime}=\left\{\mathrm{D}_{\alpha_{1}}^{+}, \mathrm{D}_{\alpha_{2}}^{+}\right\}$. L'ensemble $\Delta^{\prime}$ est distingué et le triplet quotient $\left(\mathrm{S}^{p}, \Sigma, \mathbf{A}\right) / \Delta^{\prime}$ est :

$$
\begin{aligned}
& \mathrm{S}^{p} / \Delta^{\prime}=\varnothing \\
& \Sigma / \Delta^{\prime}=\left\{\alpha_{1}+\alpha_{4}\right\} \\
& \mathbf{A} / \Delta^{\prime}=\varnothing .
\end{aligned}
$$

On a $\operatorname{dimC}=1, \operatorname{dim}_{1}=2$ et $\operatorname{dimH}_{1}^{u} / \mathrm{H}^{u}=2\left(=\operatorname{card} \Delta^{\prime}\right)$. Le $\mathrm{L}_{1}$-module $\mathrm{LieH}_{1}^{u}$ se décompose en 4 sous-modules irréductibles dont deux de dimension 2 qui engendrent $\mathrm{LieH}_{1}^{u}$ comme algèbre de Lie, un de dimension 1 et un de dimension 3. Les deux sousmodules de dimension 2 sont isomorphes en tant que $\left(\mathrm{L}_{1}, \mathrm{~L}_{1}\right)$-modules et leurs poids sous $\mathrm{C}_{1}$ sont $\alpha_{2}$ et $\alpha_{3}$. De ce qui précède, on déduit que $\mathrm{C}=\left\{c \in \mathrm{C}_{1}, \alpha_{2}(c)=\alpha_{3}(c)\right\}$, et que $\mathrm{LieH}^{u}$ est un sous- $\left(\mathrm{L}_{1}, \mathrm{~L}_{1}\right)$-module de codimension 2 dans $\mathrm{LieH}_{1}^{u}$, non stable par $\mathrm{C}_{1}$, ce qui détermine $\mathrm{H}$ à conjugaison près.

(26) $a e_{7}(7)$

L'ensemble $\Delta$ est ici composé de 8 éléments : de $\mathrm{D}_{\alpha_{1}}^{+}=\mathrm{D}_{\alpha_{5}}^{+}$, de $\mathrm{D}_{\alpha_{2}}^{+}=\mathrm{D}_{\alpha_{4}}^{+}=\mathrm{D}_{\alpha_{6}}^{+}$, de $\mathrm{D}_{\alpha_{3}}^{+}=\mathrm{D}_{\alpha_{7}}^{+}$, de $\mathrm{D}_{\alpha_{2}}^{-}=\mathrm{D}_{\alpha_{5}}^{-}$, de $\mathrm{D}_{\alpha_{3}}^{-}=\mathrm{D}_{\alpha_{6}}^{-}$, et de $\mathrm{D}_{\alpha_{1}}^{-}, \mathrm{D}_{\alpha_{4}}^{-}$et $\mathrm{D}_{\alpha_{7}}^{-}$. 
Posons $\Delta^{\prime}=\left\{\mathrm{D}_{\alpha_{1}}^{+}, \mathrm{D}_{\alpha_{2}}^{+}, \mathrm{D}_{\alpha_{3}}^{+}\right\}$. L'ensemble $\Delta^{\prime}$ est distingué et le triplet quotient $\left(\mathrm{S}^{p}, \Sigma, \mathbf{A}\right) / \Delta^{\prime}$ est :

$$
\begin{aligned}
& \mathrm{S}^{p} / \Delta^{\prime}=\varnothing \\
& \Sigma / \Delta^{\prime}=\left\{\alpha_{2}+\alpha_{5}, \alpha_{3}+\alpha_{6}\right\} \\
& \mathbf{A} / \Delta^{\prime}=\varnothing .
\end{aligned}
$$

On a $\operatorname{dim} \mathrm{C}=1, \operatorname{dim} \mathrm{C}_{1}=3$ et $\operatorname{dim}_{1}^{u} / \mathrm{H}^{u}=3\left(=\operatorname{card} \Delta^{\prime}\right)$. Le $\mathrm{L}_{1}$-module Lie $\mathrm{H}_{1}^{u}$ se décompose en 7 sous-modules irréductibles $\mathrm{W}_{i}(i=1, \ldots, 7)$. Rangeons ces sous-modules de façon à ce que les quatre premiers engendrent $\mathrm{LieH}_{1}^{u}$ comme algèbre de Lie, que leurs poids sous $\mathrm{G}_{1}$ soient : $\delta_{1}=\alpha_{1}, \delta_{2}=\delta_{3}=\alpha_{4}, \delta_{4}=\alpha_{7}$, et que les $\mathrm{W}_{i}(i=1,2,4)$ soient de dimension 3 et $\mathrm{W}_{3}$ de dimension 6 . Alors les $\mathrm{W}_{i}$ $(i=1,2,4)$ sont isomorphes en tant que $\left(\mathrm{L}_{1}, \mathrm{~L}_{1}\right)$-modules. De ce qui précède, on déduit que $\mathrm{G}=\left\{c \in \mathrm{G}_{1}, \alpha_{1}(c)=\alpha_{4}(c)=\alpha_{7}(c)\right\}$, et que $\mathrm{LieH}^{u}$ est un sous-( $\left.\mathrm{L}_{1}, \mathrm{~L}_{1}\right)$-module de codimension 3 dans $\mathrm{LieH}_{1}^{u}$, contenant les $\mathrm{W}_{i}(i \neq 1,2,4)$, mais ne contenant aucun des $\mathrm{W}_{i}(i=1,2,4)$, ce qui détermine $\mathrm{H}$ à conjugaison près.

(27) $a e_{7}(6)$

L'ensemble $\Delta$ est ici composé de 7 éléments : de $\mathrm{D}_{\alpha_{1}}^{+}=\mathrm{D}_{\alpha_{5}}^{+}$, de $\mathrm{D}_{\alpha_{2}}^{+}=\mathrm{D}_{\alpha_{4}}^{+}=\mathrm{D}_{\alpha_{6}}^{+}$, de $\mathrm{D}_{\alpha_{2}}^{-}=\mathrm{D}_{\alpha_{5}}^{-}$, de $\mathrm{D}_{\alpha_{3}}^{-}=\mathrm{D}_{\alpha_{6}}^{-}$, et de $\mathrm{D}_{\alpha_{1}}^{-}, \mathrm{D}_{\alpha_{3}}^{+}$et $\mathrm{D}_{\alpha_{4}}^{-}$.

Posons $\Delta^{\prime}=\left\{\mathrm{D}_{\alpha_{1}}^{+}, \mathrm{D}_{\alpha_{2}}^{+}, \mathrm{D}_{\alpha_{3}}^{+}\right\}$. L'ensemble $\Delta^{\prime}$ est distingué et le triplet quotient $\left(\mathrm{S}^{p}, \Sigma, \mathbf{A}\right) / \Delta^{\prime}$ est :

$$
\begin{aligned}
& \mathrm{S}^{p} / \Delta^{\prime}=\varnothing \\
& \Sigma / \Delta^{\prime}=\left\{\alpha_{2}+\alpha_{5}, \alpha_{3}+\alpha_{6}\right\} \\
& \mathbf{A} / \Delta^{\prime}=\varnothing .
\end{aligned}
$$

On a $\operatorname{dim} \mathrm{C}=1, \operatorname{dim} \mathrm{C}_{1}=2$ et $\operatorname{dim} \mathrm{H}_{1}^{u} / \mathrm{H}^{u}=3\left(=\operatorname{card} \Delta^{\prime}\right)$. Le $\mathrm{L}_{1}$-module $\mathrm{LieH}_{1}^{u}$ se décompose en 4 sous-modules irréductibles $\mathrm{W}_{i}(i=1,2,3,4)$. Rangeons ces sousmodules de façon à ce que les quatre premiers engendrent Lie $\mathrm{H}_{1}^{u}$ comme algèbre de Lie, que leurs poids sous $\mathrm{C}_{1}$ soient $: \delta_{1}=\alpha_{1}, \delta_{2}=\delta_{3}=\alpha_{4}$, et que les $\mathrm{W}_{i}(i=1,2)$ soient de dimension 3 et $\mathrm{W}_{3}$ de dimension 6. Alors les $\mathrm{W}_{i}(i=1,2)$ sont isomorphes en tant que $\left(\mathrm{L}_{1}, \mathrm{~L}_{1}\right)$-modules. De ce qui précède, on déduit que $\mathrm{G}=\left\{c \in \mathrm{G}_{1}, \alpha_{1}(c)=\alpha_{4}(c)\right\}$, et que $\mathrm{LieH}^{u}$ est un sous- $\left(\mathrm{L}_{1}, \mathrm{~L}_{1}\right)$-module de codimension 3 dans $\mathrm{LieH}_{1}^{u}$, contenant les $\mathrm{W}_{i}$ $(i=3,4)$, mais ne contenant aucun des $\mathrm{W}_{i}(i=1,2)$, ce qui détermine $\mathrm{H}$ à conjugaison près. 
(28) $a e_{7}(5)$

L'ensemble $\Delta$ est ici composé de 6 éléments : de $\mathrm{D}_{\alpha_{1}}^{+}=\mathrm{D}_{\alpha_{3}}^{+}=\mathrm{D}_{\alpha_{5}}^{+}$, de $\mathrm{D}_{\alpha_{1}}^{-}=\mathrm{D}_{\alpha_{4}}^{-}$, de $\mathrm{D}_{\alpha_{2}}^{-}=\mathrm{D}_{\alpha_{5}}^{-}$, et de $\mathrm{D}_{\alpha_{2}}^{+}, \mathrm{D}_{\alpha_{3}}^{-}$et $\mathrm{D}_{\alpha_{4}}^{+}$.

Posons $\Delta^{\prime}=\left\{\mathrm{D}_{\alpha_{1}}^{+}, \mathrm{D}_{\alpha_{2}}^{+}, \mathrm{D}_{\alpha_{4}}^{+}\right\}$. L'ensemble $\Delta^{\prime}$ est distingué et le triplet quotient $\left(\mathrm{S}^{p}, \Sigma, \mathbf{A}\right) / \Delta^{\prime}$ est :

$$
\begin{aligned}
& \mathrm{S}^{p} / \Delta^{\prime}=\varnothing \\
& \Sigma / \Delta^{\prime}=\left\{\alpha_{1}+\alpha_{4}, \alpha_{2}+\alpha_{5}\right\} \\
& \mathbf{A} / \Delta^{\prime}=\varnothing .
\end{aligned}
$$

On a $\operatorname{dim} \mathrm{C}=\operatorname{dim} \mathrm{C}_{1}=1$ et $\operatorname{dimH}_{1}^{u} / \mathrm{H}^{u}=3\left(=\operatorname{card} \Delta^{\prime}\right)$. Par suite $\mathrm{L}=\mathrm{L}_{1}$. Comme le $\mathrm{L}_{1}$-module $\mathrm{LieH}_{1}^{u}$ se décompose en deux sous-modules irréductibles, l'un de dimension 3 et l'autre de dimension $6 . \mathrm{LieH}^{u}$ doit être celui de dimension 6 , ce qui détermine $\mathrm{H}$ à conjugaison près.

(29) af (4)

L'ensemble $\Delta$ est ici composé de 6 éléments : de $\mathrm{D}_{\alpha_{1}}^{+}=\mathrm{D}_{\alpha_{3}}^{+}$, de $\mathrm{D}_{\alpha_{2}}^{+}=\mathrm{D}_{\alpha_{4}}^{+}$, de $\mathrm{D}_{\alpha_{1}}^{-}, \mathrm{D}_{\alpha_{2}}^{-}, \mathrm{D}_{\alpha_{3}}^{-}$et $\mathrm{D}_{\alpha_{4}}^{-}$.

Posons $\Delta^{\prime}=\left\{\mathrm{D}_{\alpha_{1}}^{+}, \mathrm{D}_{\alpha_{2}}^{+}\right\}$. L'ensemble $\Delta^{\prime}$ est distingué et le triplet quotient $\left(\mathrm{S}^{p}, \Sigma, \mathbf{A}\right) / \Delta^{\prime}$ est :

$$
\begin{aligned}
& \mathrm{S}^{p} / \Delta^{\prime}=\varnothing \\
& \Sigma / \Delta^{\prime}=\left\{\alpha_{2}+\alpha_{3}\right\} \\
& \mathbf{A} / \Delta^{\prime}=\varnothing .
\end{aligned}
$$

On a $\operatorname{dim} \mathrm{C}=2, \operatorname{dim} \mathrm{C}_{1}=3$ et $\operatorname{dim}_{1}^{u} / \mathrm{H}^{u}=2\left(=\operatorname{card} \Delta^{\prime}\right)$. Le $\mathrm{L}_{1}$-module $\mathrm{LieH}_{1}^{u}$ se décompose en 5 sous-modules irréductibles $\mathrm{W}_{i}(i=1, \ldots, 5)$, deux de dimension 2 et trois de dimension 1. Rangeons ces sous-modules de façon à ce que $W_{1}$ et $W_{2}$ soient de dimension 2 , et tels que $\left[\mathrm{W}_{1}, \mathrm{~W}_{2}\right]=\left[\mathrm{W}_{3}, \mathrm{~W}_{4}\right]=\mathrm{W}_{5}$. Alors les $\mathrm{W}_{i}(i=1,2,4)$ sont isomorphes en tant que $\left(\mathrm{L}_{1}, \mathrm{~L}_{1}\right)$-modules et leurs poids sous $\mathrm{G}_{1}$ sont $: \delta_{1}=\alpha_{1}+\alpha_{2}$ et $\delta_{2}=\alpha_{4}$. De ce qui précède, on déduit que $\mathrm{C}=\left\{c \in \mathrm{C}_{1}, \delta_{1}(c)=\delta_{2}(c)\right\}$, et que $\mathrm{LieH}^{u}$ est un sous- $\left(\mathrm{L}_{1}, \mathrm{~L}_{1}\right)$-module de codimension 2 dans $\mathrm{LieH}_{1}^{u}$, contenant les $\mathrm{W}_{i}(i=3,4,5)$, mais ne contenant aucun des $\mathrm{W}_{i}(i=1,2)$, ce qui détermine $\mathrm{H}$ à conjugaison près.

\subsection{Les cas primitifs non classiques : «l'existence»}

Montrons maintenant l'existence de réalisations géométriques pour les systèmes sphériques primitifs non classiques. On peut laisser de côté les cas qui se déduisent par localisation à partir d'autres cas primitifs, ce qui laisse neuf cas. Pour chacun des cas, la partie «unicité» de la preuve fournit un candidat $\mathrm{H}=\left(\mathrm{L}_{1}, \mathrm{~L}_{1}\right) \mathrm{CH}^{u}$. 
Rappelons que :

1) $H_{1}$ est un sous-groupe magnifique connexe d'un groupe adjoint $G$, ayant une décomposition de Levi $\mathrm{H}_{1}=\mathrm{L}_{1} \mathrm{H}_{1}^{u}$ telle que $\mathrm{H}_{1}^{u}$ est le radical unipotent d'un sous-groupe parabolique de $\mathrm{G}$;

2) $\mathrm{H}$ est un sous-groupe de $\mathrm{H}_{1}$ ayant une décomposition de Levi $\mathrm{H}=\mathrm{LH}^{u}$, vérifiant : $\mathrm{L} \subset \mathrm{L}_{1},(\mathrm{~L}, \mathrm{~L})=\left(\mathrm{L}_{1}, \mathrm{~L}_{1}\right)$ (et donc $\left.\mathrm{H}^{u} \subset \mathrm{H}_{1}^{u}\right)$;

3) $\mathrm{G}$ et $\mathrm{C}_{1}$ désignent les radicaux (les centres connexes) de $\mathrm{L}$ et $\mathrm{L}_{1}$.

Voici deux résultats auxiliaires qu'on utilisera plusieurs fois.

(*) Désignons par $Q$ le sous-groupe parabolique $\mathrm{N}_{\mathrm{G}}\left(\mathrm{H}_{1}^{u}\right)$ et par $\mathrm{L}(\mathrm{Q})$ un sousgroupe de Levi de $\mathrm{Q}$ contenant $\mathrm{L}_{1}$. Pour que $\mathrm{H}$ soit sphérique dans $\mathrm{G}$, il faut et il suffit qu'il existe un sous-groupe de Borel $\mathrm{B}^{\prime}$ de $\mathrm{L}(\mathrm{Q})$ vérifiant :

a) $\mathrm{B}^{\prime} \mathrm{L}$ est ouvert dans $\mathrm{L}(\mathrm{Q})$;

b) $\mathrm{B}^{\prime} \cap \mathrm{L}$ a une orbite ouverte dans $\mathrm{LieH}_{1}^{u} / \mathrm{LieH}^{u}$.

(**) On suppose que $\mathrm{H}$ est magnifique, et qu'il existe une composante $\Delta$-connexe isolée $\Sigma^{\prime}$ de $\Sigma_{\mathrm{G} / \mathrm{H}_{1}}$ telle que le localisé du système sphérique de $\mathrm{G} / \mathrm{H}_{1}$ en $\operatorname{supp}\left(\Sigma^{\prime}\right)$ soit isomorphe à $a a(p+q+p)(p \geqslant 2, q \geqslant 1)$ où à $a a(q)(q \geqslant 1)$. Notons $\gamma$ l'unique racine de type $a_{q}$ dans $\Sigma^{\prime}$. La décomposition $\mathrm{S}=\operatorname{supp}\left(\Sigma^{\prime}\right) \cup\left(\mathrm{S} \backslash \operatorname{supp}\left(\Sigma^{\prime}\right)\right)$ induit une décomposition $\left(\mathrm{L}_{1}, \mathrm{~L}_{1}\right)=\mathrm{K}^{\prime} \times \mathrm{K}^{\prime \prime}$, où $\mathrm{K}^{\prime} \cong \mathrm{SL}_{p+1}(\mathbf{G}) \times \mathrm{SL}_{p+q}(\mathbf{C})$; si $q \geqslant 2$, notons $\mathrm{K}$ le facteur de $\mathrm{K}^{\prime}$ correspondant à $\mathrm{SL}_{p+q}(\mathbf{G})$. Alors,

1) si $q \geqslant 2$, et si $\mathrm{K}$ opère trivialement dans $\mathrm{LieH}_{1}^{u} / \mathrm{Lie} \mathrm{H}^{u}$, alors $\gamma \in \Sigma_{\mathrm{G} / \mathrm{H}}$;

2) si $q=1$, alors $\gamma \in \Sigma_{\mathrm{G} / \mathrm{H}}$, et $\Delta_{\mathrm{G} / \mathrm{H}}(\gamma) \cap \Delta_{\mathrm{G} / \mathrm{H}}(\alpha)=\varnothing$ quel que soit $\alpha \in \mathrm{S} \backslash\{\gamma\}$.

Preuve de (*) (voir aussi [Br2])

Désignons par $Q_{-}$le sous-groupe parabolique de $G$ opposé à $Q$ tel que $\mathrm{Q} \cap \mathrm{Q}_{-}=\mathrm{L}(\mathrm{Q})$. L'assertion $\left(^{*}\right)$ résulte aussitôt de la suite exacte

$$
1 \rightarrow \mathrm{H}_{1}^{u} / \mathrm{H}^{u} \rightarrow \mathrm{Q} / \mathrm{H} \rightarrow \mathrm{L}(\mathrm{Q}) / \mathrm{L} \rightarrow 1,
$$

du fait que $Q_{-}^{u} \mathrm{Q} / \mathrm{H}$ est ouvert dans $\mathrm{G} / \mathrm{H}$, et de ce que $\mathrm{B}=\mathrm{B}^{\prime} \mathrm{Q}_{-}^{u}$ est un sous-groupe de Borel de $G$, quel que soit le sous-groupe de Borel $B^{\prime}$ de $L(Q)$.

\section{Preuve de (**)}

Posons $\operatorname{supp}\left(\Sigma^{\prime}\right)=\left\{\beta_{1}, \ldots, \beta_{n}\right\}$ avec $n=2 p+q$; c'est la base d'un système de racines de type $\mathrm{A}_{n}$, qu'on ordonne comme d'habitude. On a $\gamma=\beta_{p+1}+\ldots+\beta_{p+q}$. Posons $\mathrm{S}^{\prime}=\left\{\beta_{p^{+1}}, \ldots, \beta_{p+q}\right\}$, et $\mathrm{S}^{\prime \prime}=\left\{\beta_{p^{+2}}, \ldots, \beta_{p^{+q-1}}\right\}$.

Soit $B$ un sous-groupe de Borel de $G$ contenant $Q_{-}^{u}$. On pose $B^{\prime}=B \cap L(Q)$, et on suppose que $\mathrm{B}^{\prime} \mathrm{L}$ est ouvert dans $\mathrm{L}(\mathrm{Q})$. Pour tout $\alpha \in \mathrm{S}$, notons $\mathrm{G}_{\{\alpha\}}$ le sous-groupe parabolique de $\mathrm{G}$ contenant $\mathrm{B}$ et associé à $\alpha$. 
Lemme. - Mêmes hypothèses que pour (**). Si $q \geqslant 3$ et $\alpha \in \mathrm{S}^{\prime \prime}$ (ou si $q=2$ et $\alpha \in \mathrm{S}^{\prime}$ ), il existe un sous-groupe $\mathrm{K}_{\alpha}$ de $\mathrm{K}$ tel que $\mathrm{G}_{\{\alpha\}} \cap \mathrm{L}=(\mathrm{B} \cap \mathrm{L}) \mathrm{K}_{\alpha}$.

Preuve du lemme. - On se ramène aussitôt au cas où $\mathrm{S}=\mathrm{S}^{\prime}$, c'est-à-dire au cas où $\mathrm{G}=\mathrm{SL}_{n}(\mathbf{C})$ et où $\mathrm{L}_{1}=\mathrm{H}_{1}=\mathrm{N}_{\mathrm{G}}\left(\mathrm{SL}_{p+1}(\mathbf{C}) \times \mathrm{SL}_{p+q}(\mathbf{G})\right)$. Soit $\mathrm{B}$ un sous-groupe de Borel de $\mathrm{G}$ tel que $\mathrm{BL}_{1}$ est ouvert dans $\mathrm{G}$. Comme $\left(\mathrm{L}_{1}, \mathrm{~L}_{1}\right) \subset \mathrm{L} \subset \mathrm{L}_{1}$, BL est alors aussi ouvert dans $\mathrm{G}$. Lorsque $q \geqslant 3$ et $\alpha \in \mathrm{S}^{\prime \prime}$ (resp. lorsque $q=2$ et $\alpha \in \mathrm{S}^{\prime}$ ), on vérifie qu'il existe un sous-groupe $\mathrm{K}_{\alpha}$ semi-simple de dimension 3 (resp. unipotent de dimension 1) dans $\left.\mathrm{K}=\mathrm{SL}_{p+q}(\mathbf{G})\right)$ tel que $\mathrm{G}_{\{\alpha\}} \cap \mathrm{L}_{1}=\left(\mathrm{B} \cap \mathrm{L}_{1}\right) \mathrm{K}_{\alpha}$. Il s'ensuit qu'on a également $\mathrm{G}_{\{\alpha\}} \cap \mathrm{L}=(\mathrm{B} \cap \mathrm{L}) \mathrm{K}_{\alpha}$.

Retournons à la preuve de (**).

Supposons $q \geqslant 3$. D'après le lemme, pour tout $\alpha \in \mathrm{S}^{\prime \prime}$, le groupe $\mathrm{G}_{\{\alpha\}} \cap \mathrm{L}$ laisse stable l'orbite ouverte de $\mathrm{B} \cap \mathrm{L}$ dans $\mathrm{H}_{1}^{u} / \mathrm{H}^{u}$. D'autre part, par hypothèse, $\mathrm{G}_{\{\alpha\}} \cap \mathrm{L}(\mathrm{Q})$ laisse stable l'orbite ouverte de $\mathrm{B}^{\prime}$ dans $\mathrm{L}(\mathrm{Q}) / \mathrm{L}_{1}$, donc aussi l'orbite ouverte de $\mathrm{B}^{\prime}$ dans $\mathrm{L}(\mathrm{Q}) / \mathrm{L}$. De la suite exacte

$$
1 \rightarrow \mathrm{H}_{1}^{\mathrm{u}} / \mathrm{H}^{\mathrm{u}} \rightarrow \mathrm{Q} / \mathrm{H} \rightarrow \mathrm{L}(\mathrm{Q}) / \mathrm{L} \rightarrow 1
$$

et du fait que $\mathrm{Q}^{u} \mathrm{Q} / \mathrm{H}$ est ouvert dans $\mathrm{G} / \mathrm{H}$, il s'ensuit que $\mathrm{G}_{\{\alpha\}}$ laisse stable l'orbite ouverte de $\mathrm{B}$ dans $\mathrm{G} / \mathrm{H}$, ce qui implique $\alpha \in \mathrm{S}_{\mathrm{G} / \mathrm{H}}^{p}$.

Supposons $q=2$. D'après le lemme, pour tout $\alpha \in \mathrm{S}^{\prime}$, le groupe $\mathrm{G}_{\{\alpha\}} \cap \mathrm{L}$ laisse stable l'orbite ouverte de $\mathrm{B} \cap \mathrm{L}$ dans $\mathrm{H}_{1}^{u} / \mathrm{H}^{u}$. Comme ci-dessus, on en déduit que $\mathrm{B}$ a même nombre d'orbites dans l'orbite ouverte de $\mathrm{G}_{\{\alpha\}}$ dans $\mathrm{G} / \mathrm{H}$ que dans l'orbite ouverte de $\mathrm{G}_{\{\alpha\}}$ dans $\mathrm{G} / \mathrm{H}_{1}$, c'est-à-dire deux, ce qui implique $\alpha \in \mathrm{S}_{\mathrm{G} / \mathrm{H}}^{b} \cup \mathrm{S}_{\mathrm{G} / \mathrm{H}}^{a^{\prime}}$.

Notons $\mathrm{X}$ et $\mathrm{X}_{1}$ les variétés magnifiques de $\mathrm{G} / \mathrm{H}$ et $\mathrm{G} / \mathrm{H}_{1}$, et $\mathrm{X}^{\prime}$ et $\mathrm{X}_{1}^{\prime}$ les localisés de $\mathrm{X}$ et $\mathrm{X}_{1}$ en $\mathrm{S}^{\prime}$. La variété $\mathrm{X}_{1}^{\prime}$ a comme système sphérique $a a(q)$ avec racine sphérique $\gamma$. Le morphisme surjectif $\mathrm{X} \rightarrow \mathrm{X}_{1}$ induit un morphisme surjectif $\mathrm{X}^{\prime} \rightarrow \mathrm{X}_{1}^{\prime}$. De cela, et si $q \geqslant 3$, de $\mathrm{S}^{\prime \prime} \subset \mathrm{S}_{\mathrm{G} / \mathrm{H}}^{p}$ (et si $q=2$, de $\mathrm{S}^{\prime} \subset \mathrm{S}_{\mathrm{G} / \mathrm{H}}^{b} \cup \mathrm{S}_{\mathrm{G} / \mathrm{H}}^{a^{\prime}}$ ), on déduit que $\mathrm{X}^{\prime} \rightarrow \mathrm{X}_{1}^{\prime}$ est un isomorphisme, d'où $\gamma \in \Sigma_{\mathrm{X}^{\prime}} \subset \Sigma_{\mathrm{G} / \mathrm{H}}$. Enfin si $q=1$, le morphisme surjectif $\mathrm{X}^{\prime} \rightarrow \mathrm{X}_{1}^{\prime}$ est un isomorphisme (sans qu'on ait besoin d'hypothèse supplémentaire), donc $\gamma \in \Sigma_{\mathrm{G} / \mathrm{H}}$; de plus, comme $\Delta_{\mathrm{G} / \mathrm{H}_{1}}(\gamma) \cap \Delta_{\mathrm{G} / \mathrm{H}_{1}}(\alpha)=\varnothing$ quel que soit $\alpha \in \mathrm{S} \backslash\{\gamma\}$, de la surjectivité de $\mathrm{X} \rightarrow \mathrm{X}_{1}$ résulte que $\Delta_{\mathrm{G} / \mathrm{H}}(\gamma) \cap \Delta_{\mathrm{G} / \mathrm{H}}(\alpha)=\varnothing$ quel que soit $\alpha \in S \backslash\{\gamma\}$.

Voici l'argument type (assez indirect) qu'on utilisera pour montrer l'existence d'une réalisation géométrique pour les neuf systèmes en question. Soit $\mathrm{S}^{p}, \Sigma, \mathbf{A}$ l'un de ces systèmes, et soit $\mathrm{H}$ le candidat donné par la partie «unicité» de la preuve. On déduira de $\left(^{*}\right)$ que $\mathrm{H}$ est sphérique. On vérifiera cas par cas que $\mathrm{N}_{\mathrm{G}}(\mathrm{H})=\mathrm{H}$. Cela implique que $\mathrm{H}$ est un sous-groupe magnifique de $\mathrm{G}$ ([Kn2]). Des considérations de dimension de $\mathrm{H}$ et de $\mathrm{C}$ (et dans certains cas $(* *))$ permettront alors de montrer que 
$\Sigma_{\mathrm{G} / \mathrm{H}}=\Sigma$ et que $\mathrm{S}_{\mathrm{G} / \mathrm{H}}^{p}=\mathrm{S}^{p}$. Enfin, puisque la dimension de $\mathrm{C}$ sera égale à 1 ou 2 (dans les neuf cas considérés), il reste peu de possibilités pour $\mathbf{A}_{\mathrm{G} / \mathrm{H}}$, qu'on éliminera toutes, sauf $\mathbf{A}$.

(7) $a c^{*}(n), n \geqslant 3$

On peut supposer $n$ pair (le cas $n$ impair s'en déduit par localisation). Soient $G$ et $\mathrm{H}$ les groupes introduits dans la partie «unicité» de la preuve. Grâce à (*), on voit que $\mathrm{H}$ est sphérique dans $\mathrm{G}$. On vérifie que $\mathrm{N}_{\mathrm{G}}(\mathrm{H})=\mathrm{H}$ et que $\operatorname{dim} \mathrm{H}=\operatorname{dimB} \mathrm{B}^{u}+1$. Par suite, l'espace homogène $\mathrm{G} / \mathrm{H}$ possède un plongement magnifique, qui est cuspidal, car $\mathrm{H}$ n'est contenu que dans deux sous-groupes paraboliques propres, dont $\mathrm{H}$ ne contient pas le radical. De $\operatorname{rang} \Xi(\mathrm{H})=1$ suit que $\mathrm{G} / \mathrm{H}$ possède $n$ couleurs. Mais le seul système sphérique cuspidal de type $\mathrm{A}_{n}$, de dimension $\operatorname{dimB}^{u}+1$, et ayant $n$ couleurs, est le système $a c^{*}(n)$.

(8) $a x(1+p+1+q+1), n=p+q+3, p \geqslant 1, q \geqslant 1$

Soient $\mathrm{G}$ et $\mathrm{H}$ les groupes introduits dans la partie «unicité» de la preuve. De $\left(^{*}\right)$ on déduit que $H$ est sphérique dans $G$. On vérifie que $\mathrm{N}_{\mathrm{G}}(\mathrm{H})=\mathrm{H}$, et que le plongement magnifique de $\mathrm{G} / \mathrm{H}$ est cuspidal (car $\mathrm{H}$ n'est contenu dans aucun sousgroupe parabolique propre, dont le radical est contenu dans $\mathrm{H})$. De $(* *)$ on tire que $\alpha_{p+3}+\ldots+\alpha_{n-1} \in \Sigma_{\mathrm{G} / \mathrm{H}}$ et, si $q=1$, que $\Delta_{\mathrm{G} / \mathrm{H}}\left(\alpha_{p+3}\right) \cap \Delta_{\mathrm{G} / \mathrm{H}}\left(\alpha_{i}\right)=\varnothing$ pour tout $i \neq p+3$. Puisque $p$ et $q$ jouent un rôle symétrique, on a aussi $\alpha_{2}+\ldots+\alpha_{p+1} \in \Sigma_{\mathrm{G} / \mathrm{H}}$ et, si $p=1$, que $\Delta\left(\alpha_{2}\right) \cap \Delta\left(\alpha_{i}\right)=\varnothing$ pour tout $i \neq 2$. Pour des raisons de dimension, on voit que $\mathrm{S}_{\mathrm{G} / \mathrm{H}}^{p}=\mathrm{S}^{p}$ et que $\Sigma_{\mathrm{G} / \mathrm{H}}$ est composé de 5 racines sphériques. Puisque aucune ne peut être de type $a^{\prime}$, on a $\Sigma_{\mathrm{G} / \mathrm{H}}=\Sigma$. De $\operatorname{rang} \Xi(\mathrm{H})=2$ suit que $\mathrm{G} / \mathrm{H}$ possède 7 couleurs. On en déduit que le localisé en $\mathrm{S}^{\prime}=\left\{\alpha_{1}, \alpha_{p+1}, \alpha_{n}\right\}$ du système sphérique de $\mathrm{G} / \mathrm{H}$ est isomorphe à $a x(1,1,1)$. Si $p>1$ et $q>1, a x(1+p+1+q+1)$ est le seul système sphérique ayant toutes les propriétés ci-dessus. Si $p=1$, on doit considérer aussi la «variante» de $a x(1+p+1+q+1)$ obtenue en mettant un élément projectif dans $\Delta\left(\alpha_{2}\right)$. Mais si $\mathrm{G} / \mathrm{H}$ était une réalisation géométrique de cette variante, alors $\mathrm{H}$ serait contenu dans un sous-groupe parabolique $Q$ vérifiant $S_{\mathrm{G} / \mathrm{Q}}^{p}=\mathrm{S} \backslash\left\{\alpha_{2}\right\}$, ce qui n'est pas vrai. On règle de la même façon le cas $q=1$. Donc, dans tous les cas, $\mathrm{G} / \mathrm{H}$ est bien une réalisation géométrique de $a x(1+p+1+q+1)$.

On va maintenant traiter parallèlement les cas :

(12) $a y(p+q+p), n=2 p+q, p \geqslant 2, q \geqslant 1$

(16) $a y^{\sim}(p+q+p), n=2 p+q, p \geqslant 2, q \geqslant 1$

(17) $a y^{*}(2+q+2), n=4+q, p=2, q \geqslant 1$

(18) $a z^{\sim}(3+q+3), n=6+q, p=3, q \geqslant 1$

Dans chacun des quatre cas, soient $G$ et $H$ les groupes introduits dans la partie «unicité» de la preuve. De $\left(^{*}\right)$ suit que $\mathrm{H}$ est sphérique dans G. On 
vérifie que $\mathrm{N}_{\mathrm{G}}(\mathrm{L}) \cap \mathrm{N}_{\mathrm{G}}\left(\mathrm{H}^{u}\right)=\mathrm{L}$, d'où il suit que $\mathrm{N}_{\mathrm{G}}(\mathrm{H})=\mathrm{H}$. De (**) on déduit que $\alpha_{p+1}+\ldots+\alpha_{p+q} \in \Sigma_{\mathrm{G} / \mathrm{H}}$ et, si $q=1$, que $\Delta\left(\alpha_{p+1}\right) \cap \Delta\left(\alpha_{i}\right)=\varnothing$ pour tout $i \neq p+1$. Le plongement magnifique de $\mathrm{G} / \mathrm{H}$ est cuspidal (car $\mathrm{H}$ n'est contenu dans aucun sousgroupe parabolique propre, dont $\mathrm{H}$ contient le radical). Pour des raisons de dimension, on voit que $\mathrm{S}_{\mathrm{G} / \mathrm{H}}^{p}=\mathrm{S}^{p}$, et que $\Sigma_{\mathrm{G} / \mathrm{H}}$ est composé de $2 p+1$ racines sphériques, dont $2 p$ sont de type $a_{1}$ (car aucune ne peut être de type $a^{\prime}$ ). De rang $\Xi(\mathrm{H})=2$, il suit que $\mathrm{G} / \mathrm{H}$ possède $2 p+3$ couleurs. On en déduit que le localisé du système sphérique de $\mathrm{G} / \mathrm{H}$ en $\mathrm{S}^{\prime}=\left\{\alpha_{1}, \ldots, \alpha_{p}, \alpha_{p+q+1}, \ldots, \alpha_{n}\right\}$ ne contient que des racines sphériques de type $a_{1}$ et possède $2 p+1$ couleurs, donc est $\Delta$-connexe. Voici tous les systèmes sphériques qui possèdent ces propriétés:

1) les quatre systèmes sphériques considérés, et le système «dual» de $a y(p+q+p)$ (obtenu à partir de $a y(p+q+p)$ par un automorphisme extérieur);

2) si $q=1$, il y a aussi les variantes de ces systèmes, en mettant un élément projectif dans $\Delta\left(\alpha_{p+1}\right)$.

Dans la partie «unicité» de la preuve, on a montré que l'application, qui aux systèmes 1) et 2) associe la classe de conjugaison du candidat $\mathrm{H}$ correspondant, est injective. Il s'ensuit que, pour chacun des quatre systèmes considérés, G/H est bien une réalisation géométrique.

On va maintenant traiter parallèlement les cas :

(23) $a e_{6}(6)$

(27) $a e_{7}(7)$

Dans chacun des deux cas, soient $\mathrm{G}$ et $\mathrm{H}$ les groupes introduits dans la partie «unicité» de la preuve. De $\left(^{*}\right)$ suit que $\mathrm{H}$ est sphérique dans $\mathrm{G}$. On vérifie que $\mathrm{N}_{\mathrm{G}}(\mathrm{L}) \cap \mathrm{N}_{\mathrm{G}}\left(\mathrm{H}^{u}\right)=\mathrm{L}$, d'où il suit que $\mathrm{N}_{\mathrm{G}}(\mathrm{H})=\mathrm{H}$. Pour des raisons de dimension, $\mathrm{S}_{\mathrm{G} / \mathrm{H}}^{p}=\varnothing$ et $\Sigma_{\mathrm{G} / \mathrm{H}}$ contient card $(\mathrm{S})$ éléments. Aucune racine sphérique ne pouvant être de type $a^{\prime}$, on a $\Sigma_{\mathrm{G} / \mathrm{H}}=\mathrm{S}$. Comme $\operatorname{rang} \Xi(\mathrm{H})=1$, il suit que $\mathbf{A}_{\mathrm{G} / \mathrm{H}}$ contient $\operatorname{card}(\mathrm{S})+1$ éléments. Lorsque $\operatorname{card}(\mathrm{S})=7, a_{7}(7)$ est le seul système qui possède ces propriétés, donc $\mathrm{G} / \mathrm{H}$ est bien une réalisation géométrique. Lorsque $\operatorname{card}(\mathrm{S})=6$, il y en a deux, $a e_{6}(6)$ et $a e_{7}(6)$. D'après la partie «unicité» de la preuve, les candidats $\mathrm{H}$ de ces deux systèmes ne sont pas conjugués. Par conséquent, pour $a e_{6}(6)$ aussi, G/H est bien une réalisation géométrique.

(29) $a f(4)$

Soient $\mathrm{G}$ et $\mathrm{H}$ les groupes introduits dans la partie «unicité» de la preuve. De $\left(^{*}\right)$ suit que $H$ est sphérique dans $G$. On vérifie que $\mathrm{N}_{\mathrm{G}}(\mathrm{L}) \cap \mathrm{N}_{\mathrm{G}}\left(\mathrm{H}^{u}\right)=\mathrm{L}$, d'où $\mathrm{N}_{\mathrm{G}}(\mathrm{H})=\mathrm{H}$. Pour des raisons de dimension, $\mathrm{S}_{\mathrm{G} / \mathrm{H}}^{p}=\varnothing$ et $\Sigma_{\mathrm{G} / \mathrm{H}}$ contient quatre éléments. Aucune racine sphérique ne pouvant être de type $a^{\prime}$, on a $\Sigma_{\mathrm{G} / \mathrm{H}}=\mathrm{S}$. Comme rang $\Xi(\mathrm{H})=2$, 
il suit que $\mathbf{A}_{\mathrm{G} / \mathrm{H}}$ contient six éléments. Puisque $\mathrm{H}$ n'est pas résoluble, il n'y a pas de sous-ensemble distingué parabolique de $\Delta_{\mathrm{G} / \mathrm{H}}$ ayant deux éléments. Comme le système af(4) est le seul système sphérique possédant toutes ces propriétés, G/H est bien une réalisation géométrique de af(4).

Les autres systèmes sphériques primitifs non classiques sont obtenus par localisation à partir des neuf systèmes précédents.

\subsection{Réduction du cas général au cas primitif}

Soit $\mathrm{S}^{p}, \Sigma, \mathbf{A}$ un système sphérique (adjoint de type A). Montrons qu'il existe une variété magnifique unique (à isomorphisme près) ayant $\mathrm{S}^{p}, \Sigma, \mathbf{A}$ comme système sphérique.

On raisonne par récurrence sur le rang de $\mathrm{S}$. La proposition 3.4 ramène le cas général au cas cuspidal. La proposition 3.6 permet de supposer qu'il n'y a pas d'élément projectif (dans $\mathbf{A}$ ). D'après le $\S 4$, toute composante $\Delta$-connexe qui contient une racine sphérique de type $a^{\prime}, a_{1} \times a_{1}$ ou $d_{3}$ est alors isolée, et engendre un soussystème primitif (classique). Comme le théorème 1 est vrai pour ces systèmes (voir 5.2), on est ramené au cas où $\mathrm{S}^{p}, \Sigma, \mathbf{A}$ est un système cuspidal, sans couleur projective, et dont toutes les racines sphériques sont de type $a_{n}(n \geqslant 1)$, ce qu'on supposera désormais.

Supposons d'abord que $\mathrm{S}^{p}, \Sigma, \mathbf{A}$ contient plusieurs composantes $\Delta$-connexes effaçables. Désignons par $\Sigma_{1}, \Sigma_{2}$ deux de ces composantes, et posons $\Delta_{i}=\Delta\left(\Sigma_{i}\right)(i=1,2)$.

Montrons que le couple $\Delta_{1}, \Delta_{2}$ décompose le système sphérique $\mathrm{S}^{p}, \Sigma$, A. Par définition, les $\Delta_{1}, \Delta_{2}$ sont distingués et le couple possède les propriétés (1), (2), (3) et (5). Soit $\alpha_{i} \in \operatorname{supp}\left(\Sigma_{i}\right)(i=1,2)$. Si $\alpha_{1}$ n'est pas orthogonal à $\alpha_{2}$, comme toutes les racines sphériques sont de type $a_{n}(n \geqslant 1)$, on voit que $\Delta\left(\alpha_{1}\right) \not \subset \Delta_{1}$, ce qui implique (4).

La proposition 3.5 et l'hypothèse de récurrence ramènent alors au cas où $\mathrm{S}^{p}$, $\Sigma, \mathbf{A}$ ne contient qu'une seule composante $\Delta$-connexe effaçable. Le lemme suivant ou bien ramène au cas primitif, ou bien permet de raisonner de nouveau par récurrence grâce à la proposition 3.5.

Lemme - Soit $\mathrm{S}^{p}, \Sigma, \mathbf{A}$ un système cuspidal, sans couleur projective, dont toutes les racines sphériques sont de type $a_{n}(n \geqslant 1)$. On suppose qu'une seule composante $\Delta$-connexe $\Sigma_{1}$ de $\Sigma$ est effaçable. Posons $\Sigma_{2}=\Sigma \backslash \Sigma_{1}$, et $\Delta_{i}=\Delta\left(\Sigma_{i}\right)(i=1,2)$. Alors :

ou bien $\mathrm{S}^{p}, \Sigma, \mathbf{A}$ est primitif,

ou bien le couple $\Delta_{1}, \Delta_{2}$ décompose $\mathrm{S}^{p}, \Sigma, \mathbf{A}$.

Preuve - Si $\Sigma_{2}=\varnothing$, alors $\mathrm{S}^{p}, \Sigma, \mathbf{A}$ est primitif.

Si $\Sigma_{2} \neq \varnothing$ et $\Delta_{2}=\varnothing$, alors $\mathrm{S}^{p}, \Sigma, \mathbf{A}$ est isomorphe à l'un des trois systèmes primitifs a) $\sim(p+q+p), a z^{\sim}(3+q+3)$ ou $a z^{\sim}(3+q+2)$.

Enfin si $\Delta_{2} \neq \varnothing$, alors $\Sigma_{1}$ engendre un sous-système isomorphe à ay(p,p) (ou à $a z(3,3)$, ou à $a z(3,2))$, et le système engendré par $\Sigma_{2}$ est assez simple : il ne 
contient aucun élément projectif, ses composantes $\Delta$-connexes engendrent des soussystèmes isomorphes à $a a(q)$ et $a c^{*}(n)$ ( $n$ pair), et le diagramme de Dynkin de $\operatorname{supp}\left(\Sigma_{2}\right)$ est connexe. On voit facilement que $\Delta_{2}$ est distingué (non lisse), et que $\left(\mathrm{S}^{p}, \Sigma, \mathbf{A}\right) / \Delta_{2}$ est isomorphe à $a y^{\sim}(p+q+p)$ (ou à $a z^{\sim}(3+q+3)$, ou à $\left.a z^{\sim}(3+q+2)\right)$. Enfin, on vérifie sans peine que $\Delta_{1}, \Delta_{2}$ décompose $\mathrm{S}^{p}, \Sigma, \mathbf{A}$.

\subsection{Résultats complémentaires}

Il résulte de la preuve du théorème 1 que les sous-groupes magnifiques sont «presque tous» connexes.

Corollaire $\mathbf{1}$ - Soit $\mathrm{G}$ un groupe adjoint de type $\mathbf{A}$ et soit $\mathrm{H}$ un sous-groupe magnifique de G. On suppose $\mathrm{S}_{\mathrm{G} / \mathrm{H}}^{p}, \Sigma_{\mathrm{G} / \mathrm{H}}, \mathbf{A}_{\mathrm{G} / \mathrm{H}}$ cuspidal et irréductible. Alors $\mathrm{H}$ est connexe, sauf si $\mathrm{S}_{\mathrm{G} / \mathrm{H}}^{p}$, $\Sigma_{\mathrm{G} / \mathrm{H}}, \mathbf{A}_{\mathrm{G} / \mathrm{H}}$ est isomorphe :

ou bien à ao(n), $n$ impair $\geqslant 3$ (auquel cas $\mathrm{H}^{\circ}$ n'est pas magnifique);

ou bien à $a a^{*}(p+1+p), p \geqslant 1$, ou à ao(1) (auquel cas $\mathrm{H}^{\circ}$ est magnifique).

Gela résulte aussitôt de la preuve du théorème 1 .

Corollaire $\mathbf{2}-$ Soit $\mathrm{S}^{p}, \Sigma, \mathbf{A}$ un système sphérique adjoint de type $\mathbf{A}$, et soit $\Delta$ son ensemble des couleurs. Alors tout sous-ensemble distingué $\Delta^{\prime}$ de $\Delta$ possède la propriété $\left(^{*}\right)$ du $\S 3, n^{\circ} 3$.

Preuve - Montrons d'abord qu'on peut se ramener au cas où le système est cuspidal. Disons d'une couleur $\delta_{\alpha}$ de type $b$ qu'elle est parabolique, si $\alpha \notin \operatorname{supp} \Sigma$, et désignons par $\Delta_{p b}$ l'ensemble des couleurs paraboliques. Si $\Delta^{\prime}$ est un ensemble distingué de $\Delta$, alors $\Delta^{\prime} \backslash \Delta_{p b}$ est encore distingué dans $\Delta$. Par suite, le passage au quotient peut se faire en deux temps : d'abord par un $\Delta^{\prime}$ contenu dans $\Delta \backslash \Delta_{p b}$, puis par un $\Delta^{\prime}$ contenu dans $\Delta_{p b}$. Dans le deuxième cas le corollaire 2 est facile et, dans le premier, il suffit de montrer le corollaire 2 pour le système localisé en supp $\Sigma$, qui est cuspidal.

Lorsque le système est cuspidal, le passage au quotient se fait séparément dans chaque facteur irréductible, donc on peut supposer le système cuspidal irréductible. Tout système cuspidal et irréductible, qui contient une racine sphérique de type $a^{\prime}$, est isomorphe à $a a^{*}(p+1+q)(p \geqslant 1)$ ou à $a o(n)(n \geqslant 1)$. Dans ces cas, les seuls sous-ensembles distingués sont l'ensemble vide et l'ensemble $\Delta$.

Restent les systèmes $\mathrm{S}^{p}, \Sigma, \mathbf{A}$ cuspidaux, irréductibles, et ne contenant aucune racine sphérique de type $a^{\prime}$. D'après le théorème $1, \mathrm{~S}^{p}, \Sigma, \mathbf{A}$ est le triplet d'un $\mathrm{G} / \mathrm{H}$, où $\mathrm{H}$ est un sous-groupe magnifique de G. D'après 1.4 et la proposition $3.2, \Delta$ s'identifie à $\Delta_{\mathrm{G} / \mathrm{H}}$. D'après la théorie des plongements des espaces homogènes sphériques, au sous-espace vectoriel colorié $\mathrm{N}\left(\Delta^{\prime}\right), \Delta^{\prime}$ correspond un G-morphisme (à fibres connexes) $\mathrm{G} / \mathrm{H} \rightarrow \mathrm{G} / \mathrm{H}^{\prime}$. Par construction, le cône des valuations $\mathrm{V}_{\mathrm{G} / \mathrm{H}^{\prime}}$ est saillant (donc 
$\mathrm{N}_{\mathrm{G}}\left(\mathrm{H}^{\prime}\right) / \mathrm{H}^{\prime}$ est fini), et le triplet quotient s'identifie au système sphérique $\mathrm{S}_{\mathrm{G} / \mathrm{H}^{\prime}}^{p}, \Sigma_{\mathrm{G} / \mathrm{H}^{\prime}}$, $\mathbf{A}_{\mathrm{G} / \mathrm{H}^{\prime}}$. Reste à voir que $\Sigma_{\mathrm{G} / \mathrm{H}^{\prime}}$ engendre $\Xi_{\mathrm{G} / \mathrm{H}^{\prime}}$, autrement dit que $\mathrm{H}^{\prime}$ est magnifique.

Soit $\mathrm{H}^{*}$ le sous-groupe magnifique de $\mathrm{G}$ contenant $\mathrm{H}^{\prime}$ et dont le système sphérique est $\mathrm{S}_{\mathrm{G} / \mathrm{H}^{\prime}}^{p}, \Sigma_{\mathrm{G} / \mathrm{H}^{\prime}}, \mathbf{A}_{\mathrm{G} / \mathrm{H}^{\prime}}$. Puisque le triplet quotient d'un système sphérique ne contenant aucune racine sphérique de type $a^{\prime}$ ne contient également aucune racine de type $a^{\prime}, \mathrm{H}^{*}$ est connexe d'après le corollaire 1 . Par conséquent, $\mathrm{H}^{\prime}=\mathrm{H}^{*}$ est bien magnifique.

Soit $\mathrm{S}^{p}, \Sigma, \mathbf{A}$ un système sphérique adjoint de type $\mathrm{A}$, et soit $\Delta$ son ensemble des couleurs. Un couple $\Delta^{\prime}, \mathrm{S}^{\prime}$ (où $\Delta^{\prime} \subset \Delta$ et où $\mathrm{S}^{\prime} \subset \mathrm{S}$ ) sera dit distingué si $\Delta^{\prime}$ est distingué dans $\Delta$, si $\mathrm{S}^{\prime} \subset \mathrm{S} \cap \Sigma$, et si tout $\alpha \in \mathrm{S}^{\prime}$ vérifie $\Delta(\alpha) \cap \Delta^{\prime}=\varnothing$ et $\delta_{\alpha}^{+}(\gamma)=\delta_{\alpha}^{-}(\gamma)$, quel que soit $\gamma \in \Sigma$. Si $\phi: \mathrm{X} \rightarrow \mathrm{X}^{\prime}$ est un G-morphisme dominant entre G-variétés magnifiques, on pose $\Delta(\phi)=\left\{\mathrm{D} \in \Delta_{\mathrm{X}}, \phi(\mathrm{D})=\mathrm{X}^{\prime}\right\}$ et $\mathrm{S}(\phi)=\mathrm{S}_{\mathrm{X}}^{a} \cap \mathrm{S}_{\mathrm{X}^{\prime}}^{a^{\prime}}$.

Corollaire 3 - Soit $\mathrm{G}$ un groupe adjoint de type $\mathrm{A}$, et soit $\mathrm{X}$ une $\mathrm{G}$-variété magnifique. L'application qui à $\phi$ associe le couple $\Delta(\phi), \mathrm{S}(\phi)$ est une bijection entre l'ensemble des $\mathrm{G}$-morphismes dominants $\phi$ de $\mathrm{X}$ dans une autre $\mathrm{G}$-variété magnifique, et l'ensemble des couples distingués $\Delta^{\prime}, \mathrm{S}^{\prime}$ de $\mathrm{S}_{\mathrm{X}}^{p}, \Sigma_{\mathrm{X}}, \mathbf{A}_{\mathrm{X}}$.

La preuve du corollaire 3, proche de celle du corollaire 2, est laissée comme exercice.

Corollaire 4 - Soit $\mathrm{G}$ un groupe adjoint de type $\mathrm{A}$, et soit $\mathrm{H}$ un sous-groupe magnifique de G. Pour que $\mathrm{H}$ soit réductif, il faut et il suffit que le système sphérique de $\mathrm{G} / \mathrm{H}$ soit un produit de systèmes classiques et de systèmes $a c^{*}(n)$ (n pair), ax $(1+p+1,1)(p \geqslant 1)$, ax $(1,1,1)$ et ay $(p, p-1)$ $(p \geqslant 2)$.

Preuve - Soit $\mathrm{S}^{p}, \Sigma, \mathbf{A}$ le système sphérique de $\mathrm{G} / \mathrm{H}$, et soit $\rho: \Delta \rightarrow \mathrm{N} \supset \mathrm{V}$ son espace vectoriel colorié (qui s'identifie à l'espace vectoriel colorié de G/H). La réductivité de $\mathrm{H}$ équivaut aux conditions suivantes (voir ([Br6]) :

1) $0 \notin \rho(\Delta)$; et

2) il existe une forme linéaire sur $\mathrm{N}$ qui est $>0$ sur $\rho(\Delta)$ et $\leqslant 0$ sur $\mathrm{V}$.

De (1) et (2) résulte que $\mathrm{S}^{p}, \Sigma, \mathbf{A}$ est cuspidal, que $\mathbf{A}$ ne contient pas d'élément projectif, et que les composantes $\Delta$-connexes sont isolées. Par suite, $\mathrm{S}^{p}, \Sigma, \mathbf{A}$ est isomorphe à un produit de systèmes primitifs. Or les seuls systèmes primitifs qui vérifient (1) et (2) sont ceux mentionnés dans l'énoncé du corollaire 4. 


\section{§6 Clôtures sphériques}

Dans ce $\S$ et le suivant, sauf mention expresse du contraire, G désignera un groupe réductif connexe quelconque. On note $\mathrm{B}$ un sous-groupe de Borel de $\mathrm{G}$, et $\mathrm{T}$ un tore maximal de B.

Rappelons quelques faits bien connus (voir [Knl] ou [Br6]) qu'on va utiliser plusieurs fois. Soit $\mathrm{H}$ un sous-groupe sphérique de $\mathrm{G}$. Quitte à remplacer $\mathrm{H}$ par un sous-groupe conjugué, on peut (et on va) supposer que $\mathrm{BH}$ est ouvert dans G. Le groupe $\mathrm{N}_{\mathrm{G}}(\mathrm{H}) / \mathrm{H}$ est multiplicatif, et $\mathrm{BH}^{\circ}=\mathrm{BH}=\mathrm{BN}_{\mathrm{G}}\left(\mathrm{H}\right.$ ) (où $\mathrm{H}^{\circ}$ désigne la composante neutre de $\mathrm{H}$ ). Si $\mathrm{P}=\mathrm{P}_{\mathrm{G} / \mathrm{H}}$ est le sous-groupe parabolique associé à $\mathrm{G} / \mathrm{H}$ (voir §1), alors $\mathrm{BH} / \mathrm{H}$ est isomorphe à $\mathrm{P}^{u} \times \mathrm{Y}$, où $\mathrm{Y}$ est un espace homogène sous $\mathrm{T}$, dont l'algèbre affine est isomorphe à l'algèbre du groupe $\Xi_{\mathrm{G} / \mathrm{H}}$.

\subsection{La notion de clôture sphérique}

Le groupe des G-automorphismes de $\mathrm{G} / \mathrm{H}$ s'identifie à $\mathrm{N}_{\mathrm{G}}(\mathrm{H}) / \mathrm{H}$, donc $\mathrm{N}_{\mathrm{G}}(\mathrm{H})$ opère de façon naturelle dans $\Delta_{\mathrm{G} / \mathrm{H}}$. Notons $\overline{\mathrm{H}}$ le sous-groupe des éléments de $\mathrm{N}_{\mathrm{G}}(\mathrm{H})$ qui agissent trivialement dans $\Delta_{\mathrm{G} / \mathrm{H}}$. Le groupe $\overline{\mathrm{H}}$ est encore sphérique (dans $\mathrm{G}$ ) et le morphisme naturel $\mathrm{G} / \mathrm{H} \rightarrow \mathrm{G} / \overline{\mathrm{H}}$ induit une bijection $\Delta_{\mathrm{G} / \mathrm{H}} \rightarrow \Delta_{\mathrm{G} / \overline{\mathrm{H}}}$, permettant d'identifier ces deux ensembles.

On appellera $\overline{\mathrm{H}}$ la clôture sphérique de $\mathrm{H}$. Un sous-groupe $\mathrm{H}$ de $\mathrm{G}$ sera dit sphériquement clos s'il est sphérique et si $\overline{\mathrm{H}}=\mathrm{H}$.

La clôture sphérique de tout sous-groupe sphérique est sphériquement close. Par construction, le centre de $\mathrm{G}$ est contenu dans tout sous-groupe sphériquement clos $\mathrm{H}$, autrement dit $\mathrm{G} / \mathrm{H}$ est en fait un espace homogène du groupe adjoint de $\mathrm{G}$. L'intérêt de ces sous-groupes vient de ce que tout sous-groupe sphériquement clos est magnifique ([Kn2]).

L'objectif de ce $\S$ est une caractérisation combinatoire des sous-groupes sphériques ayant une clôture sphérique donnée (proposition 6.4).

\subsection{Sous-groupes sphériquement clos}

Soit $\mathrm{H}$ un sous-groupe sphériquement clos de G. Pour simplifier, on pose $\Xi=\Xi_{\mathrm{G} / \mathrm{H}}, \Sigma=\Sigma_{\mathrm{G} / \mathrm{H}}, \Delta=\Delta_{\mathrm{G} / \mathrm{H}}$ et $\rho=\rho_{\mathrm{G} / \mathrm{H}}$.

L'expression $\overline{\mathrm{H}^{\circ}}$ désigne bien sûr la clôture sphérique de $\mathrm{H}^{\circ}$. Posons $\Xi^{\circ}=\Xi_{\mathrm{G} / \mathrm{H}^{\circ}}$, $\Sigma^{\circ}=\Sigma_{\mathrm{G} / \overline{\mathrm{H}^{\circ}}}, \Delta^{\circ}=\Delta_{\mathrm{G} / \overline{\mathrm{H}^{\circ}}}, \rho^{\circ}=\rho_{\mathrm{G} / \overline{\mathrm{H}^{\circ}}}, \mathrm{S}^{\circ}=\mathrm{S} \cap \Sigma^{\circ} \cap \frac{1}{2} \Sigma$ et $m=\operatorname{card} \mathrm{S}^{\circ}$.

Lemme 6.2.1 - Le groupe $\mathrm{H} / \overline{\mathrm{H}^{\circ}}$ est isomorphe à $(\mathbf{Z} / 2 \mathbf{Z})^{m}$, et $\Xi^{\circ}$ est le sous-groupe de $\Xi(\mathrm{T})$ engendré par $\Xi$ et $\mathrm{S}^{\circ}$.

Preuve - Le morphisme naturel $\mathrm{G} / \overline{\mathrm{H}^{\circ}} \rightarrow \mathrm{G} / \mathrm{H}$ envoie les couleurs de $\Delta^{\circ}(\alpha)$ sur celles de $\Delta(\alpha)$, quel que soit $\alpha \in \mathrm{S}$. Par suite, l'opération naturelle de $\mathrm{H}$ dans $\Delta^{\circ}$ 
échange les deux éléments de $\Delta^{\circ}(\alpha)$ (pour chaque $\alpha \in \mathrm{S}^{\circ}$ ) et fixe les autres couleurs. D'où un morphisme injectif de groupes $\mathrm{H} / \overline{\mathrm{H}^{\circ}} \rightarrow(\mathrm{Z} / 2 \mathrm{Z})^{m}$. On en déduit l'inégalité card $\mathrm{H} / \overline{\mathrm{H}^{\circ}} \leqslant 2^{m}$.

Le nombre card $\mathrm{H} / \overline{\mathrm{H}^{\circ}}$ est aussi le degré de l'extension $\left[k\left(\mathrm{G} / \overline{\mathrm{H}^{\circ}}\right): k(\mathrm{G} / \mathrm{H})\right]$. Notons $\phi: \mathrm{BH} / \overline{\mathrm{H}^{\circ}} \rightarrow \mathrm{BH} / \mathrm{H}$ le morphisme naturel, et posons $\mathrm{Y}^{\circ}=\phi^{-1}(\mathrm{Y})$. Il est clair que le morphisme naturel $\mathrm{P}^{u} \times \mathrm{Y}^{\circ} \rightarrow \mathrm{BH} / \overline{\mathrm{H}^{\circ}}$ est un isomorphisme, d'où l'on déduit que $\mathrm{Y}^{\circ}$ est homogène sous $\mathrm{T}$ et que son algèbre affine est isomorphe à l'algèbre $\mathrm{du}$ groupe $\Xi^{\circ}$. Par suite, card $\mathrm{H} / \overline{\mathrm{H}}^{\circ}=\left[k\left(\mathrm{G} / \overline{\mathrm{H}}^{\circ}\right): k(\mathrm{G} / \mathrm{H})\right]=\left[k\left(\mathrm{Y}^{\circ}\right): k(\mathrm{Y})\right]=\operatorname{card} \Xi^{\circ} / \Xi$. Du fait que $\Xi^{\circ}$ et $\Xi$ sont des groupes libres de base $\Sigma^{\circ}$ et $\Sigma$, on déduit que $2^{m} \leqslant$ card $\Xi^{\circ} / \Xi$. Par conséquent, card $\mathrm{H} / \overline{\mathrm{H}}^{\circ}=\operatorname{card} \Xi^{\circ} / \Xi=2^{m}$. Les assertions du lemme en résultent.

Quitte à remplacer $G$ par un revêtement fini, on peut (et on va) supposer que $\mathrm{G}=\mathrm{C} \times \mathrm{G}_{1}$, où $\mathrm{C}$ est un tore et où $\mathrm{G}_{1}$ est un groupe semi-simple simplement connexe. Alors $k\left[\mathrm{G}_{1}\right]$ est une algèbre factorielle $([\mathrm{Iv}])$. On a $\mathrm{B}=\mathrm{C} \times \mathrm{B}_{1}$ où $\mathrm{B}_{1}$ est un sous-groupe de Borel de $\mathrm{G}$, et $\mathrm{H}=\mathrm{C} \times \mathrm{H}_{1}$, où $\mathrm{H}_{1}$ est un sous-groupe sphériquement clos de $\mathrm{G}_{1}$.

Désignons par ${ }^{(\mathrm{B})} k(\mathrm{G})^{(\mathrm{H})}$ le groupe multiplicatif des $f \in k(\mathrm{G})$ qui sont vecteurs propres pour l'opération de $\mathrm{B} \times \mathrm{H}$ dans $\mathrm{G}, \mathrm{B}$ opérant par «translations à gauche» et $\mathrm{H}$ par «translations à droite». Notons $\pi: \mathrm{G} \rightarrow \mathrm{G} / \mathrm{H}$ la projection naturelle. Pour tout $\mathrm{D} \in \Delta$, notons $f_{\mathrm{D}}$ l'équation de $\pi^{-1}(\mathrm{D})$ dans $k[\mathrm{G}]$ qui est constante égale à 1 sur $\mathrm{C}$. Pour tout $\chi \in \Xi(\mathrm{C})$, notons $f_{\chi}$ le caractère de $\mathrm{G}$ correspondant à $\chi$, modulo l'identification $\Xi(\mathrm{G})=\Xi(\mathrm{C})$ (pour l'opération de $\mathrm{G}$ dans $k(\mathrm{G})$ par «translations à gauche », $f_{\chi}$ est un vecteur propre de poids $\chi$ ).

\section{Lemme 6.2.2}

1) Les $f \in{ }^{(\mathrm{B})} k(\mathrm{G})^{(\mathrm{H})}$ sont ceux de la forme

$$
f=c f_{\chi} \prod_{\mathrm{D} \in \Delta}\left(f_{\mathrm{D}}\right)^{n(\mathrm{D})}\left(c \in k^{*}, \chi \in \Xi(\mathbf{G}) \text { et } n(\mathbf{D})_{\mathrm{D} \in \Delta} \in \mathbf{Z}^{\Delta}\right),
$$

et cette écriture est unique.

2) Les $\pi^{-1}(\mathrm{D})(\mathrm{D} \in \Delta)$ sont irréductibles, sauf si $\mathrm{D}=\mathrm{D}_{\alpha}^{\prime}$ avec $\alpha \in \mathrm{S}^{\circ}$, auquel cas $\pi^{-1}(\mathrm{D})$ possède deux composantes connexes qui sont échangées par $\mathrm{H}$ (opérant dans $\mathrm{G}$ par translations à droite).

Preuve. - Soit $f \in{ }^{(\mathrm{B})} k(\mathrm{G})^{\left(\mathrm{H}^{\circ}\right)}$. Puisque la restriction de $f$ à $\mathrm{C}$ est un vecteur propre de $\mathrm{C}$, il existe $c \in k^{*}$ et $\chi \in \Xi(\mathrm{C})$ tels que $f=c f_{\chi} g$, où $g \in k\left(\mathrm{G}_{1}\right)$ est un vecteur propre de $\mathrm{B}_{1} \times \mathrm{H}_{1}^{\circ}$ (valant 1 en l'élément neutre). Puisque $\mathrm{B}_{1} \times \mathrm{H}_{1}^{\circ}$ est connexe, tout facteur irréductible de $g$ est encore vecteur propre de $\mathrm{B}_{1} \times \mathrm{H}_{1}^{\circ}$. On peut identifier $\Delta^{\circ}$ à l'ensemble des composantes irréductibles de $\mathrm{G} \backslash \mathrm{BH}=\mathrm{G} \backslash \mathrm{BH}^{\circ}$. Pour tout $\mathrm{D} \in \Delta^{\circ}$, notons $f_{\mathrm{D}}$ l'équation de $\mathrm{D}$ dans $k\left[\mathrm{G}_{1}\right]$ qui vaut 1 en l'élément neutre. Tout élément irréductible de $k\left[\mathrm{G}_{1}\right]$ qui est vecteur propre de $\mathrm{B}_{1} \times \mathrm{H}_{1}^{\circ}$ est visiblement proportionnel 
à l'un des $f_{\mathrm{D}}, \mathrm{D} \in \Delta^{\circ}$. De la factorialité de $k\left[\mathrm{G}_{1}\right]$ résulte alors que

$$
(*) \quad f=c f_{\chi} \prod_{\mathrm{D} \in \Delta^{\circ}}\left(f_{\mathrm{D}}\right)^{n(\mathrm{D})}\left(c \in k^{*}, \chi \in \Xi(\mathrm{C}) \text { et } n(\mathrm{D})_{\mathrm{D} \in \Delta^{\circ}} \in \mathbf{Z}^{\Delta^{\circ}}\right)
$$

(et cette écriture est unique).

L'application qui à $\mathrm{D} \in \Delta$ associe $\pi^{-1}(\mathrm{D})$ permet d'identifier $\Delta$ à l'ensemble des orbites de $\mathrm{H}$ dans $\Delta^{\circ}$ (H opérant «par translations à droite»). Les assertions du lemme résultent de cette remarque, de $\left(^{*}\right)$ et du lemme 6.2.1.

\subsection{Un résultat intermédiaire}

On conserve les hypothèses et les notations précédentes.

Notons $\bar{\rho}: \Xi \rightarrow \Xi(\mathbf{C}) \times \mathbf{Z}^{\Delta}$ l'homomorphisme de groupe défini par $\bar{\rho}(\gamma)=\left(\left.\gamma\right|_{\Xi(\mathrm{C})}\right.$, $\left.<\rho(\mathrm{D}), \gamma>_{\mathrm{D} \in \Delta}\right), \gamma \in \Xi$.

Notons $\sigma: \Xi(\mathrm{C}) \times \mathbf{Z}^{\Delta} \rightarrow \Xi(\mathrm{B})$ et $\tau: \Xi(\mathrm{C}) \times \mathbf{Z}^{\Delta} \rightarrow \Xi(\mathrm{H})$ les homomorphismes de groupe définis par :

$$
\begin{aligned}
& \sigma\left(\left(\chi, n(\mathbf{D})_{\mathbf{D} \in \Delta}\right)\right)=\text { le poids de } \mathrm{B} \operatorname{de} f_{\chi} \prod_{\mathbf{D} \in \Delta}\left(f_{D}\right)^{n(\mathbf{D})} \text { et } \\
& \tau\left(\left(\chi, n(\mathbf{D})_{\mathbf{D} \in \Delta}\right)\right)=\text { le poids de } \mathrm{H} \operatorname{de} f_{\chi} \prod_{\mathbf{D} \in \Delta}\left(f_{\mathrm{D}}\right)^{n^{(\mathbf{D})}}, \quad\left(\chi, n(\mathbf{D})_{\mathbf{D} \in \Delta}\right) \in \Xi(\mathbf{C}) \times \mathbf{Z}^{\Delta} .
\end{aligned}
$$

Par définition de $\rho$ et de $\Xi$, l'application composée $\sigma \circ \bar{\rho}$ est l'identité de $\Xi$, et le noyau de $\tau$ est égal à l'image de $\bar{\rho}$.

Désignons par $\mathrm{H}^{*}$ l'intersection des noyaux des caractères de $\mathrm{H}\left(\mathrm{H} / \mathrm{H}^{*}\right.$ est le plus grand groupe quotient de $\mathrm{H}$ qui est un groupe multiplicatif). $\mathrm{Si} \mathrm{H}^{\prime}$ est un sous-groupe de $\mathrm{H}$ contenant $\mathrm{H}^{*}$, alors $\Xi\left(\mathrm{H} / \mathrm{H}^{\prime}\right)$ s'identifie à l'ensemble des caractères de $\mathrm{H}$ qui deviennent triviaux en restriction à $\mathrm{H}^{\prime}$. Posons $\Phi^{\prime}=\tau^{-1}\left(\Xi\left(\mathrm{H} / \mathrm{H}^{\prime}\right)\right)$ et $\Phi=\tau^{-1}(\{e\})=\bar{\rho}(\Xi)$.

Lemme 6.3.1 - L'application qui à $\mathrm{H}^{\prime}$ associe $\Phi^{\prime}$ est une bijection entre l'ensemble des sous-groupes $\mathrm{H}^{\prime}$ de $\mathrm{H}$ contenant $\mathrm{H}^{*}$, et l'ensemble des sous-groupes $\Phi^{\prime}$ de $\Xi(\mathrm{C}) \times \mathbf{Z}^{\Delta}$ contenant $\Phi$.

Preuve - Puisque $\mathrm{H} / \mathrm{H}^{*}$ est multiplicatif, l'application qui à $\mathrm{H}^{\prime}$ associe $\Xi\left(\mathrm{H}, \mathrm{H}^{\prime}\right)$ est une bijection entre l'ensemble des sous-groupes $\mathrm{H}^{\prime}$ de $\mathrm{H}$ contenant $\mathrm{H}^{*}$, et l'ensemble des sous-groupes de $\Xi(H)=\Xi\left(H / H^{*}\right)$. On a déjà mentionné que $\Phi=\tau^{-1}(\{e\})$. Pour établir le lemme, il reste à prouver que $\tau: \Xi(\mathrm{C}) \times \mathbf{Z}^{\Delta} \rightarrow \Xi(\mathrm{H})$ est surjectif.

Puisque le groupe $\mathrm{H} / \mathrm{H}^{*}$ opère fidèlement dans $\mathrm{G} / \mathrm{H}^{*}$, tout caractère de $\Xi(\mathrm{H})$ est poids d'un vecteur propre de $\mathrm{H}$ dans $k(\mathrm{G})$. Comme l'algèbre $k[\mathrm{G}]$ est factorielle, tout vecteur propre de $\mathrm{H}$ dans $k(\mathrm{G})$ est quotient de deux vecteurs propres de $\mathrm{H}$ dans $k[\mathrm{G}]$. Par suite, les poids de $\mathrm{H}$ dans $k[\mathrm{G}]^{\mathrm{H}^{*}}$ engendrent le groupe $\Xi(\mathrm{H})$. Puisque $k[\mathrm{G}]^{\mathrm{H}^{*}}$ est un G-module rationnel, tout poids de $\mathrm{H}$ dans $k[\mathrm{G}]^{\mathrm{H}^{*}}$ est poids d'un vecteur propre de $\mathrm{B} \times \mathrm{H}$ dans $k[\mathrm{G}]^{\mathrm{H}^{*}}$. La surjectivité de $\tau$ est alors conséquence du lemme 6.2.2. 
Lemme 6.3.2 - Soit $\mathrm{H}^{\prime}$ un sous-groupe de $\mathrm{H}$ contenant $\mathrm{H}^{*}$, et soit $\Phi^{\prime}$ le sous-groupe de $\Xi(\mathrm{C}) \times \mathbf{Z}^{\Delta}$ lui correspondant (d'après le lemme 6.3.1). Si $\mathrm{S}^{\circ} \cap \sigma\left(\Phi^{\prime}\right)=\varnothing$, alors $\mathrm{H}$ et $\mathrm{H}^{\prime}$ ont mêmes orbites dans l'ensemble des composantes irréductibles de $\mathrm{G} \backslash \mathrm{BH}$.

Preuve - Soit $\alpha \in \mathrm{S}^{\circ}$. Désignons par $\mathrm{H}_{\alpha}$ le sous-groupe des éléments de $\mathrm{H}$ qui fixent les deux composantes de $\pi^{-1}\left(\mathrm{D}_{\alpha}^{\prime}\right)$. Ce groupe est d'indice 2 dans $\mathrm{H}$, donc contient $\mathrm{H}^{*}$. Notons $\Phi_{\alpha}$ le sous-groupe de $\Xi(\mathrm{C}) \times \mathbf{Z}^{\Delta}$ qui lui correspond d'après le lemme précédent. Puisque card $\Delta_{\mathrm{G} / \mathrm{H}_{\alpha}}(\alpha)=2$, on a $\alpha \in \Xi_{\mathrm{G} / \mathrm{H}_{\alpha}}$; par suite, $\sigma\left(\Phi_{\alpha}\right)=\Xi_{\mathrm{G} / \mathrm{H}_{\alpha}}$ est le sous-groupe de $\Xi(\mathrm{B})$ engendré par $\Xi$ et $\alpha$. Si $\alpha \notin \sigma\left(\Phi^{\prime}\right)$, alors $\Phi^{\prime}$ ne contient pas $\Phi_{\alpha}$, ce qui implique que $H^{\prime}$ n'est pas contenu dans $H_{\alpha}$, ce qui démontre le lemme.

Lemme 6.3.3 - Soit $\mathrm{H}^{\prime}$ un sous-groupe de $\mathrm{H}$ contenant $\mathrm{H}^{*}$, et soit $\Phi^{\prime}$ le sous-groupe de $\Xi(\mathrm{C}) \times \mathbf{Z}^{\Delta}$ lui correspondant (d'après le lemme 6.3.1). Si la restriction de $\sigma$ à $\Phi^{\prime}$ est injective, alors $\mathrm{H}^{\prime}$ est un sous-groupe sphérique de $\mathrm{G}$. Si de plus $\mathrm{S}^{\circ} \cap \sigma\left(\Phi^{\prime}\right)=\varnothing$, alors la clôture sphérique de $\mathrm{H}^{\prime}$ est $\mathrm{H}$.

Preuve - Considérons les morphismes naturels

$$
\mathrm{BH} / \mathrm{H}^{*} \stackrel{\phi}{\longrightarrow} \mathrm{BH} / \mathrm{H}^{\prime} \stackrel{\psi}{\longrightarrow} \mathrm{BH} / \mathrm{H} \approx \mathrm{P}^{u} \times \mathrm{Y},
$$

et posons $\mathrm{Y}^{\prime}=\psi^{-1}(\mathrm{Y})$ et $\mathrm{Y}^{*}=\phi^{-1}\left(\mathrm{Y}^{\prime}\right)$. Il est clair que les applications naturelles $\mathrm{P}^{u} \times \mathrm{Y}^{\prime} \rightarrow \mathrm{BH} / \mathrm{H}^{\prime}$ et $\mathrm{P}^{u} \times \mathrm{Y}^{*} \rightarrow \mathrm{BH} / \mathrm{H}^{*}$ sont des isomorphismes, que $\mathrm{Y}^{\prime}$ (resp. $\mathrm{Y}^{*}$ ) est homogène sous $\mathrm{T} \times \mathrm{H} / \mathrm{H}^{\prime}$ (resp. $\mathrm{T} \times \mathrm{H} / \mathrm{H}^{*}$ ), et que les algèbres affines de $\mathrm{Y}^{\prime}$ et $\mathrm{Y}^{*}$ sont isomorphes aux algèbres de groupes $\Phi^{\prime}$ et $\Xi(\mathrm{C}) \times \mathbf{Z}^{\Delta}$. De l'injectivité de $\sigma$, il suit alors que le morphisme $\mathrm{B} \rightarrow \mathrm{BH} / \mathrm{H}^{\prime}$ est dominant, ce qui montre bien que $\mathrm{H}^{\prime}$ est sphérique.

Notons $\left(\mathrm{H}^{\circ}\right)^{*}$ l'intersection des noyaux des caractères de $\mathrm{H}^{\circ}$. Ce groupe n'a pas de caractères non triviaux et est connexe. De là et de $\mathrm{H}^{*} \subset \mathrm{H}^{\prime} \subset \mathrm{H}$ on déduit que $\left(\mathrm{H}^{\circ}\right)^{*} \subset\left(\mathrm{H}^{\prime}\right)^{\circ} \subset \mathrm{H}^{\circ}$.

Puisque $\left(\mathrm{H}^{\prime}\right)^{\circ}$ et $\mathrm{H}^{\circ}$ sont sphériques, pour toute représentation rationnelle irréductible $\mathrm{E}$ de $\mathrm{G}$, les vecteurs propres de $\left(\mathrm{H}^{\prime}\right)^{\circ}$ et de $\mathrm{H}^{\circ}$ forment une base de $\mathrm{E}^{\left(\mathrm{H}^{\circ}\right)^{*}}$, et tout vecteur propre de $\mathrm{H}^{\circ}$ est aussi vecteur propre de $\left(\mathrm{H}^{\prime}\right)^{\circ}$. Par conséquent, $\mathrm{H}^{\circ}$ et $\left(\mathrm{H}^{\prime}\right)^{\circ}$ ont mêmes vecteurs propres dans E. Puisque $k[\mathrm{G}]$, en tant que $\mathrm{G} \times \mathrm{G}$-module, est isomorphe à la somme directe des $\mathrm{E} \otimes \mathrm{E}^{*}$ ( $\mathrm{E}$ G-module rationnel irréductible), $\mathrm{B} \times\left(\mathrm{H}^{\prime}\right)^{\circ}$ et $\mathrm{B} \times \mathrm{H}^{\circ}$ ont aussi mêmes vecteurs propres dans $k[\mathrm{G}]$. Cela montre que $\mathrm{BH}^{\prime}=\mathrm{BH}$. Par hypothèse (et grâce au lemme précédent), $\mathrm{H}$ et $\mathrm{H}^{\prime}$ ont mêmes orbites dans l'ensemble des composantes irréductibles de $\mathrm{G} \backslash \mathrm{BH}$. On en déduit que les groupes $\mathrm{B} \times \mathrm{H}^{\prime}$ et $\mathrm{B} \times \mathrm{H}$ ont également mêmes vecteurs propres dans $k[\mathrm{G}]$. Comme $\mathrm{H}$ est sphériquement clos, cela montre bien que $\mathrm{H}$ est la clôture sphérique de $\mathrm{H}^{\prime}$.

Soit $\Xi^{\prime}$ un sous-groupe de $\Xi(\mathrm{B})$ contenant $\Sigma$, et soit $\rho^{\prime}: \Delta \rightarrow\left(\Xi^{\prime}\right)^{*}$ une application. Définissons, comme plus haut, $\overline{\rho^{\prime}}: \Xi^{\prime} \rightarrow \Xi(\mathrm{C}) \times \mathbf{Z}^{\Delta}$ par $\overline{\rho^{\prime}}(\gamma)=\left(\left.\gamma\right|_{\Xi(\mathrm{C})}, \quad\left\langle\rho^{\prime}(\mathrm{D}), \gamma>_{\mathrm{D} \in \Delta}\right)\right.$, $\gamma \in \Xi^{\prime}$. 
On dira que le couple $\Xi^{\prime}$, $\rho^{\prime}$ est adapté à $H$ s'il vérifie les trois conditions suivantes :

1) pour tout $\alpha \in \mathrm{S}^{\circ}$, on a $\alpha \notin \Xi^{\prime}$;

2) la restriction de $\overline{\rho^{\prime}}$ à $\Xi$ est égale à $\bar{\rho}$;

3) $\sigma \circ \overline{\rho^{\prime}}$ est l'identité de $\Xi^{\prime}$.

Proposition 6.3 - Soit $\mathrm{H}$ un sous-groupe sphériquement clos de G. L'application qui à un sous-groupe sphérique $\mathrm{H}^{\prime}$ associe le couple $\Xi_{\mathrm{G} / \mathrm{H}^{\prime}}, \rho_{\mathrm{G} / \mathrm{H}^{\prime}}$ est une bijection entre l'ensemble des sous-groupes sphériques $\mathrm{H}^{\prime}$ de $\mathrm{G}$ ayant $\mathrm{H}$ comme clôture sphérique, et l'ensemble des couples $\Xi^{\prime}, \rho^{\prime}$ adaptés à $\mathrm{H}$.

Preuve - Soit $\mathrm{H}^{\prime}$ un sous-groupe sphérique de $\mathrm{G}$ ayant $\mathrm{H}$ comme clôture sphérique. Le couple $\Xi_{\mathrm{G} / \mathrm{H}^{\prime}}, \rho_{\mathrm{G} / \mathrm{H}^{\prime}}$ vérifie les conditions (2) et (3) par construction. On sait que $\mathrm{H} / \mathrm{H}^{\prime}$ est un groupe multiplicatif (voir [Br6]), donc $\mathrm{H}^{*} \subset \mathrm{H}^{\prime}$. La condition (1) vient de ce que $\mathrm{H}^{\prime}$ permute les deux éléments de $\Delta_{\mathrm{G} / \mathrm{H}^{\prime}}(\alpha)$, quel que soit $\alpha \in \mathrm{S}^{\circ}$. L'application de la proposition est donc bien définie.

Puisque $\mathrm{H}^{*} \subset \mathrm{H}^{\prime}$, grâce au lemme 6.3.1, $\mathrm{H}^{\prime}$ est déterminé par $\Phi^{\prime}=\overline{\rho_{\mathrm{G} / \mathrm{H}^{\prime}}}\left(\Xi_{\mathrm{G} / \mathrm{H}^{\prime}}\right)$, donc est déterminé par le couple $\Xi_{\mathrm{G} / \mathrm{H}^{\prime}}, \rho_{\mathrm{G} / \mathrm{H}^{\prime}}$, ce qui montre que l'application de la proposition est injective.

Soit maintenant $\Xi^{\prime}, \rho^{\prime}$ un couple adapté à $H$. Posons $\Phi^{\prime}=\rho^{\prime}\left(\Xi^{\prime}\right)$. D'après la condition (2), $\Phi^{\prime}$ contient $\Phi$. D'après le lemme 6.3.1, $\Phi^{\prime}$ détermine donc un sousgroupe $\mathrm{H}^{\prime}$ de $\mathrm{H}$ contenant $\mathrm{H}^{*}$. D'après les conditions (1) et (3) et le lemme 6.3.3, $\mathrm{H}^{\prime}$ est sphérique dans $\mathrm{G}$, et sa clôture sphérique est $\mathrm{H}$. Enfin par construction on a $\Xi_{\mathrm{G} / \mathrm{H}^{\prime}}=\Xi^{\prime}$ et $\overline{\rho_{\mathrm{G} / \mathrm{H}^{\prime}}}=\left(\left.\sigma\right|_{\Phi^{\prime}}\right)^{-1}=\overline{\rho^{\prime}}$, donc $\rho_{\mathrm{G} / \mathrm{H}^{\prime}}=\rho^{\prime}$, ce qui termine la preuve de la proposition.

\subsection{Caractérisation combinatoire des sous-groupes sphériques ayant une clôture sphérique donnée}

Dans la suite on va reformuler les trois conditions (1), (2), (3) (disant qu'un couple $\Xi^{\prime}$, $\rho^{\prime}$ est adapté à $H$ ), de façon qu'elles ne dépendent plus que du système sphérique $\mathrm{S}^{p}, \Sigma, \mathbf{A}$ de $\mathrm{G} / \mathrm{H}$. Commençons par clarifier la définition de $\mathrm{S}^{\circ}$.

Rappelons que $\mathrm{S}^{a^{\prime}}=\mathrm{S} \cap \frac{1}{2} \Sigma$.

Lemme 6.4.1 - L'ensemble $\mathrm{S}^{\circ}$ est l'ensemble des $\alpha \in \mathrm{S}^{a^{\prime}}$ vérifiant $:\left\langle\beta^{\mathrm{v}}, \alpha\right\rangle$ est pair, quel que soit $\beta \in \mathrm{S}^{a^{\prime}}$.

Preuve - Soit $\alpha \in \mathrm{S}^{\circ}$. Alors $\alpha \in \Sigma^{\circ}$. Si $\beta \in \mathrm{S}^{a^{\prime}}$, alors :

ou bien $\beta \notin \mathrm{S}^{\circ}$, auquel cas $\mathrm{D}_{\beta}^{\prime} \in \Delta^{\circ}$ et $\left\langle\rho^{\circ}\left(\mathrm{D}_{\beta}^{\prime}\right), \alpha>=\frac{1}{2}<\beta^{\mathrm{v}}, \alpha>\in \mathbf{Z}\right.$, donc $<\beta^{\mathrm{v}}, \alpha>$ est pair; 
ou bien $\beta \in \mathrm{S}^{\circ}$, auquel cas $\Delta^{\circ}(\beta)=\left\{\mathrm{D}_{\beta}^{+}, \mathrm{D}_{\beta}^{-}\right\}$et $\left\langle\rho^{\circ}\left(\mathrm{D}_{\beta}^{+}\right), \alpha\right\rangle=\left\langle\rho^{\circ}\left(\mathrm{D}_{\beta}^{-}\right), \alpha\right\rangle$, donc $\left\langle\beta^{\mathrm{v}}, \alpha\right\rangle=2<\rho^{\circ}\left(\mathrm{D}_{\beta}^{+}\right), \alpha>$ est également pair.

Soit $\alpha \in \mathrm{S}^{a^{\prime}} \backslash \mathrm{S}^{\circ}$. Montrons qu'il existe $\beta \in \mathrm{S}^{a^{\prime}}$ tel que $<\beta^{\mathrm{v}}, \alpha>$ est impair. Raisonnons par l'absurde. Notons $\Xi^{\prime}$ le sous-groupe de $\Xi(\mathbf{B})$ engendré par $\Xi$ et $\alpha$. Si $<\beta^{\mathrm{v}}, \alpha>$ est pair, quel que soit $\beta \in \mathrm{S}^{a^{\prime}}$, alors le prolongement linéaire naturel de $\rho$ à $\Xi^{\prime}$ (notons le $\rho^{\prime}$ ) est à valeurs dans $\mathbf{Z}$, et le couple $\Xi^{\prime}, \rho^{\prime}$ est adapté à $H$. Soit $H^{\prime}$ le sous-groupe de $\mathrm{H}$ qui lui correspond d'après la proposition 6.3. Le groupe $\mathrm{H}^{\prime}$ est d'indice 2 dans $\mathrm{H}$, donc $\mathrm{H}^{\circ}=\left(\mathrm{H}^{\prime}\right)^{\circ}$. Comme $\alpha \in \Sigma_{\mathrm{G} / \mathrm{H}^{\prime}}$, il s'ensuit que $\alpha \in \Sigma^{\circ}$. Mais ce dernier signifie que $\alpha \in \mathrm{S}^{\circ}$, ce qui contredit l'hypothèse $\alpha \in \mathrm{S}^{a^{\prime}} \backslash \mathrm{S}^{\circ}$.

Rappelons qu'on a introduit au $\S 2, \mathrm{n}^{\circ} 3$, pour toute augmentation $\Xi^{\prime}, \rho^{\prime}$ d'un système sphérique $\mathrm{S}^{p}, \Sigma, \mathbf{A}$, un «ensemble de couleurs» $\Delta, \rho^{\prime}: \Delta \rightarrow\left(\Xi^{\prime}\right)^{*}$.

Lemme 6.4.2 - 1) Si $\Xi^{\prime}$, $\rho^{\prime}$ est un couple adapté à $\mathrm{H}$, alors $\Xi^{\prime}$, $\left.\rho^{\prime}\right|_{\mathrm{A}}$ est une augmentation de $\mathrm{S}^{p}, \Sigma, \mathbf{A}$.

2) Si $\Xi^{\prime}$, $\rho^{\prime}$ est une augmentation de $\mathrm{S}^{p}, \Sigma, \mathbf{A}$ et si $\Delta$, $\rho^{\prime}$ est son ensemble des couleurs, alors le couple $\Xi^{\prime}, \rho^{\prime}$ est adapté à $\mathrm{H}$.

Preuve - Pour tout $\mathrm{D} \in \Delta$, notons $\omega_{\mathrm{D}}$ le poids dans $\Xi(\mathrm{B})$ du B-vecteur propre $f_{\mathrm{D}}$. Si $n(\mathrm{D}, \boldsymbol{\alpha})$ sont les entiers tels que $\omega_{\mathrm{D}}=\sum_{\alpha \in \mathrm{S}} n(\mathrm{D}, \boldsymbol{\alpha}) \omega_{\alpha}$, alors on sait que

$$
\begin{aligned}
& n(\mathrm{D}, \alpha)=1 \text { si } \mathrm{D} \in \Delta(\alpha) \text { et } 2 \alpha \notin \Delta ; \\
& n(\mathrm{D}, \alpha)=2 \text { si } \mathrm{D} \in \Delta(\alpha) \text { et } 2 \alpha \in \Delta ; \\
& n(\mathrm{D}, \alpha)=0 \text { si } \mathrm{D} \notin \Delta(\alpha)
\end{aligned}
$$

(voir [Fo], p. 37-38).

Par suite, pour tout $\gamma \in \Xi^{\prime}$, on a $\left(\sigma \circ \overline{\rho^{\prime}}\right)(\gamma)=\left.\gamma\right|_{\Xi(\mathrm{C})}+\sum_{\mathrm{D} \in \Delta}\left\langle\rho^{\prime}(\mathrm{D}), \gamma>\omega_{\mathrm{D}}=\left.\gamma\right|_{\Xi(\mathrm{C})}+\right.$ $\sum_{\alpha \in \mathrm{S}} \sum_{\mathrm{D} \in \Delta(\alpha)}<n(\mathrm{D}, \alpha) \rho^{\prime}(\mathrm{D}), \gamma>\omega_{\alpha}$.

Par conséquent, (3) est équivalent à

$$
(*) \sum_{\mathrm{D} \in \Delta(\alpha)}<n(\mathrm{D}, \alpha) \rho^{\prime}(\mathrm{D}), \gamma>=<\alpha^{\mathrm{v}}, \gamma>\left(\alpha \in \mathrm{S} \text { et } \gamma \in \Xi^{\prime}\right) \text {. }
$$

Si $\alpha \in \mathrm{S}^{p},(s)$ équivaut à $(*)$, et si $\alpha \in \mathrm{S}^{a},(a 2)$ équivaut à $(*)$.

Si $\alpha \in \mathrm{S}^{a^{\prime}},(\sigma \mathrm{l})$ est équivalent à $(*)$ et $(1)$. En effet, $(\sigma \mathrm{l})$ assure que $\rho^{\prime}\left(\mathrm{D}_{\alpha}^{\prime}\right)$ est à valeurs entières sur $\Xi^{\prime}$, puis implique $\left(^{*}\right)$ et $(1)$; inversement, $\left(^{*}\right)$ implique que $\left\langle\alpha^{v}, \gamma\right\rangle$ est pair quel que soit $\gamma \in \Xi^{\prime}$, et (1) signifie $\alpha \notin \Xi^{\prime}$ si $\alpha \in S^{\circ}$; si $\alpha \notin S^{\circ}$, alors le lemme 6.4.1 montre qu'il existe $\beta \in \mathrm{S}^{a^{\prime}}$ tel que $\left\langle\beta^{\mathrm{v}}, \alpha\right\rangle$ est impair, ce qui implique encore $\alpha \notin \Xi^{\prime}$.

Si $\alpha \in \mathrm{S}^{b}$, (*) est vrai par construction de l'extension de $\rho^{\prime}$ à $\Delta$, grâce à $(\sigma 2)$; inversement $(\sigma 2)$ résulte de $(*)$ et de l'égalité $D_{\alpha}=D_{\beta}$ si $\alpha$ est orthogonal à $\beta$ et si $\alpha+\beta$ (ou $\left.\frac{1}{2}(\alpha+\beta)\right)$ appartient à $\Sigma$. 
Enfin, (2) équivaut à (al), ce qui termine la preuve du lemme 6.4.2.

On arrive au résultat principal de ce paragraphe.

Soit $\mathrm{H}$ un sous-groupe sphériquement clos de $\mathrm{G}$, et soit $\mathrm{S}^{p}$, $\Sigma$, A le système sphérique de $\mathrm{G} / \mathrm{H}$. Si $\mathrm{H}^{\prime}$ est un sous-groupe sphérique de $\mathrm{G}$ de clôture sphérique $\mathrm{H}$, alors $\mathbf{A}_{\mathrm{G} / \mathrm{H}^{\prime}}$ et $\mathbf{A}$ sont visiblement en bijection naturelle; on les identifiera dans ce qui suit.

Proposition 6.4 - L'application qui à $\mathrm{H}^{\prime}$ associe le couple $\Xi^{\prime}$, $\rho^{\prime}$ (où $\Xi^{\prime}=\Xi_{\mathrm{G} / \mathrm{H}^{\prime}}$ et où $\rho^{\prime}=$ la restriction de $\rho_{\mathrm{G} / \mathrm{H}^{\prime}}$ à $\left.\mathbf{A}\right)$, est une bijection entre l'ensemble des sous-groupes sphériques de $\mathrm{G}$ dont la clôture sphérique est $\mathrm{H}$, et l'ensemble des augmentations du système sphérique $\mathrm{S}^{p}, \Sigma, \mathbf{A}$.

Preuve - C'est une conséquence immédiate de la proposition 6.3 et du lemme 6.4.2.

\section{$\$ 7$ Preuve du théorème 2}

On conserve les notations et conventions du paragraphe précédent.

\subsection{Clôture sphérique d'un système sphérique}

Soit $\mathrm{S}^{p}, \Sigma, \mathbf{A}$ un système sphérique. On notera $2 \Sigma\left(\mathrm{S}^{p}\right)$ l'ensemble des $\gamma \in \Sigma \backslash \mathrm{S}$ vérifiant : $2 \gamma \in \Sigma(\mathrm{G})$ et le couple $2 \gamma, \mathrm{S}^{p}$ est celui d'une variété magnifique de rang 1 .

Pour tout $\gamma \in \Sigma$, on pose

$$
\bar{\gamma}=2 \gamma \text { si } \gamma \in 2 \Sigma\left(S^{p}\right) \text { et } \bar{\gamma}=\gamma \text { sinon. }
$$

On pose $\bar{\Sigma}=\{\bar{\gamma}, \gamma \in \Sigma\}$ et on notera $\bar{\Xi}$ le sous-groupe de $\Xi(\mathrm{B})$ engendré par $\bar{\Sigma}$. On désignera encore par A la famille de $\bar{\Xi}^{*}$ obtenue «par restriction» de A à $\bar{\Xi}$.

Il est clair que $\mathrm{S}^{p}, \bar{\Sigma}, \mathbf{A}$ est encore un système sphérique, qu'on appellera la clôture sphérique du système $\mathrm{S}^{p}, \Sigma, \mathbf{A}$. Un système sphérique qui est égal à sa clôture sphérique sera appelé sphériquement clos. Du fait que deux racines sphériques différentes et proportionnelles sont forcément l'une le double de l'autre (voir [Wa]), il résulte que la clôture sphérique d'un système sphérique est sphériquement close.

Soit $\mathrm{H}^{\prime}$ un sous-groupe sphérique de $\mathrm{G}$, et notons $\mathrm{H}$ la clôture sphérique de $\mathrm{H}^{\prime}$.

Lemme 7.1 - On suppose le théorème 1 vrai pour le groupe adjoint de G. Alors le système sphérique de $\mathrm{G} / \mathrm{H}$ est la clôture sphérique du système sphérique de $\mathrm{G} / \mathrm{H}^{\prime}$.

Preuve - Soit $\mathrm{S}^{p}, \Sigma$, A le système sphérique de $\mathrm{G} / \mathrm{H}$, et soit $\mathrm{S}^{p}, \bar{\Sigma}$, A la clôture sphérique de $\mathrm{S}^{p}, \Sigma, \mathbf{A}$. Si le théorème 1 est vrai pour le groupe adjoint de $\mathrm{G}$, alors il existe un sous-groupe magnifique $\overline{\mathrm{H}}$ ayant comme système sphérique $\mathrm{S}^{p}, \bar{\Sigma}$, A. Il est clair que $\Xi_{\mathrm{G} / \mathrm{H}}, \rho_{\mathrm{G} / \mathrm{H}}$ est une augmentation de $\mathrm{S}^{p}, \bar{\Sigma}, \mathbf{A}$. Soit $\mathrm{H}^{\diamond}$ le sous-groupe de $\overline{\mathrm{H}}$ 
correspondant à l'augmentation $\Xi_{\mathrm{G} / \mathrm{H}}, \rho_{\mathrm{G} / \mathrm{H}}$ de $\mathrm{S}^{p}, \bar{\Sigma}, \mathbf{A}$ (voir proposition 6.4). Alors $\mathrm{H}$ et $\mathrm{H}^{\diamond}$ sont deux sous-groupes magnifiques (contenant le centre de $\mathrm{G}$ ) dont les espaces homogènes $\mathrm{G} / \mathrm{H}$ et $\mathrm{G} / \mathrm{H}^{\diamond}$ ont même système sphérique. D'après l'assertion d'unicité du théorème $1, \mathrm{H}$ et $\mathrm{H}^{\diamond}$ sont conjugués. Puisque $\mathrm{H}$ est sphériquement clos et que la clôture sphérique de $\mathrm{H}^{\diamond}$ est $\overline{\mathrm{H}}$ (voir proposition 6.4), on a $\mathrm{H}^{\diamond}=\overline{\mathrm{H}}$, ce qui démontre le lemme.

Remarque - Lorsque $\mathrm{G}$ est adjoint de type $\mathrm{A}$, tout système sphérique est «sphériquement clos». En effet, pour tout $\gamma \in \Sigma(G) \backslash S$ on a alors toujours $2 \gamma \notin \Sigma(G)$.

\subsection{Preuve du théorème 2}

1) Montrons que l'application du théorème est bien définie. Soit $\mathrm{H}^{\prime}$ un sousgroupe sphérique de G. Il faut expliquer pourquoi le quintuplet $\mathrm{S}_{\mathrm{G} / \mathrm{H}^{\prime}}^{p}, \Sigma_{\mathrm{G} / \mathrm{H}^{\prime}}, \mathbf{A}_{\mathrm{G} / \mathrm{H}^{\prime}}$, $\Xi_{\mathrm{G} / \mathrm{H}^{\prime}}, \rho_{\mathrm{G} / \mathrm{H}^{\prime}}$ est une donnée sphérique homogène.

Commençons par expliciter pourquoi $\mathrm{S}_{\mathrm{G} / \mathrm{H}^{\prime}}^{p}, \Sigma_{\mathrm{G} / \mathrm{H}^{\prime}}, \mathbf{A}_{\mathrm{G} / \mathrm{H}^{\prime}}$ est un système sphérique. Soit $\mathrm{H}$ la clôture sphérique de $\mathrm{H}^{\prime}$ et soit $\mathrm{S}^{p}, \Sigma, \mathbf{A}$ le système sphérique de $\mathrm{G} / \mathrm{H}$. Comme $\Delta_{\mathrm{G} / \mathrm{H}^{\prime}}(\alpha) \rightarrow \Delta(\alpha)$ est bijectif, quel que soit $\alpha \in \mathrm{S}$, on déduit que $\mathrm{S}_{\mathrm{G} / \mathrm{H}^{\prime}}^{p}=\mathrm{S}^{p}$, que $\mathrm{S} \cap \Sigma_{\mathrm{G} / \mathrm{H}^{\prime}}=\mathrm{S} \cap \Sigma$, que $\mathrm{S} \cap \frac{1}{2} \Sigma_{\mathrm{G} / \mathrm{H}^{\prime}}=\mathrm{S} \cap \frac{1}{2} \Sigma$ et que $\mathbf{A}_{\mathrm{G} / \mathrm{H}^{\prime}}$ peut être identifié à $\mathrm{A}$ (en tant qu'ensemble). Puisque $\Sigma_{\mathrm{G} / \mathrm{H}^{\prime}}$ et $\Sigma$ définissent tous deux le cône simplicial $\mathrm{V}_{\mathrm{G} / \mathrm{H}^{\prime}}$ dans $\mathrm{N}_{\mathrm{G} / \mathrm{H}^{\prime}}$, pour tout $\gamma \in \Sigma_{\mathrm{G} / \mathrm{H}^{\prime}}$, on a ou bien $\gamma \in \Sigma$, ou bien $2 \gamma \in \Sigma$ (deux racines sphériques différentes et proportionnelles sont l'une le double de l'autre, voir [Wa]). De cela et du fait (vu au §6) que $\mathrm{S}^{p}, \Sigma, \mathbf{A}, \Xi_{\mathrm{G} / \mathrm{H}^{\prime}}, \rho_{\mathrm{G} / \mathrm{H}^{\prime}}$ est un système sphérique augmenté, on déduit sans peine que $\mathbf{A}_{\mathrm{G} / \mathrm{H}^{\prime}}$ est adapté à $\Sigma_{\mathrm{G} / \mathrm{H}^{\prime}}$ et que $\mathrm{S}_{\mathrm{G} / \mathrm{H}^{\prime}}^{p}, \Sigma_{\mathrm{G} / \mathrm{H}^{\prime}}, \mathbf{A}_{\mathrm{G} / \mathrm{H}^{\prime}}$ vérifie $(\Sigma 1)$ et $(\Sigma 2)$. Par définition, tout $\gamma \in \Sigma_{\mathrm{G} / \mathrm{H}^{\prime}}$ peut être réalisé comme racine sphérique d'une sous-variété magnifique $\mathrm{X}$ de rang 1 d'un plongement de $\mathrm{G} / \mathrm{H}^{\prime}$; avec un peu de soin, on peut choisir l'éventail colorié de ce plongement «sans couleurs», auquel cas il est bien connu que $\mathrm{S}_{\mathrm{X}}^{p}=\mathrm{S}_{\mathrm{G} / \mathrm{H}^{\prime}}^{p}$, ce qui montre que $\mathrm{S}_{\mathrm{G} / \mathrm{H}^{\prime}}^{p}$, $\Sigma_{\mathrm{G} / \mathrm{H}^{\prime}}, \mathbf{A}_{\mathrm{G} / \mathrm{H}^{\prime}}$ vérifie aussi la condition $(\mathrm{S})$.

Du fait que $\Xi_{\mathrm{G} / \mathrm{H}^{\prime}}, \rho_{\mathrm{G} / \mathrm{H}^{\prime}}$ est une augmentation de $\mathrm{S}^{p}, \Sigma, \mathbf{A}$, on déduit sans peine que $\Xi_{\mathrm{G} / \mathrm{H}^{\prime}}, \rho_{\mathrm{G} / \mathrm{H}^{\prime}}$ est aussi une augmentation de $\mathrm{S}_{\mathrm{G} / \mathrm{H}^{\prime}}^{p}, \Sigma_{\mathrm{G} / \mathrm{H}^{\prime}}, \mathbf{A}_{\mathrm{G} / \mathrm{H}^{\prime}}$. Enfin, les éléments de $\Sigma_{\mathrm{G} / \mathrm{H}^{\prime}}$ sont par définition primitifs dans $\Xi_{\mathrm{G} / \mathrm{H}^{\prime}}$.

2) Montrons maintenant que l'application du théorème 2 est injective. Notons $\mathrm{H}$ la clôture sphérique de $\mathrm{H}^{\prime}$. D'après le lemme 7.1 , le système sphérique de $\mathrm{G} / \mathrm{H}^{\prime}$ détermine celui de $\mathrm{G} / \mathrm{H}$ (noté $\mathrm{S}^{p}, \Sigma, \mathbf{A}$ ). D'après le théorème 1 , ce dernier détermine $H$ (à conjugaison près). Mais $\Xi_{\mathrm{G} / \mathrm{H}^{\prime}}, \rho_{\mathrm{G} / \mathrm{H}^{\prime}}$ est une augmentation de $\mathrm{S}^{p}, \Sigma$, A qui, d'après la proposition 6.4, détermine $\mathrm{H}^{\prime}$. En résumé, $\mathrm{H}^{\prime}$ est bien déterminé (à conjugaison près) par la donnée sphérique homogène de $\mathrm{G} / \mathrm{H}^{\prime}$.

3) Enfin, montrons que l'application du théorème 2 est aussi surjective. Soit $\mathrm{S}^{p}$, $\Sigma, \mathbf{A}, \Xi^{\prime}, \rho^{\prime}$ une donnée sphérique homogène et soit $S^{p}, \bar{\Sigma}, \mathbf{A}$ la clôture sphérique de $S^{p}$, 
$\Sigma$, A. D'après le théorème 1 et le lemme 7.1 , il existe un sous-groupe sphériquement clos $\mathrm{H}$ dont l'espace homogène $\mathrm{G} / \mathrm{H}$ possède $\mathrm{S}^{p}, \bar{\Sigma}, \mathbf{A}$ comme système sphérique. Le couple $\Xi^{\prime}, \rho^{\prime}$ est aussi une augmentation de $\mathrm{S}^{p}, \bar{\Sigma}$, A. Soit $\mathrm{H}^{\prime}$ le sous-groupe de $\mathrm{H}$ qui lui correspond d'après la proposition 6.4. Alors par construction $\mathrm{G} / \mathrm{H}^{\prime}$ possède $\mathrm{S}^{p}, \Sigma, \mathbf{A}, \Xi^{\prime}, \rho^{\prime}$ comme donnée sphérique homogène, ce qui termine la preuve du théorème 2 .

\section{BIBLIOGRAPHIE}

[Ak] D. N. AkHiEzer, Equivariant completion of homogeneous algebraic varieties by homogeneous divisors, Ann. Global Anal. Geom., 1 (1983), 49-78.

[Brl] M. Brion, On spherical varieties of rank one, CMS Conf. Proc., 10 (1989), 31-41.

[Br2] M. Brion, Classification des espaces homogènes sphériques, Compositio Math., 63 (1987), 189-208.

[B3] M. Brion, Sur l'image de l'application moment, Lect. Notes in Math., Springer, 1296 (1987), 177-192.

[Br4] M. Brion, Sur la géométrie des variétés sphériques, Comment. Math. Helv., 66 (1991), 237-262.

[Br5] M. BRion, Spherical varieties, Proc. ICM 1994, vol. 2, Birkhäuser (1995), 753-760.

[Br6] M. BRION, Variétés sphériques, www.fourier.ujf-grenoble.fr/ mbrion/notes.html

[Br7] M. BRION, On orbit closures of Borel subgroups in spherical varieties, www.fourier.ujf.-grenoble.fr/ mbrion/preprints.html

[DeG-P] G. De Concini, G. Procesi, Complete Symmetric Varieties, Lect. Notes in Math., Springer, 996 (1983), $1-44$.

[De] M. Demazure, Sous-groupes algébriques de rang maximum du groupe de Cremona, Ann. Sci. de l'École Norm. Sup., 4 érie, t. 3 (1970), 507-588.

[Fo] A. Foschi, Variétés magnifiques et polytopes moment, thèse de doctorat de l'Univ. de Grenoble I, 1998.

[Fu] W. Fulton, Introduction to Toric Varieties, Ann. of Math. Studies, 131, Princeton Univ. Press, 1993.

[G-S] V. Gulllemin, S. Sternberg, Multiplicity-free spaces, f. Diff. Geom., 19 (1984), 31-56.

[Ho] R. Howe, Perspectives on invariant theory : Schur duality, multiplicity-free actions and beyond, Israel Math. Conf. Proc., vol. 8, 1995.

[H-W] A. Huckleberry, T. Wurzbacher, Multiplicity-free complex manifolds, Math. Ann., 286 (1990), 261-280.

[Iv] B. Iversen, The geometry of algebraic groups, Advances in Math., 20 (1976), 57-85.

[Kn1] F. Knop, The Luna-Vust theory of spherical embeddings, Proc. of the Hyderabad conference on algebraic groups (1989), Manj-Prakashan (1991), 225-249.

[Kn2] F. Knop, Automorphisms, root systems and the compactification of homogeneous varieties, f. Amer. Math. Soc. 1, vol. 9 (1996), 153-174.

[Kn3] F. KNoP, Weyl groups of hamiltonian manifolds I, www.math.rutgers.edu/ knop/abstracts/abstracts.html

[Kn4] F. KNop, Towards a classification of multiplicity free manifolds, texte d'un exposé au colloque S.M.F. «Opérations hamiltoniennes et opérations de groupes algébriques », Grenoble, 1997, à paraître.

[Kn5] F. Knop, On the set of orbits for a Borel subgroup, Comment. Math. Helv., 70 (1995), 285-309.

[Lul] D. Luna, Toute variété magnifique est sphérique, Trans. Groups, vol. 1, nº 3 (1996), 249-258.

[Lu2] D. Luna, Grosses cellules pour les variétés sphériques, in Algebraic Groups and Lie Groups, ed. by G. I. Lehrer, Australian Math. Soc. Lecture, Series 9 (1997), 267-280.

[LV] D. Luna, Th. Vust, Plongements d'espaces homogènes, Comment. Math. Helvetici, 58 (1983), 186-245.

[Mi] I. Mıкітуик, On the integrability of invariant hamiltonian systems with homogeneous configuration spaces, Math. USSR, Sb 57 (1987), 527-546.

[Vul] Th. Vust, Sur la théorie des invariants des groupes classiques, Ann. Inst. Fourier, 26 (1976), 1-31. 
[Vu2] Th. Vust, Plongements d'espaces symétriques algébriques : une classification, Ann. Sc. Norm. Sup. Pisa, Série IV, vol. XVII, fasc. 2 (1990), 165-194.

[Wa] B. Wasserman, Wonderful varieties of rank two, Trans. Groups, vol. 1, n ${ }^{\circ} 4$ (1996), 375-403.

[Wo] G. Woodward, Spherical varieties and existence of invariant Kähler structures, Duke Math. F., vol. 93, $\mathrm{n}^{\circ} 2$ (1998), 345-377.

D. L.

Institut Fourier

Université de Grenoble I

BP 74

38402 Saint-Martin-d'Hères, France

Manuscrit reçu le 13 juin 2000. 